\title{
Why do only some women develop postmenopausal osteoporosis?
}

by

Julia Maria Pasquale

A thesis submitted in conformity with the requirements for the degree of Master of Science

Department of Laboratory Medicine and Pathobiology

University of Toronto

(C) Copyright by Julia Maria Pasquale (2016) 


\title{
Why do only some women develop postmenopausal osteoporosis?
}

\author{
Julia Maria Pasquale \\ Master of Science \\ Laboratory Medicine and Pathobiology \\ University of Toronto \\ 2016
}

\begin{abstract}
Although all women experience postmenopausal bone loss, only $25-30 \%$ will develop postmenopausal osteoporosis, therefore another mechanism must be working with estrogen deficiency to cause this disease. Our proposed mechanism outlines the accumulation of advanced glycation endproducts (AGEs) as the driving force behind bone loss through intracellular signaling after ligation to the receptor for advanced glycation endproducts (RAGE). The study employed a high-fructose diet to initiate production of AGEs and investigated the effects on bone loss and RAGE expression after ovariectomy. This study investigated changes in bone quality and oxidative damage due to diet and surgeries as well as levels of soluble and bone collagen bound AGEs. It also worked to evaluate RAGE expression in serum and osteoclasts. Results show there was no production of AGEs, and bone quality and oxidative damage were affected by ovariectomy only. Soluble RAGE expression was increased due to ovariectomy alone, however cell-bound RAGE was unchanged.
\end{abstract}




\section{ACKNOWLEDGEMENTS}

Firstly, I would like to express my sincerest gratitude to my supervisors Dr. Marc Grynpas and Dr. Thomas Willett, who have both provided me with continuous support throughout my studies. Thank you for your patience, motivation and knowledge; your guidance has greatly helped me in the completion of my project. To my graduate committee members Dr. Rita Kandel and Dr. Michael Glogauer, thank you for your insightful comments and encouragements.

I would also like to thank several members of the Grynpas lab. To Jindra Tupy, thank you for your contributions to this project, your technical expertise as well as your friendship was instrumental in the completion of this study. To Sally Hu, you have given me exceptional support both as a mentor and as a friend, I am eternally grateful to have shared this experience with you. To Ariana dela Cruz, Lucia Zhang, Elise Post, Tanya Raaphorst, Adeline $\mathrm{Ng}$, and Yaryna Storozhuk, thank you for providing me with an encouraging and exciting work environment. I would never have made it through without your assistance, training, and answering of endless questions. To Sam Park, Mitchell Woodside and Justin Parreno, thank you for the never-ending conversations and laughter, you each truly helped to create a wonderful, fun and memorable work environment. Finally, thank you to Richard Cheung, Tarik Attia, Seanna Yoon and Bedilu Allo, each of you greatly contributed to my time spent in the lab.

I would also like to acknowledge Dr. Kate Banks, Frank Guiliano, Jenn Martin and other veterinarian technicians who assisted in my study. Your technical expertise and compassion for animals was both inspiring and invaluable.

Finally, I would like to thank my family and friends for your endless support and love throughout my studies. To my mom, I would have never been able to accomplish what I have if it wasn't for your constant encouragement. Your love and inspiration means more to me then you will ever know. Thank you for all that you do. 


\section{TABLE OF CONTENTS}

LIST OF FIGURES ................................................................................................ vii

LIST OF TABLES ...........................................................................................................

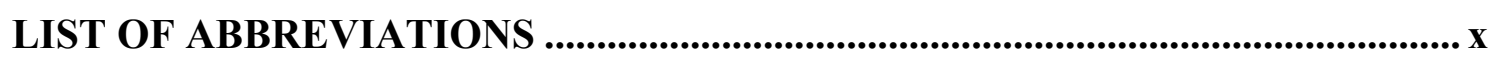

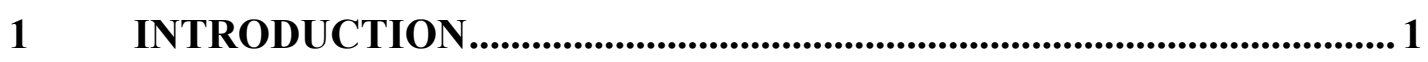

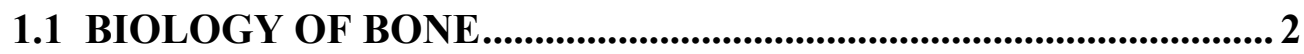

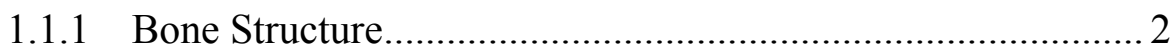

1.1.2 Bone Composition............................................................... 4

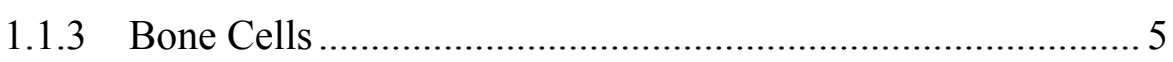

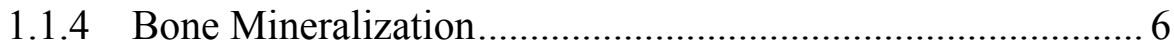

1.1.5 Bone Remodeling........................................................... 7

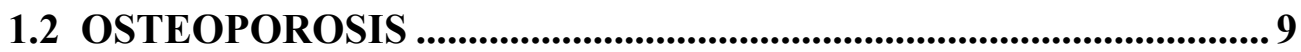

1.2.1 Postmenopausal Osteoporosis ................................................ 11

1.3 NEW THEORY OF POSTMENOPAUSAL OSTEOPOROSIS ..... 12

1.3.1 Advanced Glycation Endproducts .......................................... 15

1.3.2 Receptor for Advanced Glycation Endproducts ................... 18

1.4 AGE-RAGE INTERACTION ............................................................. 19

1.4.1 AGE-RAGE and Osteoclasts ................................................ 20

1.4.2 AGE-RAGE and Osteoblasts ................................................. 21

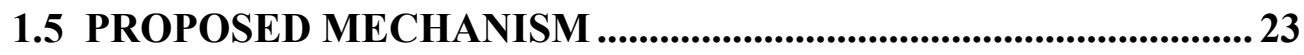

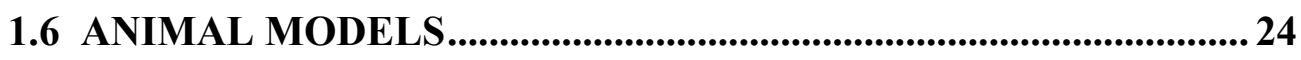

1.6.1 Rat Ovariectomy Model........................................................ 24

1.6.2 High Fructose Diet Model...................................................... 25

1.7 BONE QUALITY ................................................................................... 26

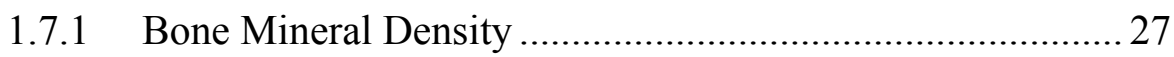

1.7.2 Geometry and Microarchitecture ........................................... 28

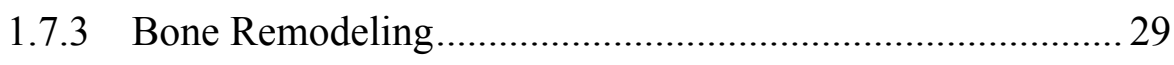

1.8 BLOOD AND BONE BIOCHEMISTRY ......................................... 29

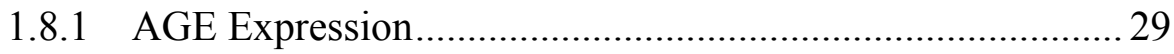

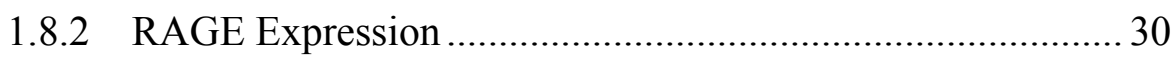


1.8.3 Oxidative Stress Expression.................................................. 30

1.8.4 Enzymatic Collagen Crosslink Expression .............................. 31

2 STUDY OBJECTIVES AND HYPOTHESIS ....................................... 32

3 MATERIALS AND METHODS .............................................................. 33

3.1 ANIMAL HUSBANDRY .....................................................................33

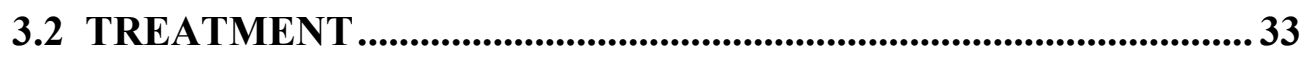

3.2.1 Sacrifice and Dissection ........................................................... 36

3.3 EVALUATION OF TREATMENT EFFECTS ....................................36

3.3.1 Dual-Energy X-Ray Absorptiometry (DXA) ........................ 38

3.3.2 X-Ray Microcomputed Tomography (microCT) .................. 38

3.3.3 Undecalcified Histomorphometry ........................................... 41

3.3.4 Decalcified Histomorphometry .............................................. 45

3.3.5 Hematoxylin and Eosin Staining .......................................... 47

3.3.6 Enzyme-Linked Immunosorbent Assay (ELISA) ................. 47

3.3.7 Protein Carbonylation Assay ................................................ 48

3.3.8 High-Performance Liquid Chromatography ......................... 49

3.3.9 Immunohistochemistry ..................................................... 50

3.6 STATISTICAL ANALYSIS .............................................................. 52

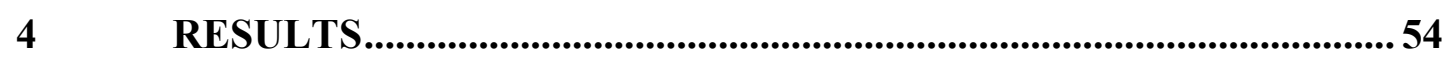

4.1 ANIMAL WEIGHTS..................................................................... 54

4.2 DXA ANALYSIS ...........................................................................54

4.3 MICROCT ANALYSIS.................................................................57

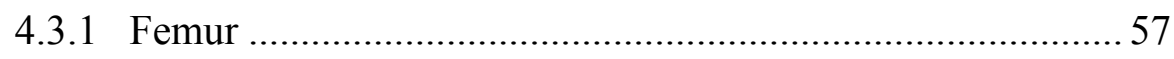

4.3.2 L6 Vertebrae .................................................................. 59

4.4 UNDECALCIFIED HISTOMORPHOMETRY ........................... 62

4.4.1 Goldner's Trichrome Stain....................................................... 63

4.4.2 Calcein Green Labeling........................................................... 65

4.5 DECALCIFIED HISTOMORPHOMETRY ..................................68

4.6 HEMATOXYLIN AND EOSIN STAINING ...................................69

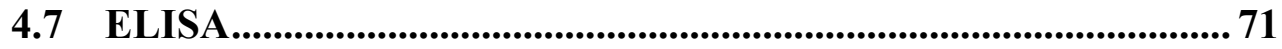

4.7.1 Soluble Pentosidine ............................................................... 71 


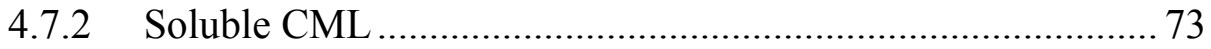

4.7.3 Soluble RAGE .................................................................. 75

4.8 PROTEIN CARBONYLATION ASSAY ......................................... 77

4.9 HPLC ......................................................................................................... 78

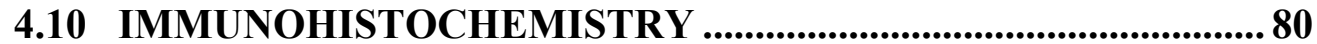

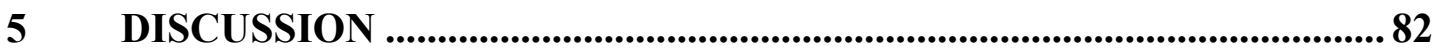

5.1 STUDY OBJECTIVES ......................................................................... 82

5.2 OBJECTIVE 1........................................................................................ 83

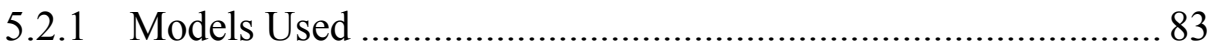

5.2.2 Gross Effects of Diets and Surgeries....................................... 84

5.2.3 Diet Effects on AGE Production ............................................. 85

5.2.4 Diet and Surgery Effects on AGE Production ......................... 87

5.2.5 Diet Effects on Bone Quality ................................................... 89

5.2.6 Diet and Surgery Effects on Bone Quality................................ 90

5.2.7 Objective 1: Concluding Summary …………………………... 95

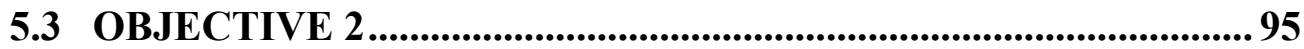

5.3.1 Oxidative Damage.................................................................... 96

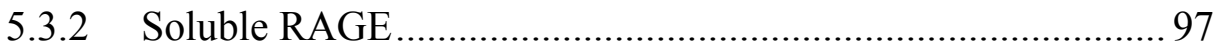

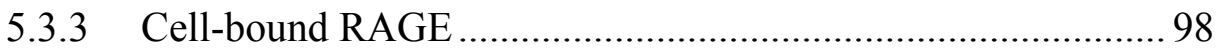

5.3.4 Objective 2: Concluding Summary ......................................... 100

5.4 OVERALL CONCLUSIONS............................................................ 100

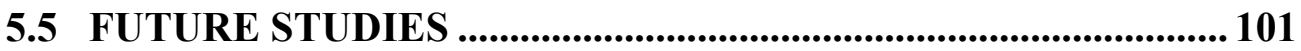

REFERENCES................................................................................................................... 104 


\section{LIST OF FIGURES}

Figure 1-1: Illustration or cortical and cancellous bone.......................................... 2

Figure 1-2: Illustration of different regions of the long bone ................................ 3

Figure 1-3: Illustration of the hierarchical structure of bone ................................... 4

Figure 1-4: Illustration of the bone remodeling cycle ............................................. 9

Figure 1-5: MicroCT images of osteoporotic and normal trabecular bone............... 11

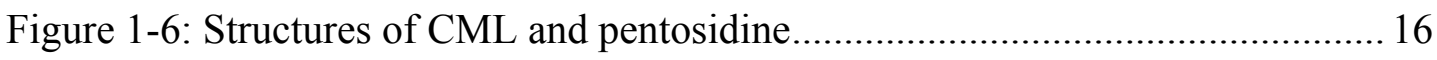

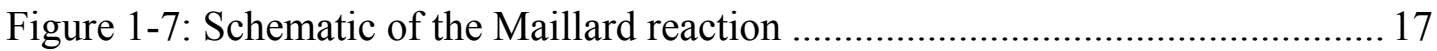

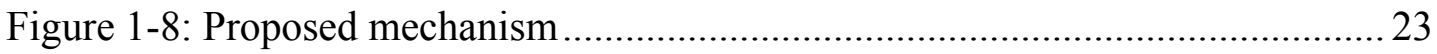

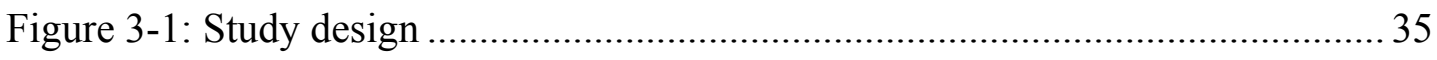

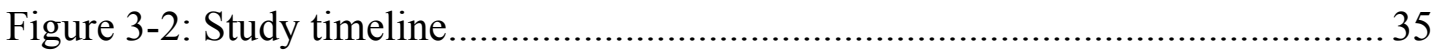

Figure 3-3: Representative images of microCT scanning view .............................. 39

Figure 3-4: Representative images of cortical bone ROIs for microCT .................. 40

Figure 3-5: Representative images of trabecular bone ROIs for microCT ............... 41

Figure 3-6: Representative image of Goldner's Trichrome stain............................ 42

Figure 3-7: ROI for all histological analysis of trabecular bone ............................ 43

Figure 3-8: Representative image of calcein green labeling ................................. 44

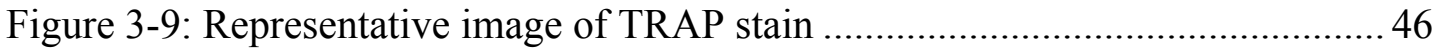

Figure 3-10: Representative images of TRAP and immunohistochemistry ..............52

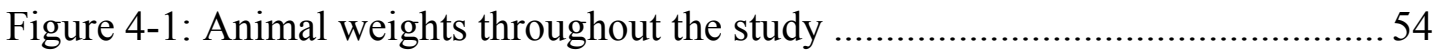

Figure 4-2: DXA results for femurs and L6s of baseline groups ........................... 55

Figure 4-3: DXA results for femurs of surgical groups ..................................... 56

Figure 4-4: DXA results for L6 vertebrae of surgical groups ................................ 57

Figure 4-5: Representative microCT cross-sectional images of femurs ...................58

Figure 4-6: vBMD of cortical bone from microCT analysis................................59

Figure 4-7: Representative microCT cross-sectional image of L6 vertebrae............. 60

Figure 4-8: MicroCT results for L6 vertebrae of surgical groups ........................... 62

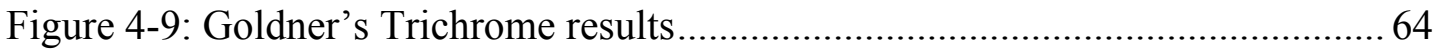

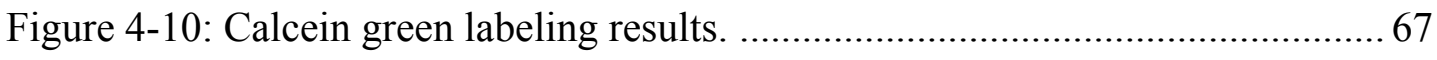

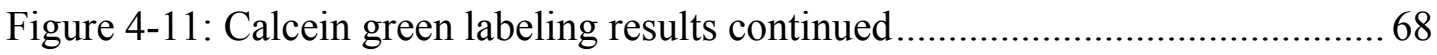

Figure 4-12: Representative images of livers from baseline groups ....................... 70 
Figure 4-13: Representative images of livers from surgical groups ....................... 70

Figure 4-14: ELISA results for pentosidine at each time-point for surgical groups .. 72

Figure 4-15: ELISA results for CML one month after surgeries ............................ 74

Figure 4-16: ELISA results for CML at each time-point for surgical groups ............ 74

Figure 4-17: ELISA results for sRAGE one month after surgeries ........................ 76

Figure 4-18: ELISA results for sRAGE at each time-point for surgical groups ........ 76

Figure 4-19: Carbonylation results for insoluble collagen fraction ......................... 78

Figure 4-20: HPLC results for bone collagen deoxypyridinoline ............................ 80

Figure 4-21: Representative images of immunohistochemistry.............................. 81 


\section{LIST OF TABLES}

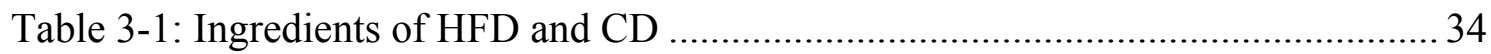

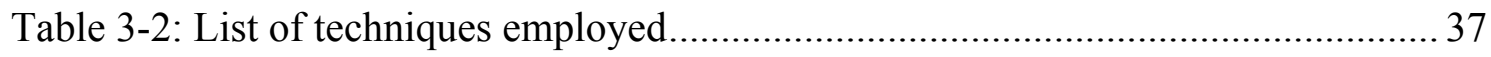

Table 4-1: MicroCT results of femurs from baseline groups............................................ 58

Table 4-2: MicroCT results of femurs from surgical groups .......................................... 59

Table 4-3: MicroCT results of L6 vertebrae from baseline groups .................................. 61

Table 4-4: MicroCT results of L6 vertebrae from surgical groups.................................. 61

Table 4-5: Goldner's Trichrome results from baseline groups ........................................ 63

Table 4-6: Goldner's Trichrome results from surgical groups .......................................... 64

Table 4-7: Calcein green labeling results from baseline groups ..................................... 65

Table 4-8: Calcein green labeling results from surgical groups ..................................... 66

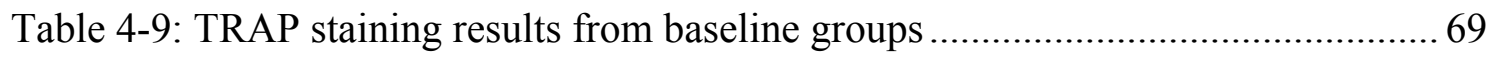

Table 4-10: TRAP staining results from surgical groups ................................................. 69

Table 4-11:ELISA results for soluble pentosidine at time-points 1 and 2 2.................... 72

Table 4-12: ELISA results for soluble pentosidine of surgical groups at all timepoints 72

Table 4-13: ELISA results for soluble CML at time-points 1 and 2........................... 73

Table 4-14: ELISA results for soluble CML of surgical groups at all time-points ........ 73

Table 4-15: ELISA results for sRAGE at time-points 1 and 2 .................................. 75

Table 4-16: ELISA results for sRAGE of surgical groups at all time-points ................. 75

Table 4-17: Carbonylation results for baseline groups .................................................. 77

Table 4-18: Carbonylation results for surgical groups................................................ 78

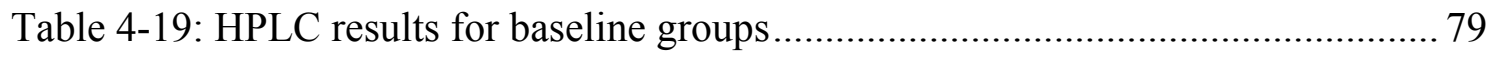

Table 4-20: HPLC results for surgical groups ........................................................... 79

Table 4-21:TRAP/RAGE co-localization results for baseline groups ............................. 81

Table 4-22: TRAP/RAGE co-localization results for surgical groups ........................... 81 


\section{LIST OF ABBREVIATIONS}

aBMD

AGE

ALP

ANOVA

AP-2

$\mathrm{BFR} / \mathrm{BS}$

$\mathrm{BFR} / \mathrm{BV}$

BMD

$\mathrm{BMC}$

BMP

BMU

$\mathrm{BV} / \mathrm{TV}$

CCK

CD

CDB

CD+OVX

CD+SHAM

CML

DCM

DNPH

DXA

dLS

EDTA

ELISA

$\mathrm{H} \& \mathrm{E}$

HFB

HFD

$\mathrm{HF}+\mathrm{OVX}$

$\mathrm{HF}+\mathrm{SHAM}$
Areal bone mineral density

Advanced glycation endproduct

Alkaline phosphatase

Analysis of variance

Activating protein-2

Bone formation rate normalized to bone surface

Bone formation rate normalized to bone volume

Bone mineral density

Bone mineral content

Bone morphogenetic protein

Basic multicellular unit

Percent bone volume

Cholecystokinin

Control diet

Treatment group: baseline rats fed CD

Treatment group: OVX rats fed CD

Treatment group: SHAM rats fed CD

$\mathrm{N}^{\varepsilon}$-carboxymethyllysine

Division of Comparative Medicine

2,4,-dinitrophenylhydrazine

Dual-energy x-ray absorptiometry

Double label surface

Ethylenediaminetetracetic acid

Enzyme-linked immunosorbant assay

Hematoxylin and eosin

Treatment group: baseline rats fed HFD

High fructose diet

Treatment group: OVX rats fed HFD

Treatment group: SHAM rats fed HFD 


\begin{tabular}{|c|c|}
\hline HPLC & High-performance liquid chromatography \\
\hline $\mathrm{I} \kappa \mathrm{B} \alpha$ & $\mathrm{NF}-\kappa \mathrm{B}$ inhibitor alpha \\
\hline $\mathrm{IHC}$ & Immunohistochemistry \\
\hline IL & Interleukin \\
\hline Ir.L.Wi & Interlabel width \\
\hline MAR & Mineral apposition rate \\
\hline M-CSF & Macrophage colony stimulating factor \\
\hline microCT & Micro-computed tomography \\
\hline $\mathrm{MNC}$ & Mononuclear cells \\
\hline MS & Mineralizing surface \\
\hline MS/BS & Percent mineralizing surface \\
\hline MSC & Mesenchymal stem cells \\
\hline MSR & Macrophage scavenger receptor \\
\hline NAFLD & Non-alcoholic fatty liver disease \\
\hline$N F-\kappa B$ & Nuclear factor kappa B \\
\hline NF-IL6 & Nuclear factor-interleukin-6 \\
\hline N.Oc & Osteoclast number \\
\hline NOX4 & NADPH oxidase 4 \\
\hline Oc.RAGE & Osteoclasts expressing RAGE \\
\hline Oc.S & Osteoclast surface \\
\hline OV & Osteoid volume \\
\hline OS & Osteoid surface \\
\hline O.Wi & Osteoid width \\
\hline OVX & Ovariectomy \\
\hline PKC $\beta$ & Protein kinase $\mathrm{c}$ beta \\
\hline RAGE & Receptor for advanced glycation endproduct \\
\hline RAGE $^{-/-}$ & RAGE knockout mice \\
\hline RANKL & Receptor activator of nuclear factor kappa B \\
\hline ROI & Region of interest \\
\hline ROS & Reactive oxygen species \\
\hline SD & Standard deviation \\
\hline
\end{tabular}




$\begin{array}{ll}\text { sLS } & \text { Single label surface } \\ \text { SP-1 } & \text { Specificity factor 1 } \\ \text { sRAGE } & \text { Soluble RAGE } \\ \text { Tb.N } & \text { Trabecular number } \\ \text { Tb.Sp } & \text { Trabecular separation } \\ \text { Tb.Th } & \text { Trabecular thickness } \\ \text { TGF- } \beta & \text { Transforming growth factor beta } \\ \text { TNF- } \alpha & \text { Tumor necrosis factor alpha } \\ \text { TRAP } & \text { Tartrate-resistant acid phosphatase } \\ \text { UV } & \text { Ultraviolet } \\ \text { vBMD } & \text { Volumetric bone mineral density } \\ \text { VOI } & \text { Volume of interest }\end{array}$




\section{INTRODUCTION}

Osteoporosis and the accompanying fractures, is the most prevalent metabolic bone disorder that currently plagues society ${ }^{1}$. According to Osteoporosis Canada, osteoporotic fractures have a greater incidence than heart attack, stroke and breast cancer combined, making it one of the most burdensome diseases*. About 1 in 3 women and 1 in 5 men will suffer from osteoporotic fractures accumulating to a healthcare burden that exceeded $\$ 2.3$ billion in $2010^{*}$. Costs don't stop at the hospital; almost half of the patients who experience fractures do not get released home, and instead are discharged to rehabilitation centers and long-term facilities*. An osteoporotic fracture can also be fatal, with $28 \%$ of women and $37 \%$ of men dying the year after their fracture*. The most common form of osteoporosis is postmenopausal osteoporosis, typically found in women, which is characterized by low bone mass and the deterioration of the microarchitecture of bone due to abnormal bone remodeling occurring after menopause ${ }^{2}$. Although postmenopausal osteoporosis is a prominent disease, the mechanisms behind its development are largely unknown and patients are usually unaware of the weakening of their bones until a fracture has occurred. Confirmation and understanding of the mechanisms behind the disease will lead to new prediction methods and treatments that are important to diagnose the disease and improve the lives of affected populations.

\footnotetext{
*Information obtained from Osteoporosis Canada Website: http://www.osteoporosis.ca/osteoporosis-and-you/osteoporosis-facts-and-statistics/.
} 


\subsection{BIOLOGY OF BONE}

\subsubsection{BONE STRUCTURE}

Bone has many important functions within the body. It has a mechanical role, allowing for the support of body structures; it acts as attachment sites for muscles and as protection for internal organs ${ }^{3}$. Additionally, bone serves a metabolic function, acting like a reservoir for calcium, phosphorus, and growth factors ${ }^{3}$, while also working as the site where hematopoiesis occurs ${ }^{4}$ allowing for the production of blood cells. There are two types of bones found within the adult skeleton: cortical (or compact bone) and cancellous (or trabecular bone) ${ }^{5}$ (Figure 1-1).

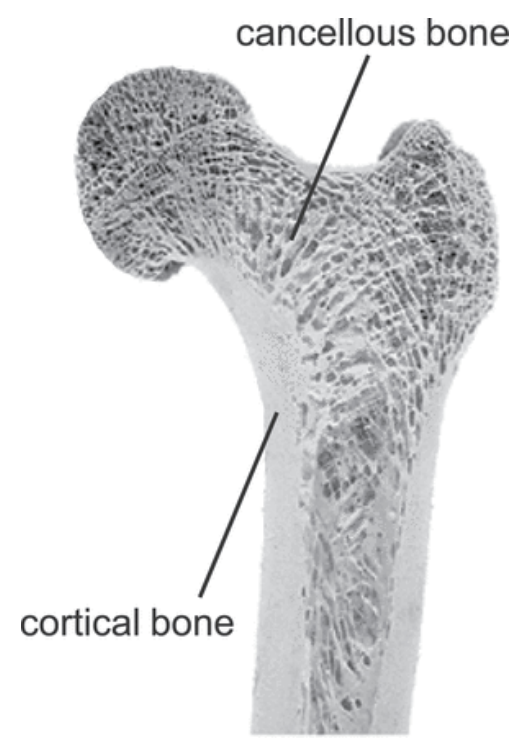

Figure 1-1. Illustration of cortical (compact) bone and cancellous (trabecular) bone. Image obtained from http://ejo.oxfordjournals.org/content/36/4/479.

Cortical bone makes up $80 \%$ of the adult skeleton, and is mainly found in the diaphysis of long bones and surrounding the trabecular bone in the epiphysis ${ }^{5}$. Cortical bone is dense, highly organized, compact bone with low porosity ${ }^{5}$. The remaining $20 \%$ of the adult skeleton is made up of trabecular bone, mainly found within the vertebrae and at the epiphysis and metaphysis of long bones ${ }^{5}$. Trabecular bone is composed of a spongy, 
honeycomb-like structure made of trabecular plates and rods interspersed within the bone marrow ${ }^{6}$. It is highly porous and has a larger bone surface area, causing it to be more metabolically active and thus more vulnerable to skeletal dysfunctions ${ }^{5}$ (Figure 1-2).

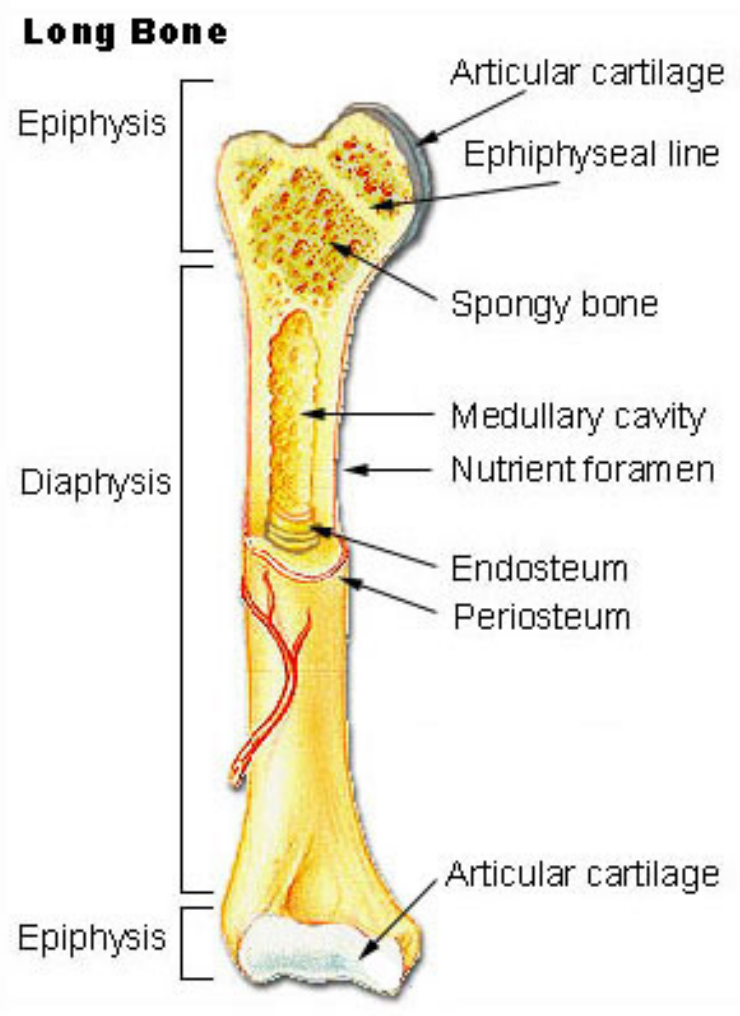

Figure 1-2. Illustration of different regions of long bones. Image obtained from http://www.e-missions.net/cybersurgeons/?/skel_teacher/.

Primary or woven bone is initially laid down during periods of rapid growth and then is remodeled into lamellar bone ${ }^{5}$. Lamellar bone is a hierarchical system made up of concentric layers of lamellae that consist of collagen fibers formed by bundles of collagen fibrils ${ }^{7}$. These collagen fibrils are in turn made up of collagen molecules composed of triple helices of polypeptide chains ${ }^{7}$. In cortical bone these lamellae are formed around Haversian canals and are known as osteons and are the result of remodeling ${ }^{7}$. The canals contain blood, lymphs and nerves, with Volksman's canals running perpendicularly to 
connect them ${ }^{5}$. Trabecular bone also consists of osteons, however they are semilunar in shape and known as packets ${ }^{5}$ (Figure 1-3).

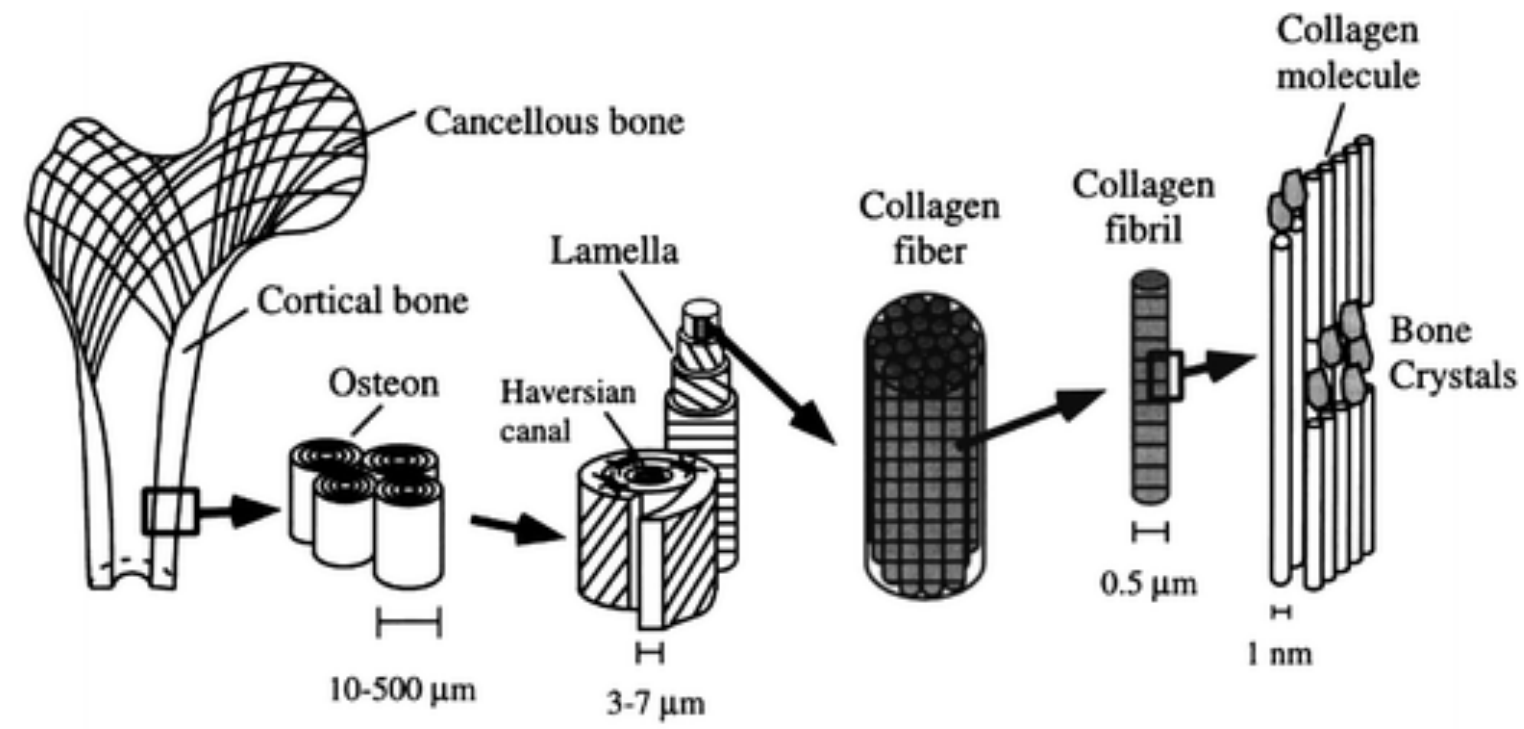

Microstructure

Nanostructure

Macrostructure

Sub-microstructure

Sub-nanostructure

Figure 1-3. Illustration of the hierarchical structure of bone. Image obtained from http://pubs.rsc.org/en/content/articlelanding/2008/jm/b804692a

\subsubsection{BONE COMPOSITION}

Bone is composed of $5-10 \%$ water, $<3 \%$ of lipids, $50-70 \%$ mineral components and $20-40 \%$ organic materials (by weight) ${ }^{8}$. The mineral phase mainly consists of hydroxyapatite $\mathrm{Ca}_{10}\left(\mathrm{PO}_{4}\right)_{6}(\mathrm{OH})$ and small amounts of carbonate, magnesium and acid phosphate; this phase is responsible for mechanical rigidity ${ }^{8}$. The organic phase is mainly comprised of type I collagen and small amounts of other types of collagen, proteins, lipids and cells ${ }^{8}$. It also contains non-collagenous proteins including growth factors and proteoglycans that may regulate matrix deposition and resorption such as osteocalcin and osteopontin $^{8}$. 


\subsubsection{BONE CELLS}

There are three types of bone cells with a role in the maintenance of bone: osteoclasts, osteoblasts and osteocytes. Osteoclasts are bone-resorbing cells; their main function is to break down bone ${ }^{9}$. They are large, multinucleated cells that are derived from hematopoietic stem cells and are made by the fusion of multiple mononuclear precursor cells from the monocyte/macrophage lineage ${ }^{9}$. Osteoclastogenesis is regulated by a multitude of factors including receptor activator of nuclear factor $\kappa \mathrm{B}$ (RANKL) and macrophage colony stimulating factor (M-CSF), and cytokines formed by marrow stromal cells such as interleukin-1 (IL-1) and 6 (IL-6) and parathyroid hormone ${ }^{10}$. Osteoclasts are typically found on the surface of bone, where they bind to osteopontin and bone sialoprotein via $\alpha{ }_{v} \beta_{3}$ integrins ${ }^{11}$. Osteoclasts become polarized upon binding to the bone matrix and form an actin ring around the perimeter of the attachment site ${ }^{12}$. This isolates the resorption compartment from the surrounding bone surface, allowing this area to acidify to a pH of approximately 4.5 through the release of hydrogen and chloride ions ${ }^{13}$. The surface attached to the bone becomes a ruffled border as vesicles containing matrix metalloproteinases and cathepsin $\mathrm{K}$ are transported to the membrane ${ }^{13}$. The acidic environment dissolves the bone mineral and exposes the organic matrix for cleavage by enzymes released from the osteoclasts ${ }^{13}$.

Osteoblasts are bone-forming cells; involved in the synthesis of new bone matrix on bone forming surfaces ${ }^{8}$. They are derived from mesenchymal stem cells found in the bone marrow and their differentiation is regulated by bone morphogenetic protein (BMP), transforming growth factor (TGF- $\beta$ ), interlukein-3 (IL-3) and IL-6 ${ }^{8,14-16}$. They are known to express alkaline phosphatase (ALP) during early stages of differentiation and 
osteocalcin in later stages of differentiation ${ }^{14}$. Active, mature osteoblasts are mononuclear cuboidal cells that are often found tightly packed along the surface of the bone and secrete type I collagen and other matrix proteins to synthesize new organic matrix ${ }^{14}$.

Osteocytes are terminally differentiated osteoblasts that are trapped within lacunae in mineralized bone ${ }^{17}$. Their function is to act as mechanosensors, in which they transduce mechanical stress placed upon the bone into biological activity ${ }^{17}$. Osteocytes have multiple cellular processes that connect to other osteocytes in the bone through canaliculi channels that allow them to communicate with each other and the bone surface in response to mechanical loading ${ }^{18}$. They are thought to express matrix proteins that support intracellular binding, as well as play a role in cell signaling to initiate boneremodeling ${ }^{17}$.

\subsubsection{BONE MINERALIZATION}

Bone mineralization is essential for the hardness and strength of bone and occurs in two steps. The first is the deposition of the organic matrix by osteoblasts. This matrix is known as osteoid as consists of mainly type I collagen and smaller amounts of noncollagenous proteins such as proteoglycans and osteopontin ${ }^{3}$. Next, mineralization occurs in two phases; a vesicular and a fibrillar phase ${ }^{3,8}$. The vesicular phase consists of the release of matrix vesicles from osteoblasts that allow for accumulation and nucleation of calcium and phosphate to allow hydroxyapatite formation ${ }^{3,8}$. The fibrillar stage includes mineral deposition between the ends of collagen fibres. As bone matures, the hydroxyapatite crystals enlargen, aggregate and purify; processes that may be facilitated 
by bone matrix macromolecules and lead to formation of mineralized bone ${ }^{8}$.

Bone is constantly being remodeled at different times and locations within the skeleton. This leads to variation and heterogeneity of the material properties of bone ${ }^{19}$. Mineralization changes with age and disease, and the biological determinant of mineralization is bone turnover ${ }^{20}$. Hypomineralization is defined as having lower mineral densities and is often seen in periods of rapid growth and high turnover, such as during osteoporosis and calcium deficiency. Hyperminerilzation is defined as having higher mineral densities and is often seen during aging, low bone remodeling, and when taking drugs such as bisphonantes ${ }^{19,20}$.

\subsubsection{BONE REMODELING}

Bone is a dynamic tissue constantly undergoing an important process known as bone remodeling or bone turnover. This process is essential for the healthy maintenance of bone and allows for adaptation to mechanical loads ${ }^{21}$. Older bone is replaced, microcracks are repaired and a mineral homeostasis is maintained through this important process ${ }^{21}$. Osteoclasts and osteoblasts form Basic Remodeling Units (BMU) and are responsible for the breakdown of packets of bone, followed by the formation of bone matrix, and subsequent mineralization of new bone ${ }^{22}$. It is important during this time that the activity of the osteoclasts and osteoblasts are coupled, so the same amount of bone resorbed by osteoclasts is formed by osteoblasts ${ }^{23}$.

Bone remodeling consists of four sequential phases: activation, resorption, reversal and formation (Figure 1-4). Activation involves the recruitment and activation of monocyte-macrophage osteoclast precursors from the circulation to the targeted area of 
bone $^{24}$. These cells merge together to form a mature, multinucleated osteoclast and bind to the target bone to create a sealing zone as mentioned previously ${ }^{24}$. During the resorption phase osteoclasts secrete several factors including tartrate-resistant acid phosphatase (TRAP), cathepsin $\mathrm{K}$, and matrix metalloproteinases that digest the organic matrix as the acidic environment breaks down the mineral phase ${ }^{25}$. The resorptive phase typically takes 2 to 4 weeks and results in resorption pits or Howship's lacunae on the surface of trabecular bone and longitudinal tunnels in cortical bone ${ }^{8}$. Osteoclasts then undergo apoptosis and the reversal phase commences ${ }^{26}$. During this phase multiple cells are present in the resorption pit including monocytes and preosteoblasts, which are recruited to begin bone formation ${ }^{8}$. The coupling signals that link the end of the resorption phase to the beginning of the formation phase are largely unknown, although several theories are being investigated. Coupling signals could be from growth factors or cytokines that may be released from resorbed bone such as TGF- $\beta$ and BMPs ${ }^{27}$, or from factors released from osteoclasts, including cardiotrophin- ${ }^{28}$, semaphoring $4 \mathrm{D}{ }^{29}$ or EphrinB2 ${ }^{30}$.

The final phase is formation in which mature osteoblasts synthesize new collagenous organic matrix that will eventually mineralize into bone ${ }^{8}$. They have also been found to release small, membrane-bound matrix vesicles to assist with concentration of calcium and phosphate, while impeding mineralization inhibitors ${ }^{31}$. This phase is the longest, and takes approximately 3 months to complete ${ }^{8}$. After this time osteoblasts undergo one of three fates: apoptosis, become embedded in the bone and develop into osteocytes or become bone lining cells ${ }^{8}$. 


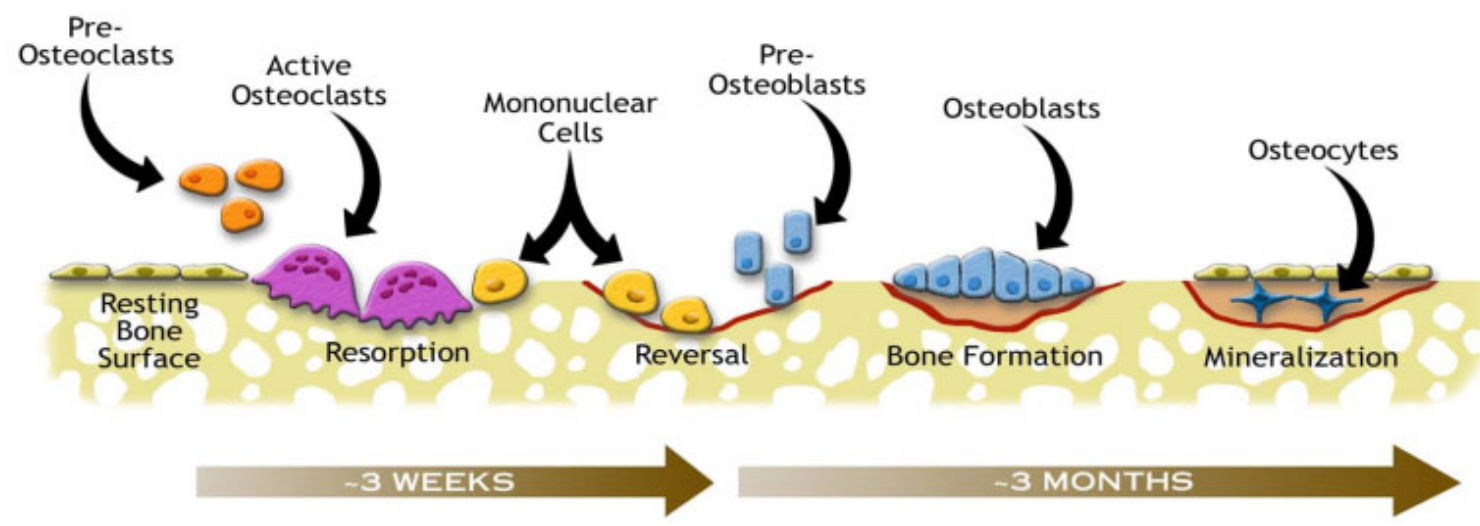

Figure 1-4. Illustration of the bone remodeling cycle. Image obtained from http://www.ns.umich.edu/Releases/2005/Feb05/bone.html.

In an ideal situation, the amount of bone resorbed is equal to the amount formed and there is no net bone loss or gain ${ }^{23}$. This is achieved through the work of many cytokines, mechanical stresses and a variety of hormones ${ }^{32}$. During development of bone in childhood, the formation outweighs the resorption to allow for the lengthening and strengthening of bone ${ }^{33}$. During adulthood, we experience a healthy balance of resorption and formation until we reach peak bone mass at around 30 years of age and the balance is offset with resorption can outweigh formation ${ }^{33}$. This is seen through a decreasing amount of osteoid deposited as we age. This is possibly due to a decrease in osteoblast precursors, a decrease in stem cells that the precursors are derived from, a decrease in differentiation signals or a shortening of the lifespan of osteoblasts ${ }^{33}$. Due to the decline in bone formation, the imbalance gradually leads to a net bone loss, which can play a role in the development of osteoporosis ${ }^{34}$.

\subsection{OSTEOPOROSIS}

Osteoporosis is a metabolic bone disease characterized by low bone mass and the 
deterioration of microarchitecture ${ }^{35}$. This causes the skeleton to be very fragile and highly susceptible to fracture ${ }^{35}$. There are two types of osteoporosis: primary and secondary osteoporosis. Secondary osteoporosis is caused by chronic conditions that lead to acceleration of bone loss such as hyperthyroidism or medication-related inducement caused by long-term use of drugs such as glucocorticoids ${ }^{1}$. Secondary osteoporosis accounts for about $10 \%$ of cases, while the majority of cases are classified as primary osteoporosis ${ }^{1}$. Primary osteoporosis is further characterized into two types; Type I or postmenopausal osteoporosis and Type II or age-related osteoporosis ${ }^{1}$. Postmenopausal osteoporosis is the most common and affects mostly women after the reduction of estrogen production experienced during menopause ${ }^{2}$. Age-related osteoporosis affects both men and women equally and is caused by normal loss of bone over time; symptoms usually manifest around 75 years of age or older ${ }^{1,34}$.

A commonly used and widely applied clinical measurement of bone quality and risk of fracture is dual-energy X-ray absorptiometry (DXA) ${ }^{36}$. DXA is a non-invasive measurement utilizing X-ray beams to measure bone mineral density ${ }^{36}$. DXA results are used by the World Health Organization's definition of osteoporosis and osteopenia, with a T-score of below -2.5 signifying the presence of osteoporosis ${ }^{36}$. 


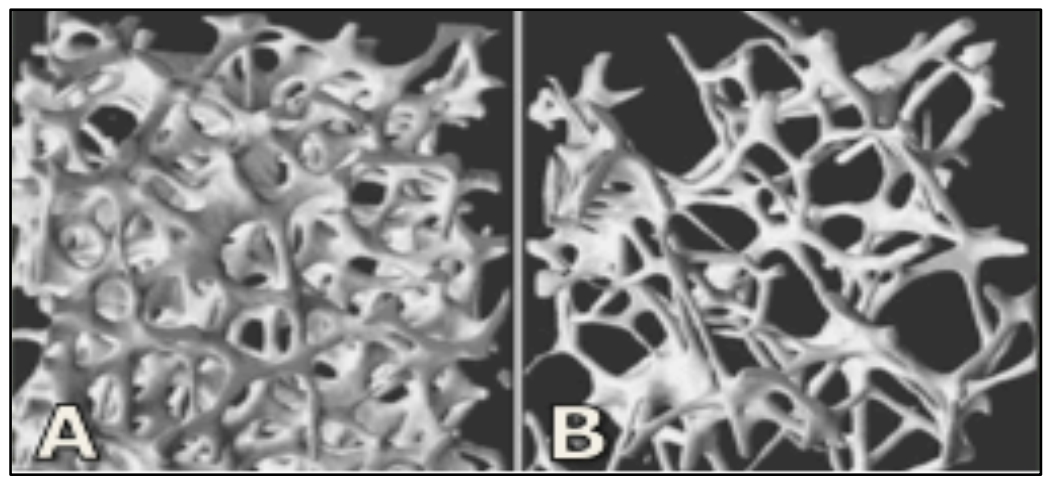

Figure 1-5. Microcomputed tomography images of trabecular bone from the vertebrae of a normal woman (A) and an age-matched osteporotic woman (B). Image obtained from http://phar.uokufa.edu.iq/staff/Mohammad/lectures.html

\subsubsection{POSTMENOPAUSAL OSTEOPOROSIS}

Postmenopausal osteoporosis is the most common form of osteoporosis, and mainly affects women ${ }^{2}$. Following menopause, women experience a steep increase in bone loss, occurring at approximately $5 \%$ per year for about $5-10$ years ${ }^{23}$. During this time, more BMUs are created, and both bone resorption and bone formation are enhanced due to coupling ${ }^{23,26,37}$. However, the enhanced number and activity of osteoblasts is insufficient in combating the heightened amount of bone resorption, therefore a net bone loss is experienced ${ }^{23,38,39}$.

The classical explanation of this accelerated bone loss is the depletion of estrogen levels following the arrest of ovarian function causing an imbalance in bone remodeling 40. This is based on many years of research that have shown estrogen to have an essential role in the activation and regulation of bone remodeling; made evident through estrogen hormone replacement, which is able to combat bone loss experienced after ovariectomy 41. There are many hypotheses as to how estrogen depletion leads to this increased remodeling rate including increased release of cytokines associated with heightened 
osteoclastogenesis and osteoclast activity such as tumor necrosis factor alpha (TNF- $\alpha$ ), IL-1 and IL- $6{ }^{42-44}$. Estrogen has also been found to have a pro-apoptotic effect on osteoclasts while having an anti-apoptotic effect on osteoblasts ${ }^{44,45}$. Therefore in theory, when estrogen is depleted osteoclasts live longer allowing them to resorb more bone, while osteoblasts have a shorter lifespan, leading to insufficient bone formation ${ }^{41,43}$.

Estrogen depletion also varies between women; some will decrease production earlier, while others may experience a more dramatic decrease at one period. The variance with estrogen depletion can have an effect of the fracture risk and bone density ${ }^{46}$. In a study by Greendale et al, they measured estradiol levels from postmenopausal women and found that total estrogen levels were related to bone density at the spine, hip and forearm 46. The lower the levels of estrogen, the lower the bone density, and the greater risk of fracture. Therefore, the level of circulating estrogen is an important factor to be considered in the development of postmenopausal osteoporosis ${ }^{46,47}$.

\subsection{NEW THEORY OF POSTMENOPAUSAL OSTEOPOROSIS}

There is very convincing evidence to the critical role estrogen plays in bone remodeling and the development of postmenopausal osteoporosis. However, all women who go through menopause experience the depletion of estrogen and the subsequent bone loss that accompanies it, but only $25-30 \%$ of women will actually develop postmenopausal osteoporosis and its accompanying fractures**. Therefore there must be

\footnotetext{
**Information obtained from The Bone and Joint Decade. The Burden of Musculoskeletal Diseases in the United States.www.boneandjointburden.org/. 2008.
} 
some other mechanism in place that is working in tandem with estrogen deficiency to cause the development of this disease in certain women. A theory that is under investigation suggests sex steroid depletion is not the direct cause of the increase in bone remodeling, but rather works indirectly by increasing reactive oxygen species (ROS) thereby causing oxidative stress, which can subsequently alter bone remodeling ${ }^{40}$.

There is growing evidence of increased levels of pro-inflammatory cytokines, prooxidant markers and oxidative stress markers in postmenopausal women compared to premenopausal women that show this hormone also works as an antioxidant ${ }^{48}$. Estrogen levels positively correlate with plasma antioxidant capacity and expression of antioxidant enzymes such as catalase and glutathione peroxide ${ }^{49}$, while negatively correlating with lipid peroxidase levels ${ }^{50}$. The proposed role of estrogen as an antioxidant may be related to free radical scavenging activity, inhibiting oxidative modifications of lipoproteins and phospholipids and the ability to influence antioxidant enzyme activity ${ }^{50}$.

The possibility of an increase in oxidative stress due to a decline in estrogen production on bone loss experienced during postmenopausal osteoporosis is an active area of research. Goettsch et al. investigated NADPH oxidase 4 (NOX4), an enzymatic source of ROS within the body ${ }^{51}$. A mutation of the NOX4 gene was associated with altered parameters of bone metabolism in middle-aged women, and patients with untreated osteoporosis experienced an increased expression of NOX4 within their bones ${ }^{51}$. When ovariectomies were performed on control mice, an increased expression of NOX4 was observed, while in NOX4 knock-out mice there was inhibited bone loss as compared to the control groups ${ }^{51}$. This data suggests that the loss of estrogen production may have lead to an increase in NOX4, subsequently increasing the production of ROS, which 
could potentially have a role in increased bone loss ${ }^{51}$. A study by Almeida et al., presented the effects of estrogen on $\mathrm{p} 66^{\mathrm{shc}}$, an adaptor protein that amplifies mitochondrial ROS generation and leads to hydrogen peroxide (a common ROS)-induced apoptosis of osteoblasts ${ }^{52}$. When protein kinase $\mathrm{c}$ beta $(\mathrm{PKC} \beta)$ is activated by ROS it works to phosphorylate and subsequently activate P66 ${ }^{\text {shc }}$. However, when cells were exposed to estrogen, PKC $\beta$ phosphorylation was blocked and an increase in ROS levels and osteoblast apoptosis were not experienced ${ }^{52}$. Another study by Almeida et al. found that estrogen and NAC, an antioxidant, treatment of male and female rats that had undergone gonadectomies prevented the decrease in bone mineral density and the increase in ROS that was seen in the control groups. This suggests that estrogen may act similarly to an antioxidant, and help to reduce the production of $\operatorname{ROS}^{41}$.

While the exact mechanism behind oxidative stress affecting bone remodelling is relatively unknown, recent evidence points to one particular compound that is increased in aged bone; advanced glycation endproducts (AGEs). AGEs are compounds formed in long-lived proteins as the result of non-enzymatic glycation reactions by reducing sugars leading to formation of cross-links and protein adducts ${ }^{53}$. Their formation is driven forward by oxidation through ROS, and osteoporotic patients have been found to have a greater amount of AGEs ${ }^{54,55}$. AGEs have also been found to have a negative correlation with bone density, a positive correlation with the Singh index (index of osteoporosis severity) ${ }^{55}$. The presence of AGEs has been demonstrated to enhance osteoclast-mediated resorption in vitro ${ }^{56}$ as well inhibiting bone formation by osteoblasts in vitro ${ }^{57}$. The effect of AGEs on the processes of these bone cells is likely due to binding to the Receptor for Advanced Glycation Endproducts (RAGE) a multiligand member of the immunoglobulin 
superfamily of cell surface receptors ${ }^{58}$. When AGEs bind to RAGE intracellular signalling pathways are activated, which trigger the creation and release of several proinflammatory factors ${ }^{58,59}$. A positive feedback loop has been proposed by which RAGE ligation by AGEs leads to inflammation, increased oxidative stress, and subsequent AGE formation ${ }^{54,58}$. Therefore, estrogen may play a role as an antioxidant, and, without it, there is increased ROS expression driving the production of AGEs and ligation of RAGE. This interaction could cause changes in the function and regulation of the bone cells, which could potentially drive the formation of postmenopausal osteoporosis.

\subsubsection{ADVANCED GLYCATION ENDPRODUCTS}

AGEs form as the result of non-enzymatic glycation reactions in which reducing sugars (e.g. glucose and fructose) and carbohydrate metabolites (e.g. dicarbonyls) undergo direct chemical reactions with proteins leading to the adduction of a carbohydrate to other biomolecules ${ }^{60}$. AGEs are naturally occurring and accumulate with age in long-lived extracellular proteins such as the aorta, skin and bone collagen ${ }^{54,60-62}$. AGEs can be categorized as cross-linking agents between two adjacent molecules and side-chain modifications that can alter the profile of the molecule ${ }^{53,60}$. When disadvantageous crosslinking occurs in bone collagen molecules a large effect is observed on the optimal function and mechanics of the collagen protein ${ }^{53,54}$. AGE accumulation can also cause deterioration of bone tissue quality including increased stiffness of collagen and decreased solubility, elasticity, and proteolytic digestion; which can affect tissue remodelling ${ }^{54}$. Over twenty different kinds of AGEs have been discovered, but the most characterized and studied are pentosidine, a cross-linking AGE, and $\mathrm{N}^{\varepsilon}$ - 
carboxymethyllysine (CML) a protein adduct (Figure 1-6). Both serve as well-accepted biomarkers for AGEs ${ }^{60,63}$.

The formation of AGEs is a multistep process known as the Maillard reaction and can lead to the development of glycation and glycoxidation products. Both products are produced from identical early stages ${ }^{53}$. Reducing sugars and/or carbohydrate metabolites react with N-terminal amino groups (usually a lysine) of proteins to form reversible Schiff bases (aldimines). The Schiff bases reversibly rearranges to form Amadori compounds such as fructose-lysine. At this point the compounds can undergo further chemical modifications including dehydration, $\beta$-eliminations and condensation reactions to create AGEs such as pyrraline ${ }^{60}$. Alternatively, the Amadori products can undergo oxidation by ROS leading to the formation of glycoxidation AGEs such as CML and pentosidine (Figure 1-7) ${ }^{53,60}$.
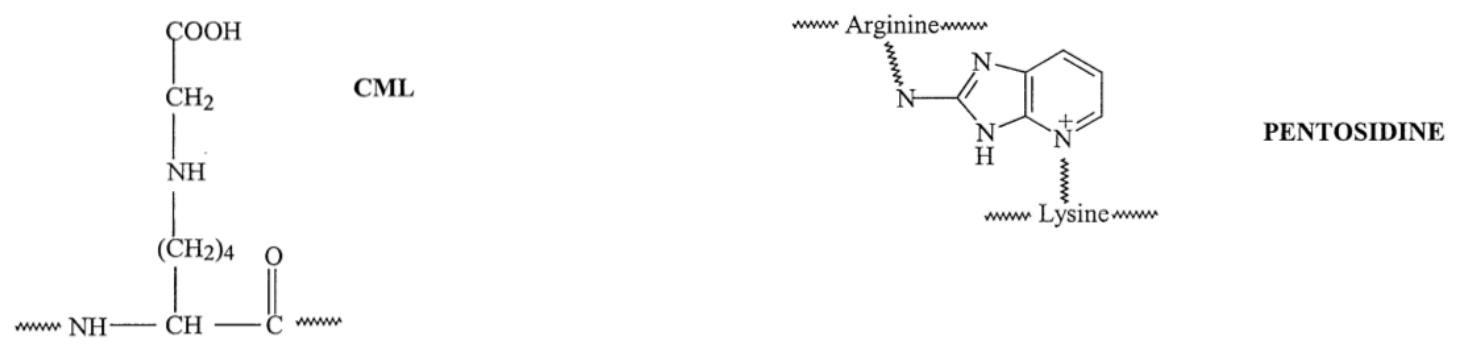

Figure 1-6. Structures of $\mathrm{N}^{\varepsilon}$-carboxymethyllysine (CML) and pentosidine. Images obtained from reference ${ }^{60}$. 


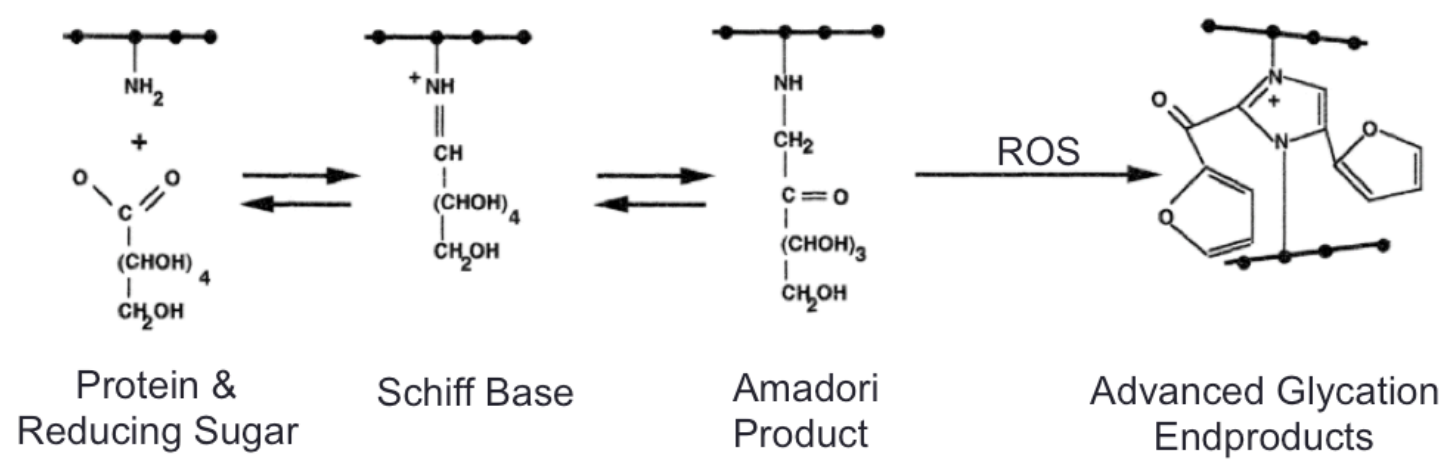

Figure 1-7. Schematic of the Maillard reaction showing the formation of an advanced glycation endproduct crosslink. Image modified from reference ${ }^{64}$.

Several studies have indicated that AGE production stems from three different factors. Firstly, since AGEs take a period of weeks to form, proteins that are long-lived are targeted as they have a longer exposure time for chemical reactions ${ }^{60}$. AGE creation is also dependent on the relative sugar concentrations in the early stages of the Maillard reaction ${ }^{60}$. Finally, the development is dependent on the local oxidative environment, with more oxidative stress accelerating the generation of AGEs ${ }^{54}$. Therefore, in incidences where more ROS are available, such as after menopause ${ }^{49,64}$ there is a greater driving force for the production of AGEs ${ }^{60}$. This is similarly seen in other diseases, such as diabetes, where oxidative stress is high and an increase in AGEs is also experienced ${ }^{65}$. It has also been observed that the production of AGEs leads to the formation of more ROS, which can contribute to the production of more AGEs and oxidative stress ${ }^{60}$. Therefore, there is increasing evidence that AGEs may participate in or activate physiological responses to oxidative stress, including inflammation and hyperglycaemia $54,60,64$. 


\subsubsection{RECEPTOR FOR ADVANCED GLYCATION ENDPRODUCTS}

AGEs have several different receptors including macrophage scavenger receptors (MSR) and AGE-1, -2 , and $-3{ }^{66}$ but the most characterized is RAGE ${ }^{58}$. RAGE is a multiligand member of the immunoglobulin superfamily of cell surface receptors composed of three immunoglobulin-like regions: one "V" type domain, and two "C" type domains, as well as a short transmembrane domain and a 43 -amino acid cytoplasmic tail ${ }^{67}$. The "V" type domain is responsible for ligand binding; while the cytoplasmic tail is used for intracellular signalling ${ }^{67}$. The transcription of RAGE is mediated by specificity protein-1 (SP-1), activating protein-2 (AP-2), nuclear factor kappa B (NF- $\mathrm{KB})$ and nuclear factor IL6 (NF-IL6), and it can be both constitutively expressed and induced depending on the cell-type and developmental stage ${ }^{67,68}$. RAGE is constitutively expressed in skin and lung tissues as well as during embryonic development ${ }^{66,67}$. Under physiological conditions in most adult tissues, its expression is down regulated; however it can be upregulated during times of inflammation ${ }^{66,67}$. RAGE binds to many ligands including but not limited to: AGEs, amyloid-beta peptide, S100/calgranulins, and HMGB1 ${ }^{67,69,70}$. Interestingly, most of its ligands accumulate in tissues during aging, diseases, inflammation and host responses ${ }^{67}$. RAGE has been found to expressed on a variety of cells including but limited to; monocytes/macrophages, endothelial cells, neuronal cells ${ }^{68}$, osteoclasts $^{71}$, osteoblasts ${ }^{72}$ and mesenchyal stromal cells ${ }^{73}$.

RAGE can also be found in truncated, secreted forms missing the trans membrane domains and cytosolic tail and is known as soluble RAGE (sRAGE). sRAGE can be produced through alternative splicing of the RAGE pre-mRNA, or through proteolytic cleavage ${ }^{67,68,70}$. The functional significance of these secreted forms is unknown, however 
one proposed mechanism is the blockade of RAGE by acting as a decoy receptor, competing with the full-length RAGE for ligand binding and subsequently blunting

RAGE signalling ${ }^{74-76}$. Synthetically produced versions of sRAGE are commonly used in experiments, and successfully act as a blockade of RAGE signalling ${ }^{67,69}$.

\subsection{AGE-RAGE INTERACTION}

When AGEs bind to RAGE, intracellular signalling pathways are activated which trigger the creation and release of several pro-inflammatory factors, most notably NF- $\mathrm{NB}$. $\mathrm{NF}-\mathrm{kB}$ is a key transcription controller, linking immune responses to inflammation and infection ${ }^{68}$. NF- $\mathrm{kB}$ exists in the cytosol in its inactive form attached to the inhibitor molecule I $\mathrm{B} \mathrm{B} \alpha$. When RAGE is engaged it activates phosphatidylinositol-3-kinase, KiRas and mitogen-activated protein kinases ERK1 and ERK2, which phosphorylate and degrade I $\mathrm{B} \alpha \alpha$ resulting in the release and translocation of $\mathrm{NF}-\kappa \mathrm{B}^{58,67,68}$. Upon activation, NF- $\kappa \mathrm{B}$ binds to decameric DNA sequences to activate the transcription of genes it regulates which include RAGE itself and $\mathrm{I} \kappa \mathrm{B} \alpha^{58}$. In normal NF- $\kappa \mathrm{B}$ expression, the creation of I $\mathrm{B} \alpha$ leads to self-termination within minutes to hours ${ }^{58}$. However, when NF$\kappa \mathrm{B}$ is activated through RAGE ligation, NF- $\kappa \mathrm{B}$ p65 mRNA levels are also elevated. This overwhelms the newly synthesized inhibitor and leads to maintained NF- $\kappa B$ activation ${ }^{64}$. Since RAGE itself is also regulated by NF- $\kappa$ B this prolonged release leads to upregulation of the receptor and further amplification of the signals produced by NF- $\kappa \mathrm{B}$, including inflammatory cytokines like IL-6, IL-1 and TNF $\alpha^{56,58,69,77}$. These factors play a significant role in regulation and formation of osteoclasts and osteoblasts ${ }^{8,10,14}$. In 
addition, AGE-RAGE interaction causes the release of ROS through the activation of NADPH oxidases, further lending to the oxidative stress of the local environment ${ }^{78,79}$.

\subsubsection{AGE-RAGE AND OSTEOCLASTS}

Since RAGE is expressed in both osteoblasts and osteoclasts it can potentially have direct effects on both of these cell types. The effects that AGEs and /or RAGE have on osteoclasts has been documented by several groups. Miyata et al., looked at the effect of AGEs on osteoclast-induced bone resorption in vitro using a pit formation assay. They did this by exposing unfractionated bone cells containing TRAP-positive mononuclear cells (MNC) in the presence of AGEs, and found an increased number of resorption pits on the dentin slices they were plated on. However, the number of TRAP-positive MNC was not increased, suggesting that the AGEs caused a greater activation of pre-existing cells ${ }^{80}$. Dong et al. looked at the amount of bone resorption due to AGEs by plating osteoclasts directly onto human bone slices from donors of a variety of ages. They found that the higher the matrix AGE concentration, the more resorption pits and resorptive areas ${ }^{81}$. Ding et al, looked at the in vivo role of RAGE in bone remodelling and bone loss using RAGE knockout mice $\left(\mathrm{RAGE}^{-/}\right)$. They found $\mathrm{RAGE}^{-/-}$had significantly increased bone mineral density, biomechanical strength as well as decreased osteoclast formation and serum levels of IL-6. They also looked at the effects of $\mathrm{RAGE}^{-/-}$on bone loss seen after ovariectomy and they found that $\mathrm{RAGE}^{-/}$mice were resistant to bone loss usually induced by ovariectomy ${ }^{82}$. A study by Lalla et al., confirms these results as they found that blockade of RAGE using sRAGE led to diminished levels of alveolar bone loss, as well as lowered levels of TNF- $\alpha$ and IL- $6^{77}$. Zhou et al. also found increased bone mass 
and bone mineral density as well as decreased bone resorption in $\mathrm{RAGE}^{-/-}$mice. They also looked at the role of RAGE in osteoclast maturation and function using osteoclast cells derived from $\mathrm{RAGE}^{-/-}$mice and found these osteoclasts were morphologically and functionally impaired, possibly due to disrupted actin cytoskeletal organization ${ }^{71}$. These results taken together implicate an important role for AGEs and RAGE in osteoclast formation and activity in physiological processes as well as in times of inflammatory responses. Therefore the receptor and it ligand can be essential players in the bone loss seen in postmenopausal osteoporosis, especially if ligation causes the upregulation of osteoclast activity.

\subsubsection{AGE-RAGE AND OSTEOBLASTS}

Several groups have also investigated the roles of AGEs and/or RAGE on several processes concerning osteoblast cells. Stolzing et al. investigated the differentiation of mesenchymal stem cells (MSC) into osteoblasts. They found that MSCs retrieved from diabetic rats exhibited elevated levels of AGEs and RAGE expression, and as a result had a reduced osteogenic differentiation potential in vitro ${ }^{83}$. McCarthy et al. investigated the proliferation, differentiation and mineralization of osteoblast-like cells. When these cells were plated on an AGE-matrix, there was decreased expression of ALP in maturing osteoblasts as well as diminished nodule formation in the mineralizing stage of development. This suggests that that accumulation of AGEs on bone extracellular matrix may regulate osteoblastic cells ${ }^{78}$. In another study by McCarthy et al., osteoblastic attachment to an AGE modified type-I collagen matrix was investigated. They found that osteoblast-like cells were inhibited from binding to this matrix most likely due to altered 
$\alpha$ and $\beta$ subunits of integrin receptors ${ }^{57}$. Sanguineti et al., also show this by presenting data confirming the inhibition of ALP and col-I $\alpha 1$ expression, as well as flawed nodules and osteoblast function when human osteoblast cells were incubated with pentosidine ${ }^{84}$. Franke et al. investigated the effects of glycated bovine serum albumin on the mRNA expressions of various genes in human osteoblast cultures. They found that the AGERAGE pathway was activated, as they saw RAGE mRNA upregulation, and enhanced RANKL production with impaired matrix mineralization and down regulation of ALP and osteocalcin ${ }^{85}$. Finally, a study by Alikhani et al. investigated the effects of CML-rich collagen on osteoblast cell cultures. They found that CML-collagen stimulated the apoptosis of bone-lining cells in vivo and of osteoblast cells in vitro. They plated osteoblasts in the presence of a RAGE inhibitor and found a decrease in cell apoptosis and therefore concluded that AGE/RAGE binding could have an apoptotic effect in osteoblast cells ${ }^{86}$. These results show that AGEs and/or RAGE have an effect on osteoblasts, potentially hindering their ability to form and produce a collagen matrix, thereby possibly contributing to the bone loss seen in postmenopausal osteoporosis 


\subsection{PROPOSED MECHANISM}

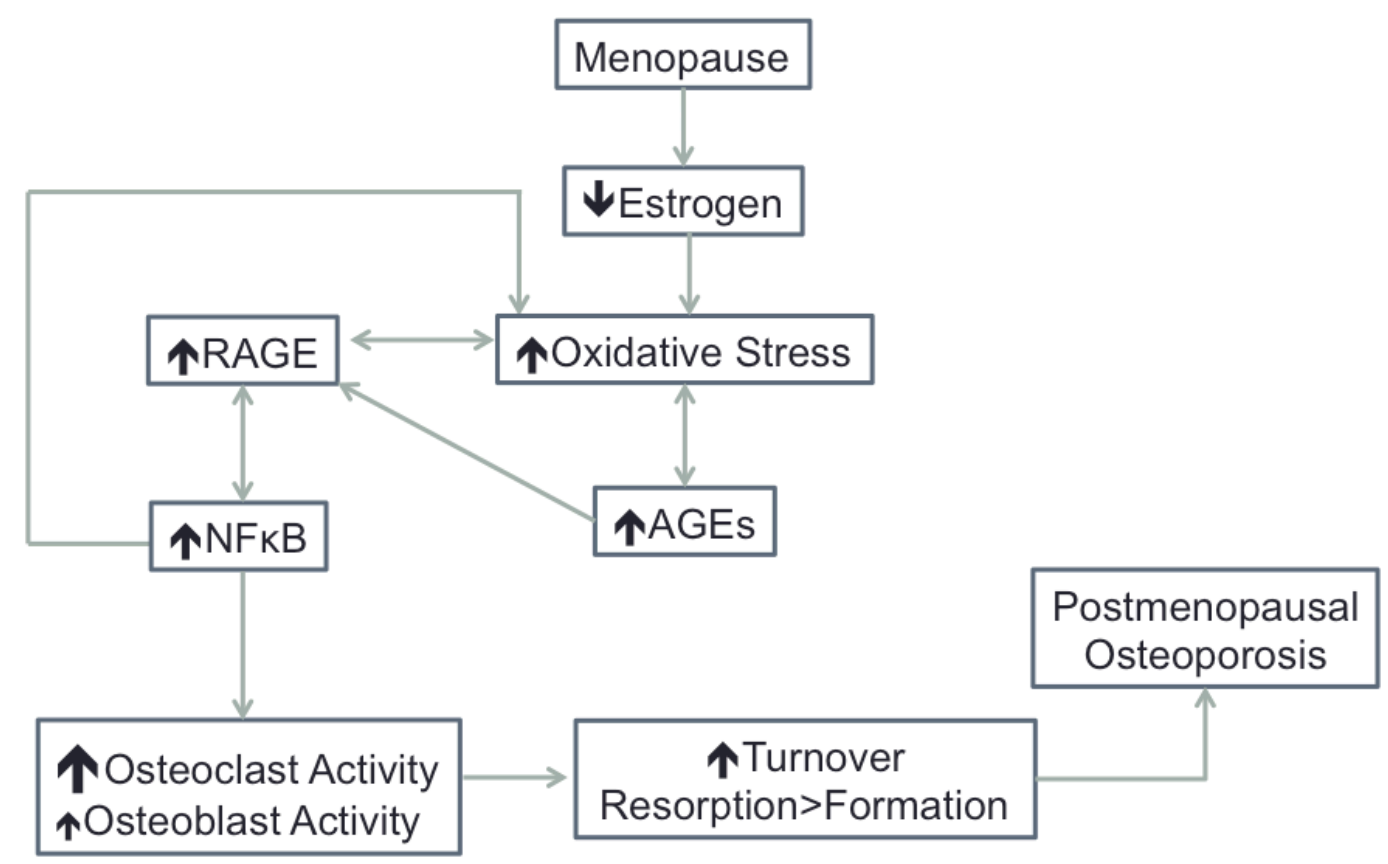

Figure 1-8. Proposed mechanism.

Based on recent literature we have proposed a mechanism in which the decrease in estrogen experienced in postmenopausal women can lead to postmenopausal osteoporosis via AGEs and their interaction with RAGE. Firstly, a woman will undergo menopause leading to the deficiency of production of ovarian hormones such as estrogen. The decrease in estrogen levels will lead to an increase in oxidative stress in the body. This increase in oxidative stress will lead to an accelerated production of AGEs, which will in turn lead to the production of more oxidative stress. AGEs will then exert their effect through binding to RAGE, a process which again produces more oxidative stress. The ligation of RAGE will cause the activation of $\mathrm{NF}-\kappa \mathrm{B}$, which in turn upregulates RAGE, as well as increasing oxidative stress. AGE-RAGE ligation will then affect osteoclast and osteoblast activity, possibly because of the release of several pro-inflammatory cytokines 
released due to NF- $\kappa \mathrm{B}$ activation or through the presence of AGEs in the type I collagen matrix. This will lead to an increase in bone turnover, in which resorption exceeds formation, leading to bone loss and potentially postmenopausal osteoporosis and fractures.

\subsection{ANIMAL MODELS}

\subsubsection{RAT OVARIECTOMY MODEL}

The rat ovariectomy model (OVX) is a well-established and commonly used model for the study of pathogenesis and treatment of osteoporosis ${ }^{87,88}$. The rat is often used because they are low-cost to maintain, easy to handle, and exhibit similar skeletal biology and osteopenia to humans ${ }^{89}$. A limitation to this model is lack of Haversian canals in rat bone, instead of seeing remodelling of cortical bone in the canals, they experience cortical bone loss at the endosteum, and cortical bone gain in the periosteum

89. Another limitation is the model does not experience the fragility fractures that are the hallmark of postmenopausal osteoporosis ${ }^{89}$. Therefore, the bone loss seen in this model is better described as osteopenia rather then osteoporosis ${ }^{87,90}$.

The OVX rat model consists of the bilateral removal of the ovaries leading to ovarian hormone deficiency ${ }^{90}$. Similar to humans, this results in the rapid loss of cancellous bone mass and strength through an increase in bone turnover, and is followed by a steady state of bone loss ${ }^{88}$. In the rat model, not all cancellous bone sites are affected in the same fashion, for example rapid bone loss occurs in the proximal tibial metaphysis between days 14 and 90 post-OVX, while lumbar vertebral bodies experience rapid bone loss between days $60-270^{89,90}$. As in humans, there is a greater impact on cancellous bone then cortical bone and changes in cortical bone occur very slowly ${ }^{90}$. 
There are two different age groups that the OVX surgery can be performed on. The first is the aged rat model, in which the surgery is performed on rats that have reached skeletal maturity ${ }^{87}$. The age range for this model is $6-12$ months of age, because by 6 months changes in femur density were minimal in the rats, while by 12 months all bone parameters measured had reached peak levels ${ }^{87}$. Using this model ensures that any skeletal changes post-OVX are due to ovarian hormone deficiency. The second model is the mature rat model, in which the surgery is performed on rats that have reached sexual maturity so they can adequately respond to sex hormone deficiency ${ }^{87}$. While these rats may not be skeletally mature, the effects of OVX manifest quicker then the aged model and the characteristics of bone loss are similar to the aged model. Both models are actively used in research ${ }^{87}$.

\subsubsection{HIGH FRUCTOSE DIET MODEL}

AGEs are often formed in hyperglycaemic conditions because their creation is dependent on reducing sugar concentrations ${ }^{60}$. When more reducing sugars are present, more spontaneous non-enzymatic reactions occur. Among the reducing sugars that can elicit the Maillard reaction are glucose, fructose and sucrose, all of which are naturally found within the body ${ }^{62}$. While glucose is often present in the largest amounts in vivo, fructose is a much more potent initiator of the Maillard reaction ${ }^{62}$. The high fructose diet has been used in many studies, and has been shown to elicit increased levels of AGEs in tissues of the rat including bone, skin, tendon, aorta and other vasculature ${ }^{61,62,91-93}$.

High fructose diets have also been shown to cause an elevation in oxidative stress through the production of reactive oxygen species. Multiple studies have noted an 
increase in oxidative stress in rats fed a high fructose diet through observing suppressed levels of antioxidant enzymes such as gluathione and elevated levels of markers of oxidative stress, such as protein carbonylation and lipid peroxidation ${ }^{94,95}$. A study by Levi et al. suggests that high fructose diets may have a potent effect on oxidative stress, as rats fed a high fructose diet had the highest urinary lipid peroxidase output compared to rats fed both glucose and sucrose ${ }^{62}$. This may be due to formation of ROS such as hydrogen peroxide during protein glycation ${ }^{96}$.

Additionally, rats fed high fructose diets have been shown to develop insulin resistance due to the hyperglycemic conditions the diet promotes ${ }^{97}$. This diet has also been shown to cause the development of non-alcoholic fatty live disease (NAFLD) as a result of hepatic stress due to fructose metabolism, as fructose is primarily metabolized through the liver ${ }^{98}$. NAFLD is characterized by steatosis of the liver when excessive alcohol consumption is absent ${ }^{99,100}$.

\subsection{BONE QUALITY}

Initially, the ability to resist fracture was studied primarily based on bone mineral density (BMD) alone; however the concept of bone strength has changed to include many different parameters of bone biology, encompassed in one term: Bone Quality ${ }^{101}$. Bone quality is an important term that speaks to all of the properties that allow bone to resist

fracture, including structural, material, and bone remodeling aspects of the bone ${ }^{101}$. This includes but is not limited to BMD, microarchitecture, geometry, the quality of collagen, mineral size, rate of turnover and mechanical properties ${ }^{102}$. All of these parameters must be considered together to accurately assess bone quality, and therefore fragility ${ }^{101}$. 


\subsubsection{BONE MINERAL DENSITY}

BMD refers is the total density of hydroxyapatite in bone and is strongly correlated with bone strength and fragility ${ }^{103,104}$. As there are currently no dependable means to measure bone quality, skeletal mass and bone density are often used to predict fragility and diagnosis osteoporosis ${ }^{36}$. A commonly used and widely applied clinical measurement of bone quality and risk of fracture is DXA. DXA is a non-invasive measurement utilizing X-ray beams of two-peak energies. The X-ray beams are aimed at the patient's bones and their absorptions are recorded allowing for the determination of BMC normalized by the two-dimensional projected area of the bone ${ }^{105}$. While DXA has been useful and important in diagnosing this disease, it also poses several limitations. It scans the bone from an areal view providing results from a two-dimensional picture, so the size of the bone can have an effect on its apparent density while the true volumetric density of the bone is not considered ${ }^{36,105}$. Other conditions such as osteomalacia and osteoarthritis have also led to errors in the diagnosis of osteoporosis, as they can skew the DXA readings ${ }^{36}$.

A more reliable measurement of BMD comes from the use of microcomputed tomography (microCT), a non-destructive technique that allows for a three-dimensional reconstruction an object ${ }^{36,106}$. It does this through taking multiple two-dimensional x-ray images as the sample rotates. The two-dimensional projections are then reconstructed to form a three-dimensional image ${ }^{106}$. This is useful because it can measure the volumetric BMD (vBMD), which is a more accurate representation of the actual BMD of a given sample. This technology also allows the density, volume and geometry of a sample to be separated, and therefore gives more information about each sample ${ }^{36}$. 


\subsubsection{GEOMETRY AND MICROARCHETECTURE}

Bone geometry and microarchitecture refer to the spatial distribution of the bone volume, including bone size and shape ${ }^{107,108}$. These two factors are quickly becoming just as important as BMD in the determination of fracture risk. In cortical bone, the bone size and tissue distribution contribute to fracture risk ${ }^{108}$. The most studied characteristics include cross-sectional area, anterior-posterior diameter, and medial-lateral diameter, which quantify the distribution of materials and produce accurate responses to the to applied forces ${ }^{107}$.

In trabecular bone, the size, orientation and distribution of the trabeculae determine the structural contribution to the mechanical properties of the bone ${ }^{108}$. Thus, the microarchitecture is defined by trabecular number, separation, thickness and connective density, and can be a very important determinant in the evaluation of risk fracture, independently of $\mathrm{BMD}^{108}$. In a study by Legrand et al, they found that men with identical BMDs had different prevalence in fractures due to the trabecular bone microstructure, and that trabecular connectivity was more strongly correlated with multiple fractures then BMD was ${ }^{109}$. Bone geometry and microarchitecture of small animals is often observed and measured through the use of microCT technology ${ }^{106}$.

\subsubsection{BONE REMODELING}

Bone is a dynamic tissue that is subject to constant remodeling by bone cells. This remodeling is very important to the mechanical strength of the bone, repair of microfractures and calcium homeostasis ${ }^{21}$. This process is reliant on the bone cells and the balance of bone resorption and formation must be accurate to maintain a constant 
bone mass ${ }^{21}$. Bone remodeling is often assessed using undecalcified and decalcified histomorphometry analysis ${ }^{3}$, performed by histological staining of bone sections to evaluate the activity of bone cells ${ }^{110-112}$. Bone formation parameters are measured through the use of static and dynamic histomorphometry. Static histomorphometry includes stains such as Goldner's Trichrome ${ }^{110}$ which assess osteoid volume and surface, while fluorochromes such as calcein green quantify the rate of new bone formation in dynamic histomorphometry ${ }^{111}$. Bone resorption parameters are often measured using tartrate resistant acid phosphatase (TRAP) stained sections which assess the number and surface of TRAP-expressing osteoclasts ${ }^{112}$.

\subsection{BLOOD AND BONE BIOCHEMISTRY}

\subsubsection{AGE EXPRESSION}

AGEs can exist as cross-linking AGEs such as pentosidine and non-crosslinking AGEs such as CML. Pentosidine and CML are the mostly commonly measured AGEs, and are commonly used as biomarkers for the glycation of proteins ${ }^{60,63}$. As AGEs can be found bound to proteins and circulating within the blood serum, a variety of assays have been developed to measure and quantify their expression. To quantify AGE levels in biological fluids like blood serum enzyme-linked immunosorbant assays (ELISA) have been frequently used as it allows rapid detection of these protein adducts ${ }^{113}$. AGEs that are found in tissues are commonly measured through bio-analytical techniques such as high-performance liquid chromatography (HPLC). HPLC uses analytical chemistry to separate the components in a mixture allowing for the precise, rapid and reliable quantification of AGE levels ${ }^{113}$. The most common AGE measured through this analysis 
is pentosidine as it is stable under acid protein hydrolysis, naturally fluorescent, and is detectable at low concentrations ${ }^{113}$.

\subsubsection{RAGE EXPRESSION}

RAGE can be found in two forms, as a cell-membrane receptor or as a soluble isoform of the cell-membrane receptor missing the transmembrane and cytosolic domains (sRAGE) ${ }^{68}$. Immunochemical detection is often used to measure the expression of both forms of RAGE. ELISAs are commonly used to quantitatively measure sRAGE levels, as it is usually expressed in blood serum ${ }^{114}$. The use of immunohistochemistry for detection of membrane-bound RAGE is commonly used as it allows for the exact localization of the receptor in an organ or tissue. It also allows for the co-localization of AGEs and cellular markers if necessary ${ }^{113}$.

\subsubsection{OXIDATIVE STRESS EXPRESSION}

Oxidative stress, conveyed by the presence of ROS, can lead to oxidative modifications of a multitude of proteins ${ }^{115}$. One type of oxidative modification is protein carbonylation, which is an irreversible, non-enzymatic modification of proteins characterized by the addition of a carbonyl group ${ }^{116}$. It can be created through direct oxidation of proteins by ROS, as a by-product of the Maillard reaction, and through the adduction of carbonyl-containing oxidized lipids derived from the metal-catalyzed oxidation of polyunsaturated fatty acids ${ }^{116}$. As carbonyl groups are relatively stable, their

detection is often used as a standard marker of oxidative stress ${ }^{115,116}$. Carbonyl groups are 
detected and quantified through derivatization by carbonyl reagents and subsequent spectrophotometric analysis ${ }^{115}$.

\subsubsection{ENZYMATIC COLLAGEN CROSS-LINK EXPRESSION}

Bone collagen contains natural crosslinks that are formed through enzymatic reactions and are essential to the biological and biomechanical integrity of bone ${ }^{117,118}$. There are two types of predominant crosslinks within the bone; immature crosslinks including dehydro-dihydroxylysinonorleucine and dehydro-hydroxyxylysinonorleucine, and their mature counterparts, pyridinum and pyrrole cross-links ${ }^{117}$. Enzymatic action through lysine hydroxylases and lysyl oxidases facilitates enzymatic cross-link production between hydroxylysine and lysine amino acids between neighbouring molecules ${ }^{117}$. The immature cross-links occur between two molecules, and they mature into stable trivalent crosslinks, which exist between three neighbouring sites ${ }^{118}$. Pyridinolines are often used as a biomarker for bone remodeling in urine due their fluorescent properties ${ }^{118}$. These properties are equally useful to evaluate their presence within bone, both methods using HPLC for quantification ${ }^{118}$. 


\section{STUDY OBJECTIVES AND HYPOTHESES}

The current study aims to investigate if a high amount of bone loss is correlated with elevated AGE content, and whether the loss of estrogen resulting from menopause leads to an increased expression of RAGE. We hypothesize that:

1) The amount of bone lost due to ovariectomy is higher when bone AGE content is higher.

2) Ovariectomy of female rats will increase osteoclast RAGE expression.

Specifically, bone quality and AGE and RAGE expression were assessed by evaluating the following parameters:

1) Areal and volumetric bone mineral density

2) Bone geometry and microarchitecture

3) Tissue level bone remodeling

4) Serum and bone AGE content

5) RAGE expression

6) Protein carbonylation 


\section{MATERIALS AND METHODS}

\subsection{ANIMAL HUSBANDRY}

61 3-month old virgin Wistar rats were obtained from Charles Rivers Laboratories (Quebec, Canada). The rats were housed two to a cage at the Division of Comparative Medicine (DCM) at the University of Toronto. They were all housed in the same room, with consistent temperature and humidity and a light and dark cycle of 12 hours each. Rats were monitored daily by animal technicians at DCM. All cages were lined with cornmeal bedding and contained a red hide-away tube. Each cage was outfitted with a water pipe supplying an unlimited supply of reverse-osmosis water. All animal work was approved by the University Animal Care Committee at the University of Toronto and all experimental procedures were performed in accordance with the Ontario Animals for Research Act and the University of Toronto Animal Care Policies and Guidelines.

\subsection{TREATMENT}

Upon arrival to the facility the rats were randomized into six groups of ten. Three of the groups were fed a high fructose diet (HFD) containing $60 \%$ fructose by weight (Teklad Diets, Harlan Laboratories Inc) while the remaining three groups were fed a control diet (CD) of rat chow, which was isocaloric to the HFD and contained 5\% fructose (Teklad Diets, Harlan Laboratories Inc) (Table 3-1). After three months of feeding, two groups (one from the HFD group and one from the CD group) were sacrificed at 6 months of age to establish baseline parameters. The remaining four groups underwent surgical procedures. Two groups (one from CD and one from HFD) underwent 
bilateral OVX surgery. The remaining two groups underwent SHAM operations, in which their ovaries were exposed but not excised. These four surgical groups continued on a maintenance diet (Teklad Diets, Harlan Laboratories, Inc) designed to promote longevity and normal body weight in rodents without fructose or fructose substitutes. The rats continued on this diet for three months to allow the OVX groups to lose bone, and were sacrificed at 9 months of age (Figure 3-1).

\begin{tabular}{|c|c|c|}
\hline Ingredients & Control Diet $(\mathrm{g} / \mathrm{Kg})$ & High Fructose Diet $(\mathrm{g} / \mathrm{Kg})$ \\
\hline Corn starch & 340 & 600 \\
\hline Fructose & - & - \\
\hline Maltodextrin & 160 & - \\
\hline Sucrose & 100 & 207 \\
\hline Casein & 207 & 3 \\
\hline DL-Methionine & 3 & 50 \\
\hline Lard & 50 & 79.81 \\
\hline Cellulose & 79.96 & 0.04 \\
\hline Mineral Mixture & 0.04 & 10 \\
\hline Vitamin Mixture & 10 & 0.15 \\
\hline Green Food Colour & - & \\
\hline & & \\
\hline
\end{tabular}

Table 3-1. Ingredients of control and high fructose diets. 


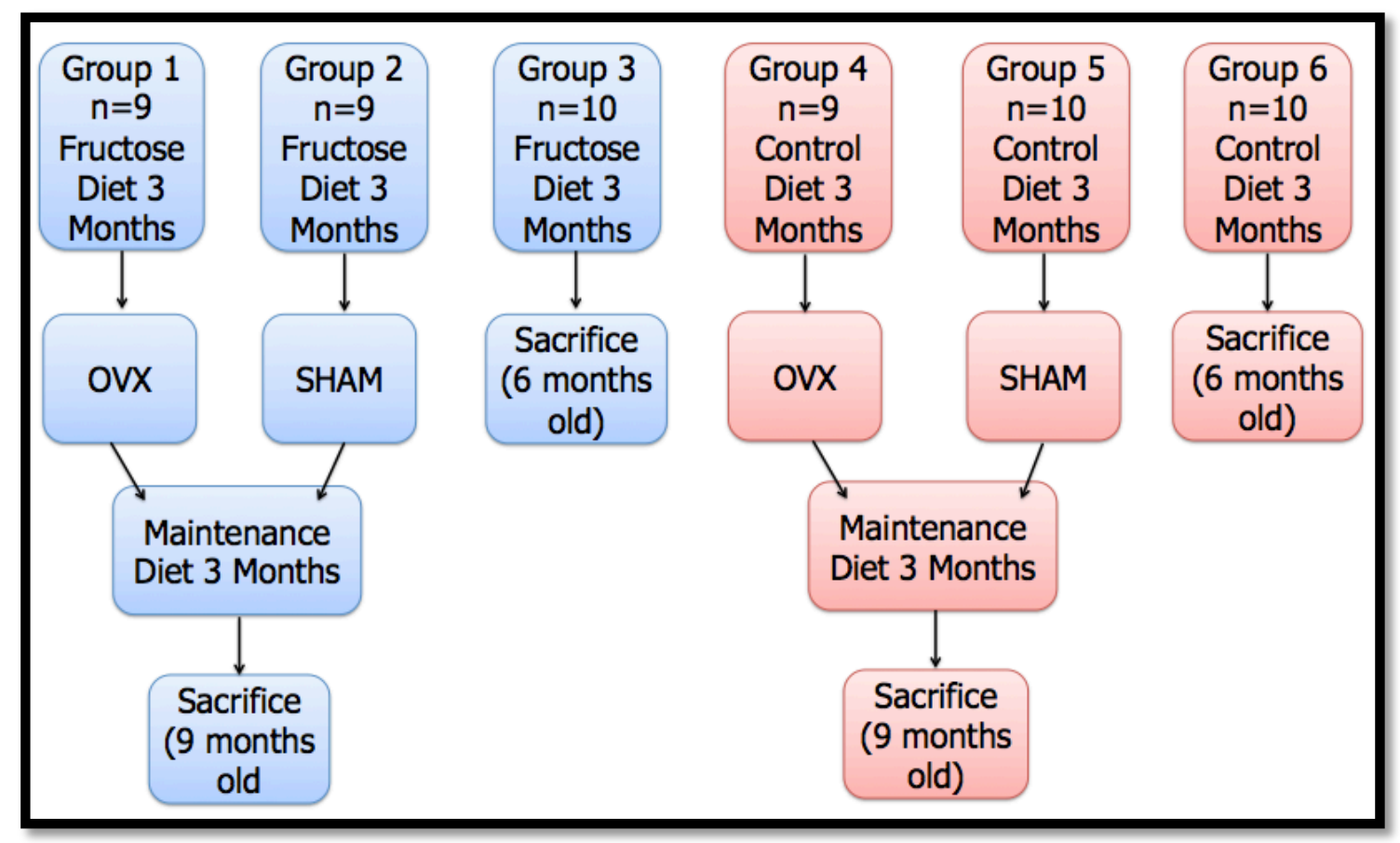

Figure 3-1. Study design

Blood was drawn from tail veins at various time-points throughout the study. The first withdraw was at the beginning of the study, before the diets were administered, the second was during the first sacrifice and surgeries, the third was four weeks after the surgical procedures, and lastly during the final sacrifice (Figure 3-2).

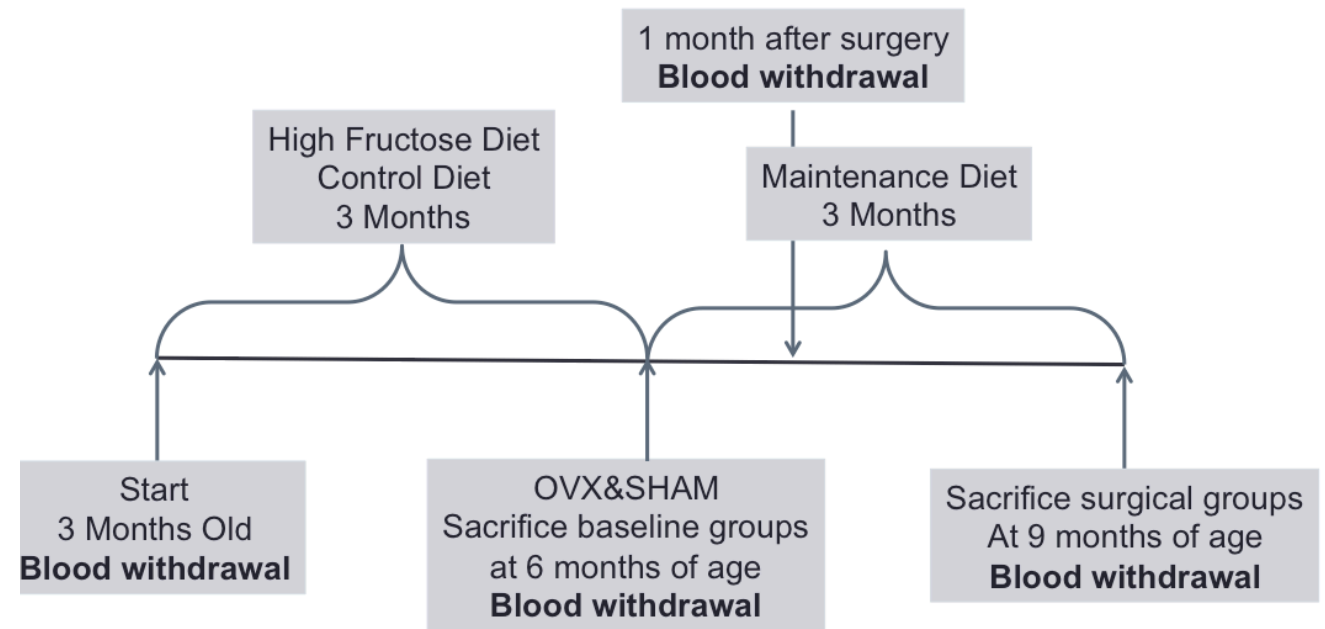

Figure 3-2. Study timeline 
There were six separate groups evaluated in this study. Two baseline groups that received the diet but had no surgical interventions: the HFD fed group (HFB- high fructose diet baseline) and the $\mathrm{CD}$ fed group (CDB- control diet baseline). Four surgical groups: the HFD fed group that underwent OVX surgery (HF+OVX), the HFD fed group that underwent SHAM surgery (HF+SHAM), the CD fed group that underwent OVX surgery $(\mathrm{CD}+\mathrm{OVX})$, and the $\mathrm{CD}$ fed group that underwent SHAM surgery (CD+SHAM).

\subsubsection{SACRIFICE AND DISSECTION}

At the end of each of their respective treatment periods, animals were placed under isoflurane anesthesia and cardiac puncture was performed to gain final blood samples followed by cervical dislocation. Immediately after sacrifice, sections of liver were removed and placed in $10 \%$ neutral buffered formalin for 2 days prior to paraffin embedding. Femurs, humeri and $1^{\text {st }}-6^{\text {th }}$ lumbar vertebrae were dissected, cleaned of soft tissue, wrapped in saline soaked gauze and stored frozen at $-20^{\circ} \mathrm{C}$. Tibias were then dissected, cleaned and cut mid shaft. Proximal regions of the right tibia were fixed in 10\% neutral buffered formalin for 5 days prior to decalcification with ethylenediaminetetraacetic acid (EDTA). Proximal regions of the left tibias were fixed in $70 \%$ ethanol for 5 days prior to Spurr resin processing.

\subsection{EVALUATION OF TREATMENT EFFECTS}

Several techniques were employed to investigate bone quality, and AGE and RAGE expression after diet and surgical interventions (Table 3-2). Specifically, DXA analysis was performed on the L6 vertebrae and left femurs to assess areal BMD (aBMD). 
Similarly, microCT analysis was conducted on the same bones to assess the vBMD and microarchitecture of these bones. Undecalcified histomorphometry analysis was done on sections of tibias embedded in Spurr resin, while decalcified histomorphometry was conducted on sections of tibias embedded in paraffin. Hematoxylin and eosin (H\&E) staining was performed on liver sections embedded in paraffin. ELISA was completed on blood serum samples to measure circulating AGEs and soluble RAGE. Protein carbonylation assays were performed on bone powder from L1-L3 to measure oxidative damage. HPLC was performed on L4 vertebrae to determine bone crosslinks. Immunohistochemistry was performed on decalcified bone sections to evaluate expression of RAGE on osteoclasts. The right femur, both humeri and L5 were stored at $20^{\circ} \mathrm{C}$ as backup bones.

\begin{tabular}{|l|l|l|}
\hline \multicolumn{1}{|c|}{ Experiment } & \multicolumn{1}{|c|}{ Specimen } & \multicolumn{1}{c|}{ Analyzed } \\
\hline DXA & L6 vertebrae, left femurs & aBMD, aBMC \\
\hline microCT & L6 vertebrae, left femurs & $\begin{array}{l}\text { vBMD, geometry and } \\
\text { architecture }\end{array}$ \\
\hline $\begin{array}{l}\text { Undecalicifed } \\
\text { histomorphometry }\end{array}$ & $\begin{array}{l}\text { Left tibias embedded in } \\
\text { Spurr resin }\end{array}$ & Tissue level bone formation \\
\hline $\begin{array}{l}\text { Decalcified } \\
\text { histomorphometry }\end{array}$ & $\begin{array}{l}\text { Right tibias embedded in } \\
\text { paraffin }\end{array}$ & Tissue level bone resorption \\
\hline H\&E & $\begin{array}{l}\text { Liver section embedded in } \\
\text { paraffin }\end{array}$ & $\begin{array}{l}\text { Steatosis, inflammation and } \\
\text { fibrosis }\end{array}$ \\
\hline ELISA & Blood serum & $\begin{array}{l}\text { Circulating AGEs and } \\
\text { soluble RAGE }\end{array}$ \\
\hline $\begin{array}{l}\text { Protein Carbonylation } \\
\text { Assay }\end{array}$ & L1-L3 vertebrae & $\begin{array}{l}\text { Bone collagen } \\
\text { carbonylation }\end{array}$ \\
\hline HPLC & L4 vertebrae & Bone collagen crosslinks \\
\hline Immunhistochemistry & $\begin{array}{l}\text { Right tibias embedded in } \\
\text { paraffin }\end{array}$ & $\begin{array}{l}\text { Osteoclast expression of } \\
\text { RAGE }\end{array}$ \\
\hline
\end{tabular}

Table 3-2. List of techniques employed bones used for each technique and parameters analyzed. 


\subsubsection{DUAL-ENERGY X-RAY ABSORPTIOMETRY (DXA)}

The L6 vertebrae and left femurs were thawed at $4^{\circ} \mathrm{C}$ overnight and DXA analysis was performed using a small animal PIXImus densitometer (Lunar; GE Corp, Mississauga, Ontario). To calibrate the PIXImus, a phantom was scanned prior to each session of scanning. During each scan, bone samples were positioned on a polystyrene plate in the same orientation for consistency in measurements. For the femurs, the region of interest was kept consistent as a rectangular box that measured 100 by 270 pixels. For the vertebrae, the region of interest was kept consistent as a box that measured 100 by 110 pixels. Results were then analyzed using the PIXImus software to determine BMC and bone area, and to calculate the $\mathrm{aBMD}(\mathrm{aBMD}=\mathrm{BMC} /$ bone area $)$.

\subsubsection{X-RAY MICROCOMPUTED TOMOGRAPHY (MICROCT)}

The $\mathrm{L} 6$ vertebrae and left femurs were thawed at $4^{\circ} \mathrm{C}$ overnight and scanned using the Skyscan 1174 Compact Desktop MicroCT Machine (Bruker-MicroCT, Belgium). After thawing the samples were wrapped tightly in two layers of saline soaked gauze and placed inside a $10 \mathrm{~mL}$ microtube. Care was taken to ensure that both the femurs and vertebrae were positioned vertically within the tubes, and the microtubes were secured to the scanning platform using double-sided tape (Figure 3-3). The following setting were used for the scans: $\mathrm{x}$-ray voltage $=50 \mathrm{kV}$, $\mathrm{x}$-ray current $=800 \mu \mathrm{A}$, frame averaging $=2$. A $0.25 \mathrm{~mm}$ aluminum filter was applied and an isotropic voxel size of $11.6 \mu \mathrm{m}$ was used for both femurs and vertebrae. At the end of each day hydroxyapatite phantoms of 750 and $1300 \mathrm{mgHA} / \mathrm{cm}^{3}$ were scanned for calibration of BMD measurements. 
All femurs were scanned in a vertical orientation, with the head of the femur directly adjacent to the scanning platform. Since the femurs were too large to be completely included in the 11.6-voxel scanning-field of view, they were scanned at the mid-diaphysis. To accurately locate the mid-diaphysis, femurs were measured before being scanned with a pair of standard calipers and an oil-free Fimo polymer clay (Fimo Classic, Eberhard, Farber) was wrapped around an area approximately $5 \mathrm{~mm}$ distal to the mid-diaphysis. This allowed a landmark to ensure the mid-diaphysis was measured in the scans (Figure 3-3B).

A

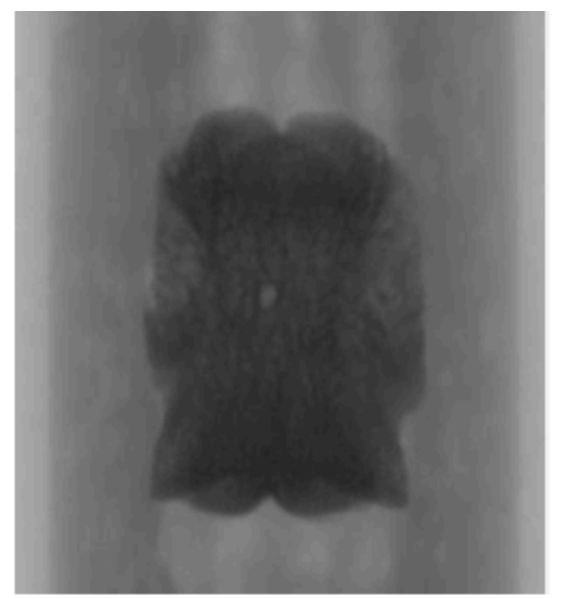

B

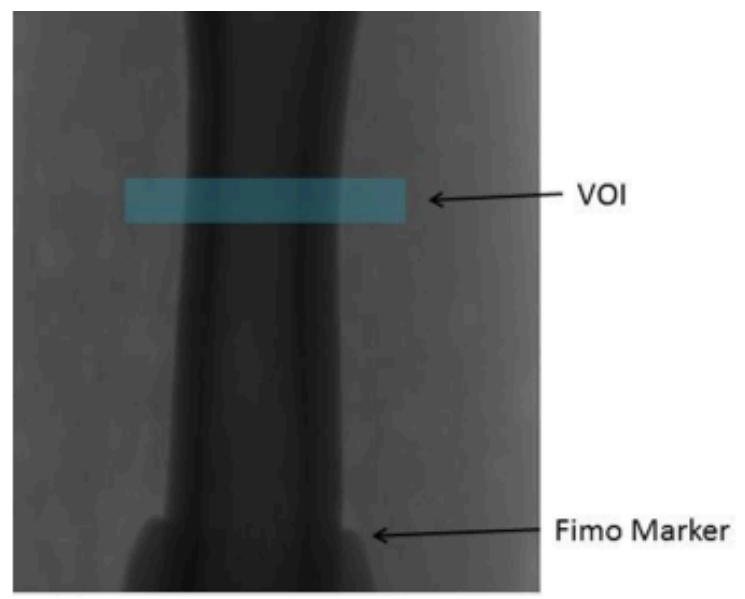

Figure 3-3. MicroCT scanning field of view at $11.6 \mu \mathrm{m}$ voxel size. (A) representative image of a L6 vertebrae (B) representative image of a femur. The blue box denotes the 1 $\mathrm{mm}$ volume of interest at the mid-diaphysis used for microCT analysis.

Following scanning, three-dimensional images of each sample made through reconstruction using Skyscan NRecon software (Version 1.6.3.2) were analyzed using Skyscan CTan software (Version 1.10.0.1). All scans were reconstructed using the following settings: smoothing $=1$, ring reduction $=6$, beam hardening correction $=30 \%$, defective pixel masking $=50 \%$.

For femoral scans, the mid-diaphysis was located through use of the Fimo landmark, and a $1 \mathrm{~mm}$ thick volume of interest (VOI) for analysis was chosen to be 0.5 
$\mathrm{mm}$ above and below the mid-diaphysis. Analysis of the VOI was done to obtain a number of parameters including anterior-posterior diameter (A-P diameter), medial-lateral diameter (M-L diameter), cross-sectional bone area, cortical thickness, cortical porosity, and cortical vBMD. For cross-sectional bone area, A-P and M-L diameter the region of interest (ROI) was the whole femoral cross section (Figure 3-4A). The ROI used to calculate the vBMD and cortical thickness encompassed the cortex only (Figure 3-4B).

A

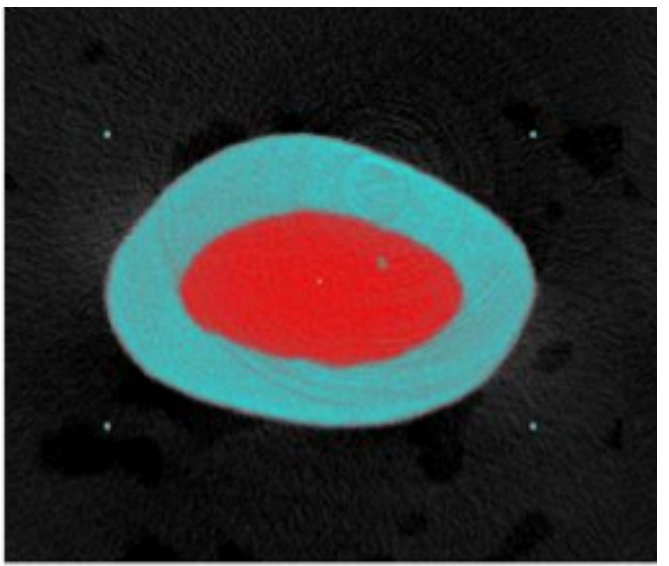

B

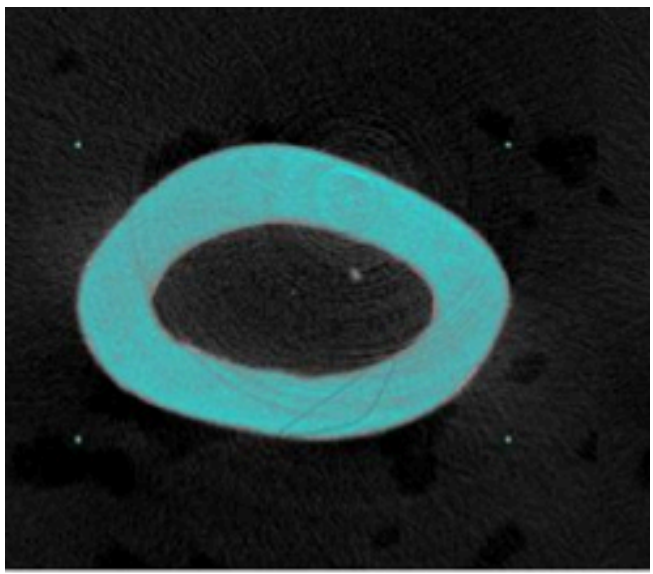

Figure 3-4. ROIs used for microCT analysis of cortical bone. Bone included in analysis is highlighted in blue, while marrow included in analysis is highlighted in red. (A) representative image of a ROI for whole femoral cross section (B) representative image of a ROI for femoral cortex.

For vertebral scans, portions of the bone that contained primary spongiosa near the growth plates were excluded, leaving only secondary spongiosa to be analyzed. Parameters including percent bone volume (BV/TV), trabecular vBMD, tissue vBMD, trabecular number, trabecular thickness, and trabecular separation were determined from microCT analysis. For tissue vBMD, whole vertebral cross-sections were used as the ROI (Figure 3-5A). For all other parameters, ROIs that contained only the trabecular bone were drawn for each individual sample (Figure 3-5B). 

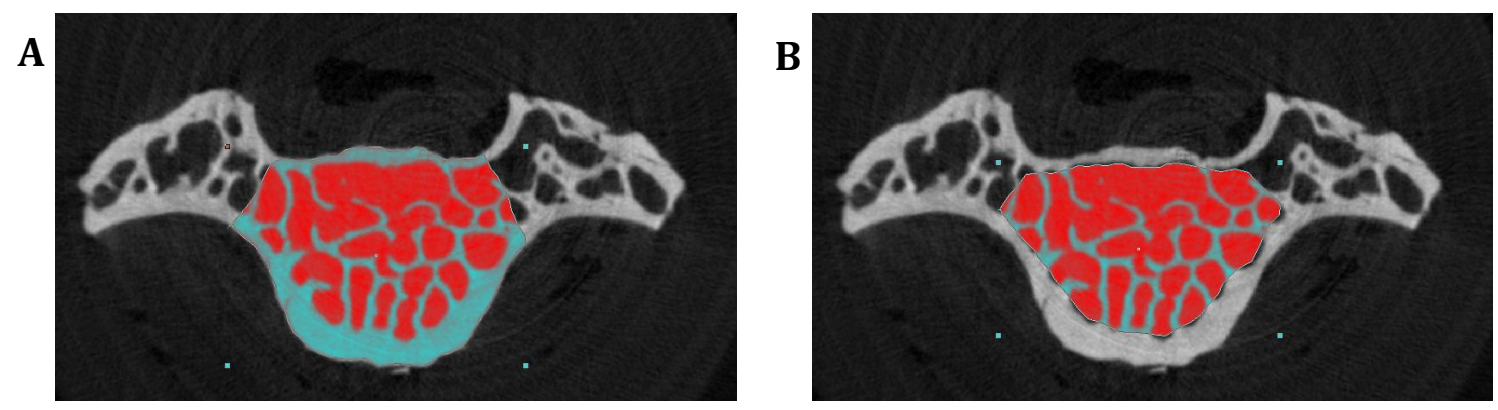

Figure 3-5. ROIs used for microCT analysis of trabecular bone. Bone included in analysis is highlighted in blue, while marrow included in analysis is highlighted in red. (A) representative image of a ROI for whole vertebral cross section. (B) representative image of a ROI for trabecular bone.

\subsubsection{UNDECALCIFIED HISTOMORPHOMETRY}

Undecalcified bone histomorphometry was used to evaluate tissue level bone formation parameters. The rats received $10 \mathrm{mg} / \mathrm{kg}$ calcein green (Sigma-Aldrick, Oakville, ON) injections subcutaneously three and ten days before being sacrificed. The left tibias were dissected, cleaned of soft tissue and fixed for 5 days in $70 \%$ ethanol. They were then dehydrated in ascending concentration of acetone, and infiltrated with Spurr resin under vacuum. They were then embedded in Spurr resin and five sequential seven $\mu \mathrm{m}$ coronal sections were cut using a Leica RM2265 rotary microtome (Leica, Wetzlar, Germany). The sections were mounted onto gelatinized slides and incubated in a $60{ }^{\circ} \mathrm{C}$ oven for 48 hours to ensure proper adherence of the sections.

\subsubsection{GOLDNER'S TRICHROME STAIN}

One of the seven $\mu \mathrm{m}$ sections was stained with Goldner's Trichrome stain. First the bones were rehydrated in descending concentrations of ethanol and then stained by sequential incubations. The procession of staining went as follows; 15 minute incubation 
in Weigert's Iron Hematoxylin, 15 minutes in Ponceau Acid Fuchsin, 8 minutes in Phosphomolybdic Acid/Orange G and 15 minutes in Light Green. Mineralized bone is stained green, osteoid is stained red and cell nuclei are stained dark brown to black (Figure 3-6) ${ }^{119}$. Osteoid is the organic matrix that is secreted by mature osteoblasts that becomes mineralized into new bone. Goldner's stain is used to measure the osteoid seam, which provides information about tissue-level bone formation. After staining, sections were cover-slipped using Permount mounting medium ${ }^{119}$.

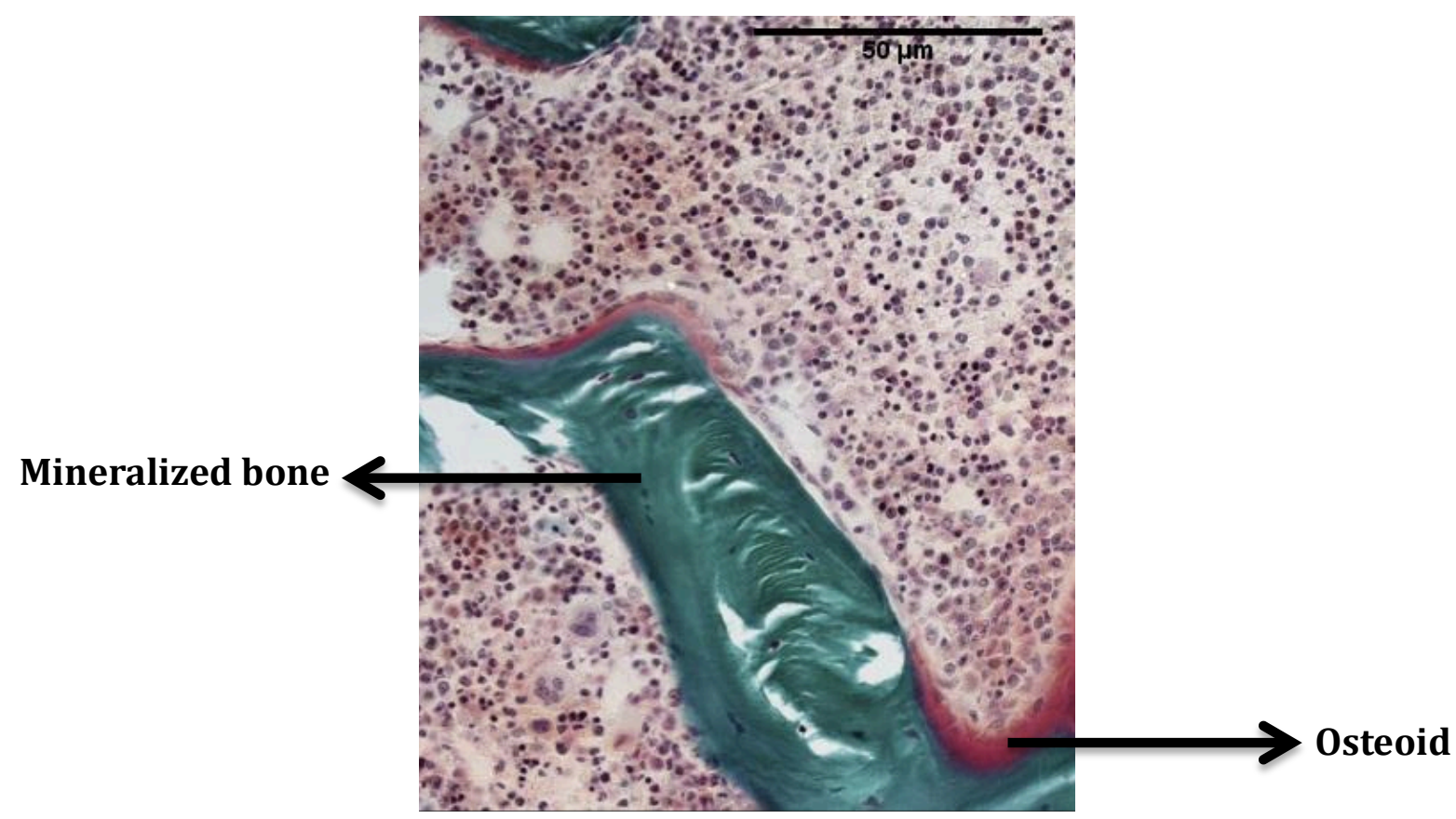

Figure 3-6. Representative image of bone section stained with Goldner's Trichrome. Image scanned at $200 \mathrm{X}$ magnification.

The slides were viewed and scanned under a light microscope at 200X magnification, and the results were quantified using the Bioquant Osteo 11.2.6 MIR software (Bioquant Image Analysis Corporation, Nashville, Tennessee, USA). The trabecular bone of the secondary spongiosa in the proximal tibial metaphysis was analyzed. The ROI began 0.5 
$\mathrm{mm}$ from the distal end of the growth plate, extending $2 \mathrm{~mm}$ into metaphysis, and $0.5 \mathrm{~mm}$ from the endosteal surface (Figure 3-7). This was done to ensure the primary spongiosa near the growth plate was excluded, as it is not representative of metaphyseal trabecular bone ${ }^{87,120,121}$. The bone formation parameters measured were osteoid surface (OS, mm) and osteoid volume $\left(\mathrm{OV}, \mathrm{mm}^{2}\right)$. These are primary measurements of the osteoid, which are normalized over total bone surface and total bone volume to give percent osteoid surface (OS/BS, \%) and percent osteoid volume (OV/BV,\%), respectively. Finally osteoid width $(\mathrm{O} . \mathrm{Wi}, \mu \mathrm{m})$ is measured to obtain the thickness of the osteoid seam ${ }^{122}$.

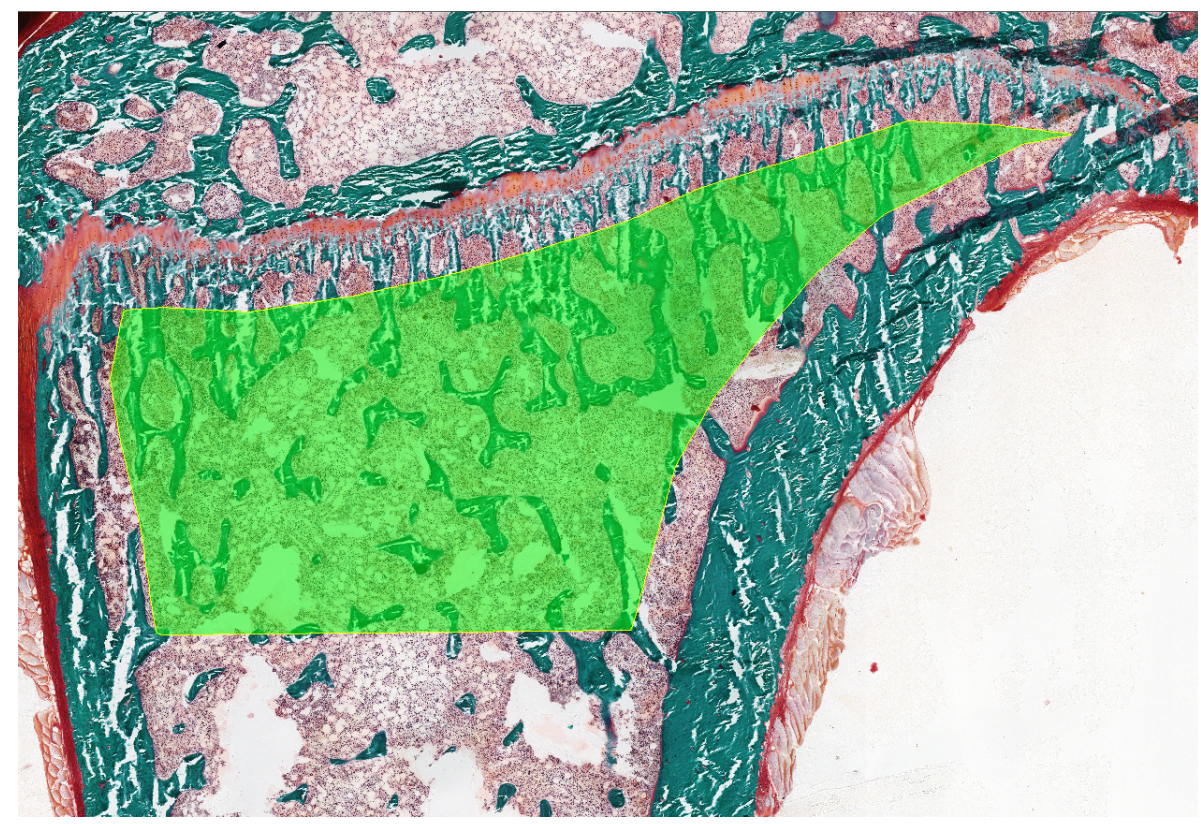

Figure 3-7. Representative image of the region of interest for analysis of Goldner's Trichrome stained sections.

\subsubsection{CALCEIN GREEN LABELING}

Calcein green is a fluorochrome that strongly chelates to calcium ions and thus is incorporated into bone during mineralization. These molecules fluoresce under specific 
wavelengths, and can be viewed under ultraviolet (UV) light to detect bone surfaces that have undergone mineralization. When viewed under UV light calcein green fluoresces bright green ${ }^{123,124}$. It is often used as a surrogate measurement for bone formation ${ }^{125}$.

Since calcein green was injected at a 7-day interval prior to sacrifice, there was opportunity for two different fluorescent labels to form over this time period. Under UV light, single labels (one line of fluorescence) along the bone surface indicate only one dose was incorporated into the bone. Double labels (two lines of fluorescence parallel to each other) along the bone surface indicate that both doses were incorporated at that location (Figure 3-8). Double labeling allows the rate of bone formation to be determined by measuring the distance between the double labels (interlabel width) and then dividing by the time interval ( 7 days).

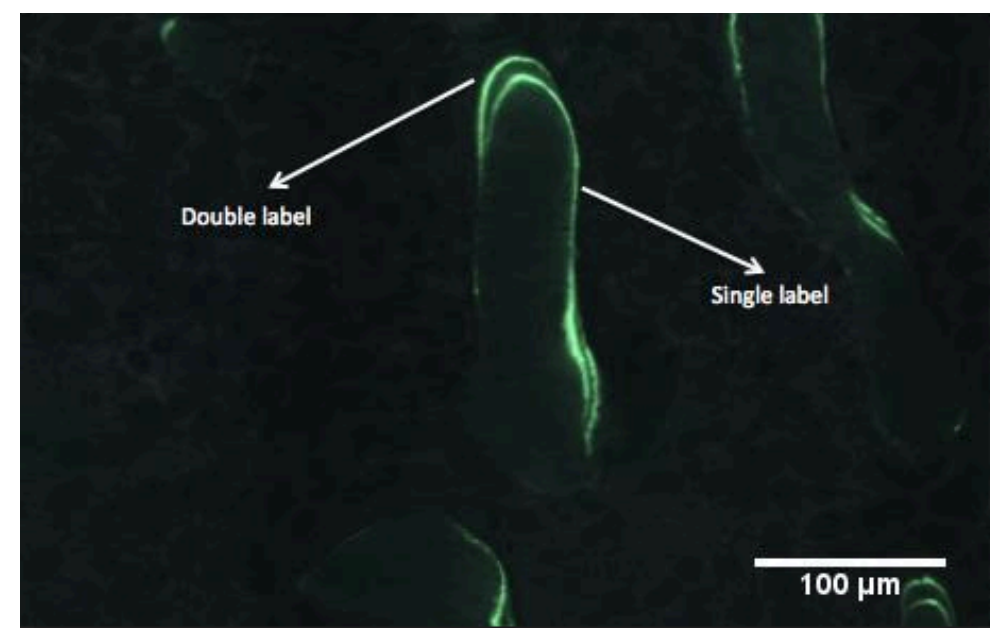

Figure 3-8. Representative image of calcein green labeling. Image scanned at 100X magnification

For quantification of calcein labels, a seven $\mu \mathrm{m}$ section, sequential to that used in the Goldner's Trichome stain was analyzed. The slide was cleared using xylene and was cover-slipped with Permount mounting medium, unstained. The sections were then viewed and scanned at $100 \mathrm{X}$ magnification. Dynamic histomorphometric analysis of 
these slides was performed using the Bioquant Osteo 11.2.6 MIR software (Bioquant Image Analysis Corporation). The region of interest was identical to the ROI used in Goldener's Trichrome analysis. The calcein lines were quantified to obtain the following unnormalized parameters: single label surface (sLS, mm); double label surface (dLS, $\mathrm{mm}$ ); mineralizing surface (MS, mm); and interlabel width (Ir.L.Width, $\mu \mathrm{m})$. Normalized parameters obtained were: percent mineralizing surface (MS/BS, \%); mineral apposition rate (MAR, um/day, formula=Ir.L.Width/labeling period); bone formation rate normalized over bone surface $(\mathrm{BFR} / \mathrm{BS}, \mu \mathrm{m} /$ day/mm, formula=MS*MAR/BS); and bone

formation rate normalized over bone volume $\left(\mathrm{BFR} / \mathrm{BV}, \mu \mathrm{m} / \mathrm{day} / \mathrm{mm}^{2}\right.$, formula= $\left.\mathrm{MS}^{*} \mathrm{MAR} / \mathrm{BV}\right)^{122}$.

\subsubsection{DECALCIFIED HISTOMORPHOMETRY}

Decalcified bone sections were used to evaluate tissue level bone resorption parameters using tartrate resistant acid phosphate (TRAP) staining. TRAP is an enzyme expressed by active osteoclasts and its expression is strongly correlated to the resorptive activity of osteoclasts ${ }^{126}$. Therefore, TRAP is commonly used as both an osteoclastic marker and an analysis tool to determine tissue level bone resorption.

After sacrifice, right tibias were dissected, cleaned of soft tissue and fixed in $10 \%$ neutral buffered formalin for 5 days. Tibias were then decalcified in $0.5 \mathrm{M}$ EDTA $(\mathrm{pH}$ 7.4) for 6 weeks at room temperature. Specimen were dehydrated through ascending concentration of ethanol and infiltrated with paraffin. The bones were then embedded within paraffin blocks and five $\mu \mathrm{m}$ thick coronal sections were cut with a Leica Reichert June 2030 microtome (Leica Microsystems Canada Inc., Richmond Hill, Ontario). The 
sections were placed on Superfrost Plus positively charged slides, and baked in a $60^{\circ} \mathrm{C}$ oven for 48 hours to ensure proper adherence. Sections were then stained for TRAP using the Acid Phosphatase Leukocyte Kit (Sigma-Aldrich) and counterstained with Acid Hematoxylin for nuclei. The sections were then coverslipped using Immunomount aqueous mounting medium.

In this stain, active TRAP-expressing osteoclasts appear red while nuclei are stained dark purple (Figure 3-9). Sections were viewed and scanned under a light microscope at 200X magnification. Analysis was performed using the Bioquant Osteo 11.2.6 MIR software (Bioquant Image Analysis Corporation). Only cells that followed the following criteria were counted: 1) had red coloring 2) had 2 or more visible nuclei 3) one edge was directly adjacent to a bone surface. The region of interest was kept identical to Goldener's Trichrome analysis. Parameters measured include normalized osteoclast number (N.Oc/BS, 1/mm) and percent osteoclast surface (Oc.S/BS, \%) ${ }^{122}$.

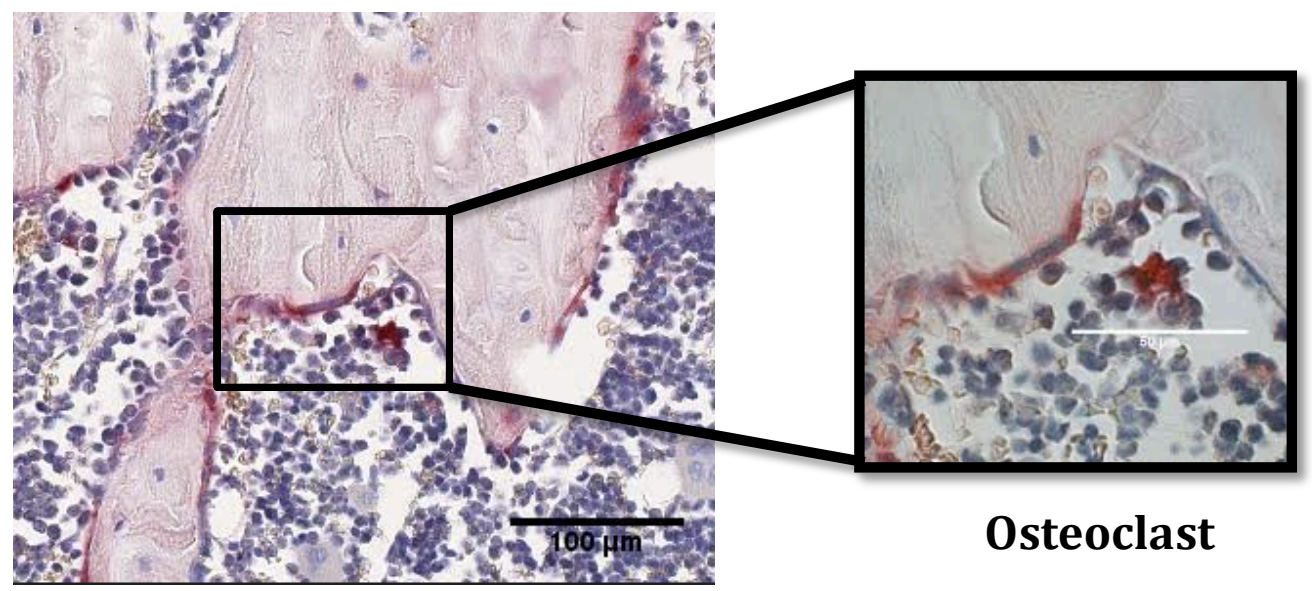

Figure 3-9. Representative image of bone section stained with TRAP. Large image scanned at 200X magnification, small image scanned at 400X magnification. 


\subsubsection{HEMATOXYLIN AND EOSIN STAINING}

As the high fructose diet has been previously shown to induce non-alcoholic fatty liver disease (NAFLD) in rats ${ }^{99,100,127}$ we examined if this occurrence was observed in our study. NAFLD is characterized through evaluation of hepatic steatosis, inflammation and fibrosis in the absence of significant alcohol use or other known liver disease ${ }^{128,129}$.

After dissection, sections of the liver were excised and placed in $10 \%$ nonbuffered formalin for 2 days. Specimen were then dehydrated through ascending concentration of ethanol and infiltrated with paraffin. The liver sections were then embedded within paraffin blocks and five $\mu \mathrm{m}$ thick sections were cut with a Leica Reichert June 2030 microtome (Leica Microsystems Canada Inc., Richmond Hill, Ontario). The sections were placed on Superfrost Plus positively charged slides, and baked in a $60^{\circ} \mathrm{C}$ oven for 48 hours to ensure proper adherence. The sections were then stained in Mayer's hematoxylin for one minute and eosin for 30 seconds, then coverslipped with Permount mounting medium. The sections were then scanned at 200X magnification under a light microscope and qualitatively assessed for steatosis, inflammation and fibrosis.

\subsubsection{ENZYME-LINKED IMMUNOSORBENT ASSAY (ELISA)}

Blood was drawn from tail veins at various times throughout the study, and through cardiac puncture during sacrifices. Blood was collected and centrifuged to separate blood plasma from the serum, which was collected and stored at $-20^{\circ} \mathrm{C}$. Levels of soluble pentosidine (USBiological, 027322), soluble CML (Cell BioLabs, STA-316) and soluble RAGE (Abcam, ab100780) expression were detected through use of anti-rat 
ELISA kits. Each of these assays utilized the competitive inhibition enzyme immunoassay technique. $100 \mu \mathrm{L}$ of standard or serum sample is added to a microplate that is pre-coated with a primary capture antibody specific for each epitope and was incubated for 2.5 hours at room temperature with gentle shaking. This was followed by 4 washes using supplied wash solutions. $100 \mu \mathrm{L}$ of the detection antibody specific for each assay was added and incubated for 1 hour at room temperature with gentle shaking, followed by 4 washes. $100 \mu \mathrm{L}$ of HRP-Streptavidin was added to each well and incubated for 45 minutes at room temperature with gentle shaking followed by 4 washes. $100 \mu \mathrm{L}$ of tetramethylbenzidine substrate solution was added to each well and incubated at room temperature in the dark. $50 \mu \mathrm{L}$ of stop solution was then added to each well and the absorbance was immediately read at $450 \mathrm{~nm}$ using a PerkinElmer EnVision 2103 Multilabel Reader. Expression of each factor was determined through comparisons to standard curves generated for each assay performed.

\subsubsection{PROTEIN CARBONYLATION ASSAY}

Carbonylation of bone collagen was investigated as a marker for oxidative damage of collagen molecules ${ }^{116}$. Demineralized bone powder from the L1-L3 vertebrae was derived with a 2,4,-dinitrophenylhydrazine (DNPH) solution and centrifuged. The resulting pellet (P1) was kept for subsequent analysis, while the supernatant (S1) was

further precipitated with $10 \%$ trichloroacetic acid. This solution was centrifuged and the supernatant (S2) and pellet (P1) were kept. Both pellets were washed twice in cold acetone, allowed to dry then were re-suspended in $6.0 \mathrm{M}$ guanidine $\mathrm{HCl} / 0.5 \mathrm{M} \mathrm{KCl} .200$ $\mu 1$ of P1, P2 and S2 were pipetted onto a microtiter plate, along with DNPH and non- 
DNPH controls in duplicate and the absorption was measured on a PerkinElmer EnVision 2103 Multilabel Reader at $405 \mathrm{~nm}$. Carbonyl content of insoluble collagen (P1) and total soluble collagen (P2 and S2) was determined by comparison to glyceraldehyde controls.

\subsubsection{HIGH-PERFORMANCE LIQUID CHROMATOGRAPHY (HPLC)}

Bone collagen crosslinks pentosidine, dioxypyridinoline and pyridinoline, as well as hydroxyproline were measured using an HPLC analysis previously published by our $1 \mathrm{ab}{ }^{130}$. The $\mathrm{L} 4$ vertebrae were hydrolyzed in $6 \mathrm{~N} \mathrm{HCl}$ at $110^{\circ} \mathrm{C}$ for 18 hours and added to a sample buffer containing $10 \%$ acetonitrile, $1 \%$ HFBA and water plus an internal standard (pyridoxine). Crosslinks were quantified using a modification of published methods by Bank et al. ${ }^{131}$ using standards of pentosidine and lysyl-pyridinoline (PolyPeptide Group, Strasbourg, France.) Hydroxyproline was quantified using a slight modification of a published method ${ }^{132}$ using both hydroxyproline and amino acid standards (Sigma-Aldrich). The sample buffers were run through Agilent Zorbax Eclipse XDB-C18 Reversed-Phase C18 HPLC columns $(150 \times 4.6 \mathrm{~mm}, 5 \mu \mathrm{m}$ particle size, $80 \AA$ pore size, end-capped; Agilent Technologies, Mississauga, ON, Canada). Elution profiles (fluorescence vs. elution time) were recorded and areas under the peaks were obtained and compared to a standard curve to measure the concentration of the crosslinks. The crosslink concentrations were normalized to the amount of collagen in the sample, estimated by hydroxyproline measurements. 


\subsubsection{IMMUNOHISTOCHEMISTRY}

The decalcified tibia sections cut when preparing for TRAP analysis were used in this assay. Four five $\mu \mathrm{m}$ sequential sections were evaluated, one was stained with TRAP to identify active osteoclasts, one was stained immunohistochemically for RAGE expression and the remaining slides were used as controls. It was important that the TRAP and immunohistochemistry stained sections were sequential to adequately examine if osteoclasts expressed on one slide were the same cells expressed on the subsequent slide. This ensures the ability to co-localize active osteoclasts with RAGE expression.

Immunohistochemistry was performed to detect RAGE using rabbit anti-RAGE antibody (ab3611, Abcam) and a staining kit (Rabbit specific HRP/DAB (ABC) Detection IHC Kit, Abcam). Sections were deparaffinised and rehydrated in decreasing concentrations of ethanol. Endogenous peroxide activity was blocked using the Hydrogen Peroxide Block for 10 minutes followed by two washes in TBS buffer. Antigen retrieval was performed through use of pepsin applied to the sections for 15 minutes at $37^{\circ} \mathrm{C}$ followed by cooling for 10 minutes at room temperature, and then the sections were washed three times. Non-specific binding was blocked by applying the Protein Block for 10 minutes followed by one TBS wash. The primary antibody (1:1000) diluted in TBS buffer with $0.4 \%$ Triton-X was applied and left overnight in a humidified chamber at $4{ }^{\circ} \mathrm{C}$. The samples were washed 4 times and incubated with the Biotinylated Goat Anti-Rabbit secondary antibody for 10 minutes followed by four washes. Streptavidin Peroxidase was applied for 10 minutes, and sections were washed four times. The expression was 
detected using a DAB chromagen applied for 3 minutes, followed by 4 washes and a counterstain with Myer's hematoxylin at a 1:3 dilution with distilled water. The sections were then coverslipped using Permount mounting medium and used for analysis. The first control was a negative control performed by omitting the primary antibody and incubating in TBS-Triton-X buffer overnight, all other steps remained consistent as above. The second control made use of an isotype control; instead of incubating with a primary antibody, the sections were incubated with a rabbit $\operatorname{IgG}$, polyclonal isotype control (Abcam, Ab 27478) at a 1:1000 dilution. The control slides were used to detect any non-specific staining by the primary and secondary antibodies. TRAP staining was done as explained previously.

The TRAP and immunohistochemistry sections were scanned at 200X magnification and were analyzed using the Bioquant Osteo 11.2.6 MIR software (Bioquant Image Analysis Corporation). TRAP analysis was performed as mentioned before, however throughout the analysis, the sequential immunohistochemical stain was cross-examined to observe if osteoclasts that stained positive for TRAP also expressed RAGE (Figure 3-10) The following parameters were measured: number of osteoclasts (N.Oc, \#), number of osteoclasts expressing RAGE (Oc.RAGE, \#), number of osteoclasts expressing RAGE over bone surface (Oc.RAGE/BS, 1/mm), and percent osteoclasts expressing RAGE over total number of osteoclasts (Oc.RAGE/N.Oc, \%) 

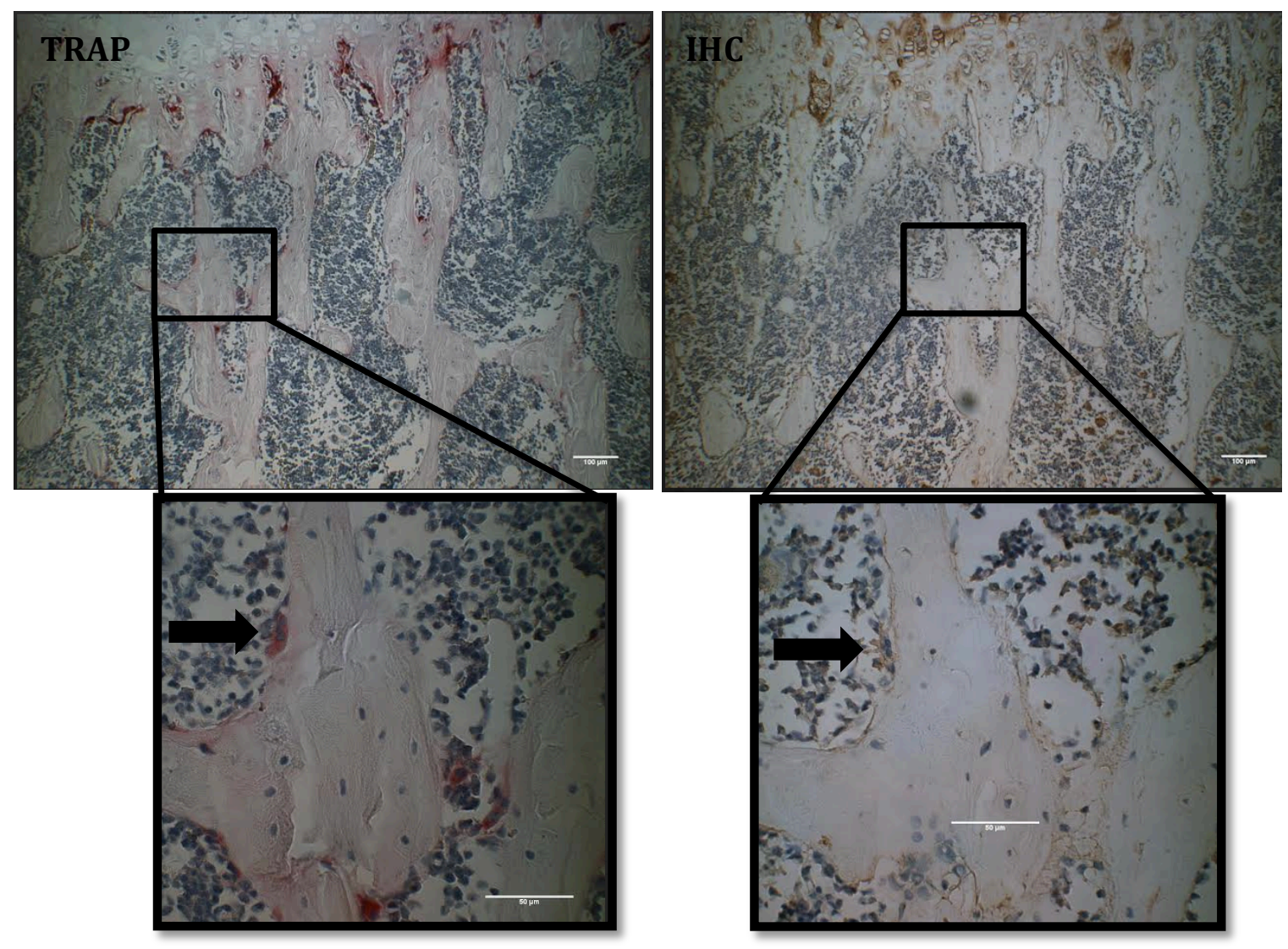

Figure 3-10. Representative images of sequential sections stained with TRAP (left) and immunohistochemistry for RAGE (right). Osteoclasts that stained positive for TRAP were cross-referenced with the IHC stained sections to examine whether the cells also expressed RAGE. Arrow signifies osteoclast co-expressing RAGE and TRAP.

\subsection{STATISTICAL ANALYSIS}

All statistical analyses were performed using SPSS statistical software (Version 21, IBM). Results between the baseline groups (HFB and CDB) were compared using an independent Student's T-Test. Results between the surgical groups were compared using two-way analysis of variance (Two-way ANOVA). A Two-way ANOVA is used to examine the influence of two different categorical independent variables on one continuous dependent variable. In this study the two categorical independent variables are 
diet, which encompasses the HFD and CD; and surgery, which encompasses SHAM and OVX. If both of the categorical variables have an effect on the dependent variable, there will be statistical significance for the interaction. Significance was defined as $\mathrm{p}<0.05$ for two-tailed probability at $95 \%$ confidence. Data are presented as mean \pm standard deviation (SD). 


\section{RESULTS}

\subsection{ANIMAL WEIGHTS}

Animals were weighed throughout the study using a tabletop scale. Rats in each surgical group gained weight similarly until after the surgical procedures, where groups that received the OVX surgery gained more weight then rats in the SHAM groups (Figure $4-1)$.

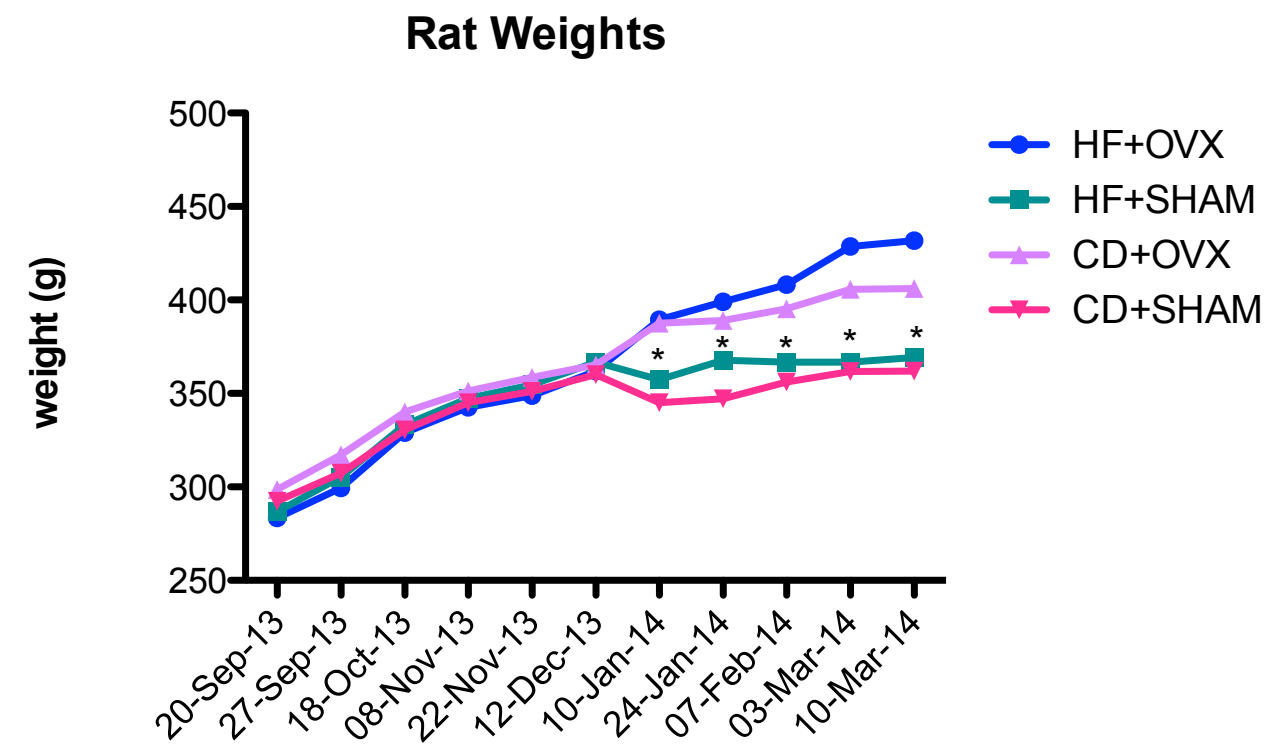

Figure 4-1: Animal weights throughout the study. At each time point weights were analyzed using a two-way ANOVA. *SHAM groups significantly different from OVX groups, $p<0.05$ for surgery effects as indicated, $p>0.05$ for diet and interaction at each time point.

\subsection{DXA ANALYSIS}

DXA was used to evaluate the BMD of the L6 vertebrae and femur. The areal $\mathrm{BMD}\left(\mathrm{aBMD}, \mathrm{g} / \mathrm{cm}^{3}\right)$ and bone mineral content $(\mathrm{BMC}, \mathrm{g})$ were measured and compared 
between treatment groups. The BMC measures the amount of bone mineral, and is normalized over the bone area to obtain the aBMD. In both the L6 vertebrae and femur of the baseline groups, the aBMD and BMC were unchanged (Figure 4-2).

A

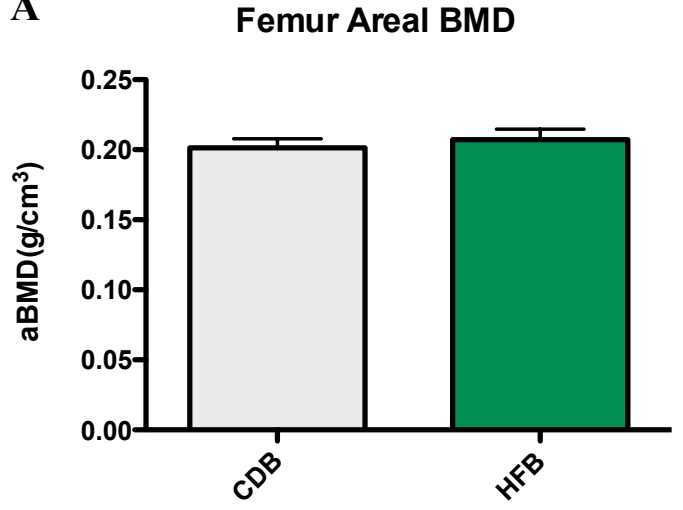

C

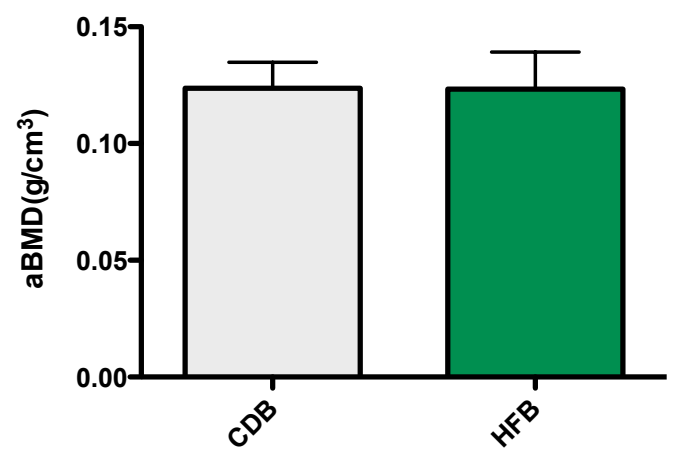

B Femur BMC

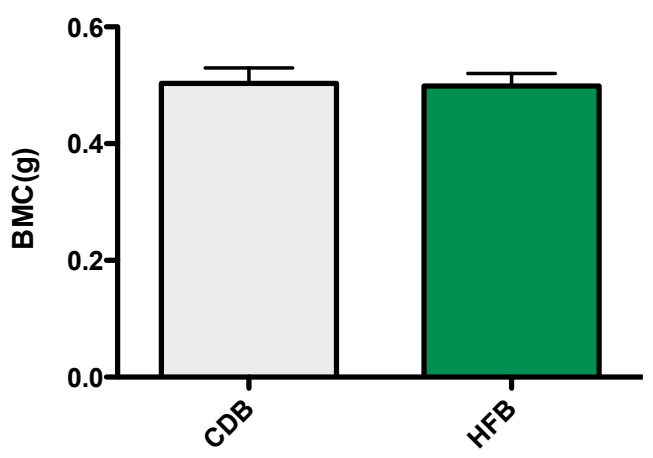

D

L6 BMC

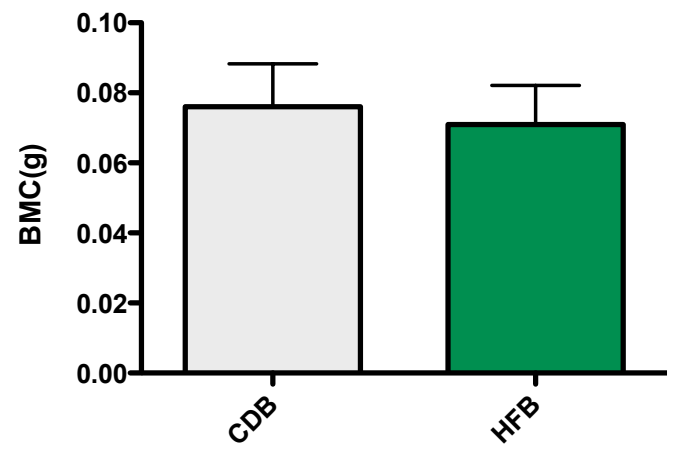

Figure 4-2. Results from DXA analysis of L6 vertebrae and femurs of baseline groups. (A) femur aBMD (B) femur BMC (C) L6 aBMD (D) L6 BMC. Student's T-test, mean \pm $\mathrm{SD}, \mathrm{n}=10$.

Analysis of the surgical groups' femurs showed there was a significant interaction between the effects of the diet and surgery on the aBMD. A two-way ANOVA showed that SHAM-operated rats had a greater aBMD, and the interaction of the CD and SHAM surgery had a significant effect on aBMD. There were no differences in interaction, diet, or surgery in the BMC (Figure 4-3). 
A Femur Areal BMD

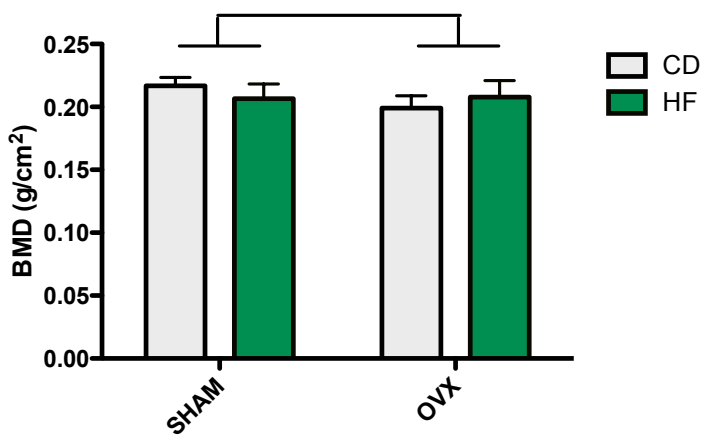

B

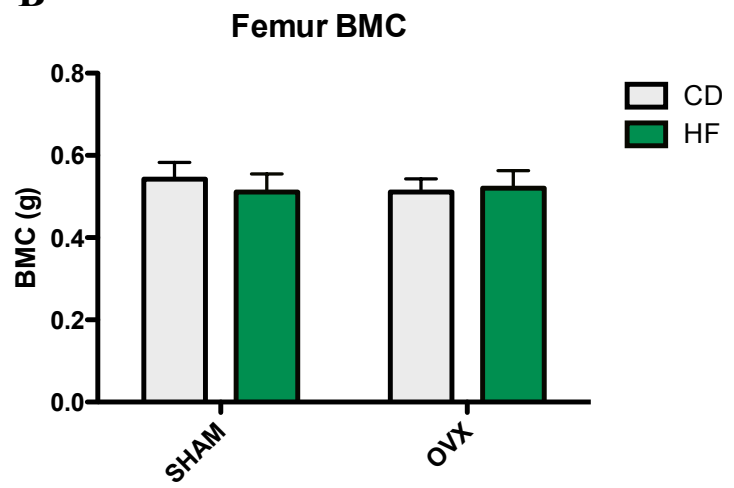

Figure 4-3. Results from DXA analysis of the femurs from the surgical groups. (A) femur BMD (B) femur BMC. Two-way ANOVA, mean $\pm \mathrm{SD}, \mathrm{n}=9$, * SHAM significantly different from OVX surgical groups. (A) diet alone $p=0.8249$, surgery alone $p=0.0218$, interaction $\mathrm{p}=0.0095$. (B) diet alone $\mathrm{p}=0.2295$, surgery alone $\mathrm{p}=0.1983$, interaction $\mathrm{p}=0.6091$.

Analysis of the L6 vertebrae from the surgical groups showed that groups that underwent SHAM surgery had a significantly higher aBMD, but there was no difference based on diet, or an interaction of surgery and diet (Figure 4-4). A two-way ANOVA showed there was not a detectable interaction between the effects of the diet and surgery, although the $\mathrm{CD}+\mathrm{SHAM}$ group did trend toward having the highest $\mathrm{BMC}(\mathrm{p}=0.06)$. The diet and surgery alone did not have a detectable effect on BMC. 

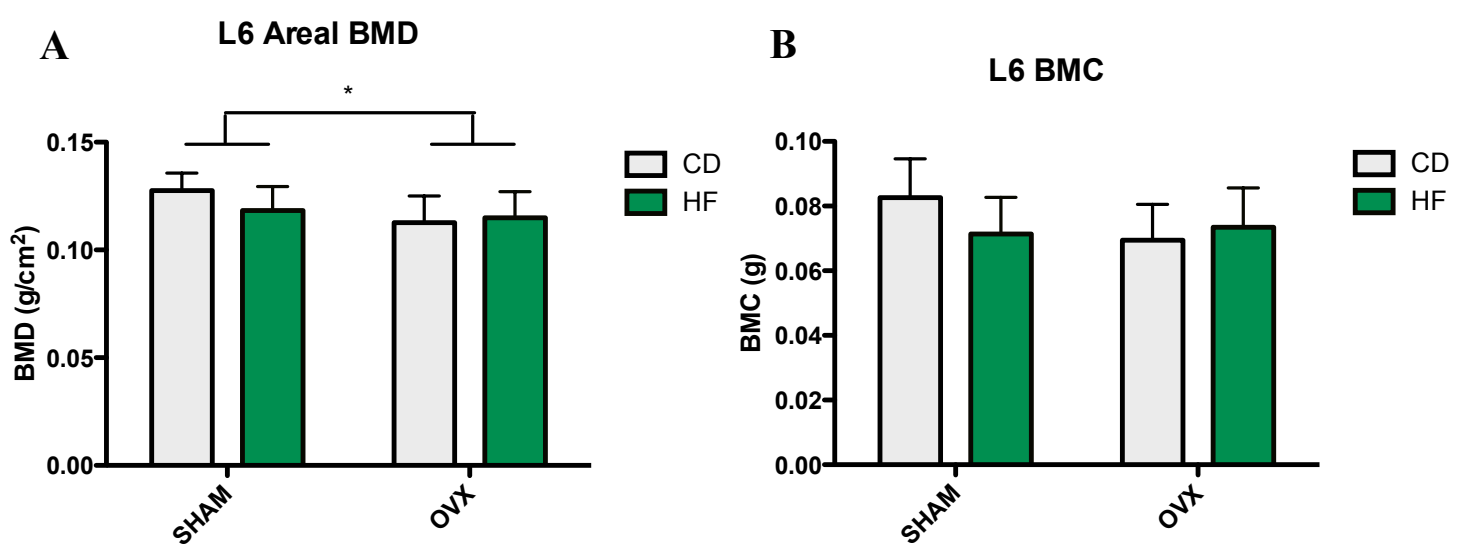

Figure 4-4. Results from DXA analysis of the L6 vertebrae from the surgical groups. (A) L6 aBMD (B) L6 BMC.Two-way ANOVA, mean \pm SD, * SHAM significantly different from OVX surgical groups. (A) diet alone $\mathrm{p}=0.3382$, surgery alone $\mathrm{p}=0.0155$, interaction $p=0.1191$. (B) diet alone $p=0.3571$, surgery alone $p=0.1693$, interaction $p=0.0598$.

\subsection{MICROCT ANALYSIS}

\subsubsection{FEMUR}

Left femurs were assessed by microCT analysis to investigate cortical vBMD and microarchitecture. Sample microCT cross-sectional images of the femur mid-diaphysis are shown in Figure 4-5. The vBMD and several microarchitecture parameters were unchanged between the baseline groups (Table 4-1). The cortical microarchitecture and geometry of the surgical groups was unaffected by the diet, surgery or an interaction of the two. No detectable differences were seen in the cross-sectional bone area, cortical thickness, medial-lateral diameter or anterior-posterior diameter (Table 4-2). For the vBMD, a two-way ANOVA showed there was not a detectable interaction between the effects of the diet and surgery, or diet alone; however it showed that OVX groups had a higher vBMD (Figure 4-6). 

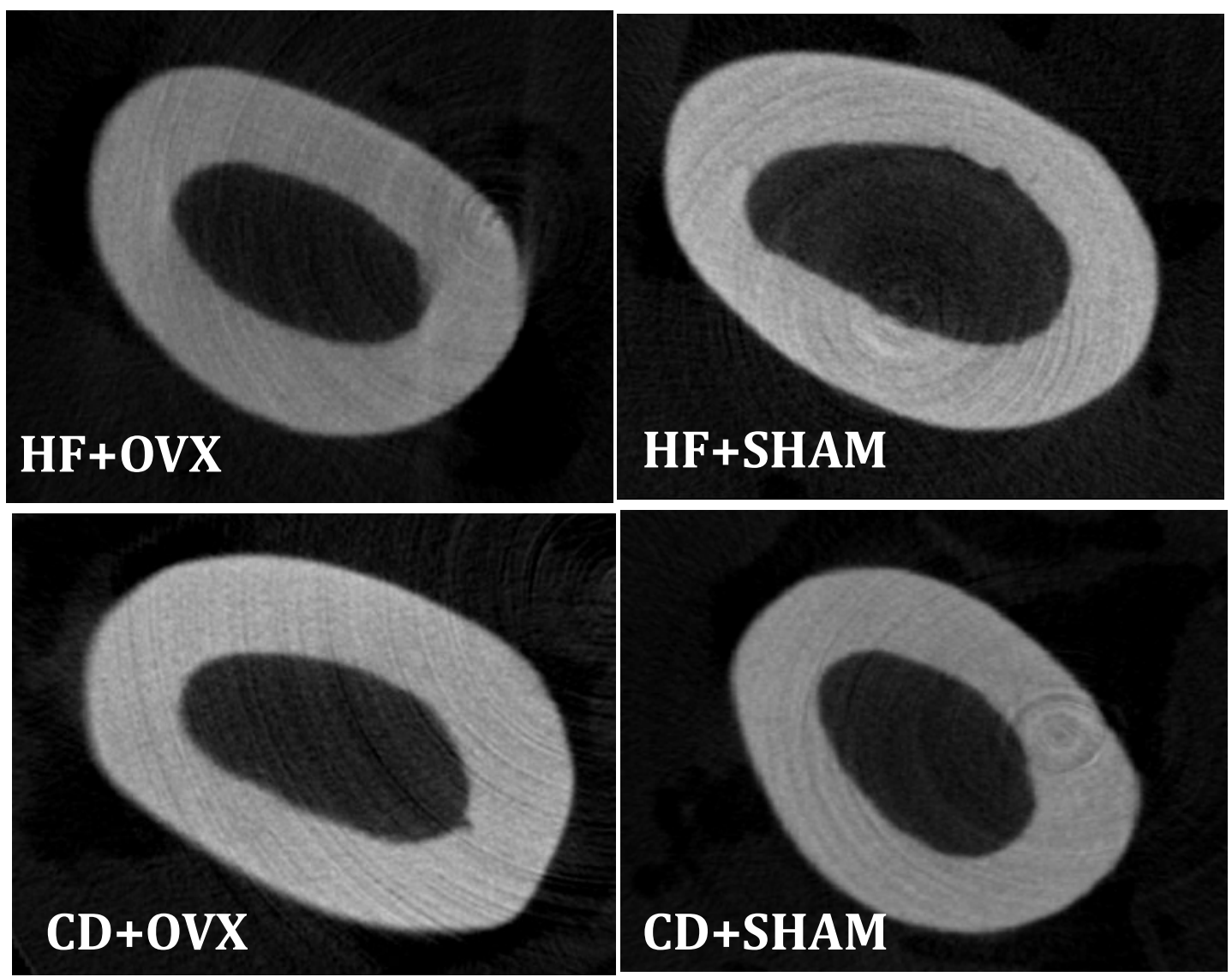

Figure 4-5. Representative single-slice microCT images of left femurs from the surgical groups at the mid-diaphysis.

Table 4-1. MicroCT results for femurs from baseline groups scanned at mid-diaphysis.

\begin{tabular}{lll}
\hline & HFB & CDB \\
\hline vBMD $\left(\mathbf{g} / \mathbf{c m}^{\mathbf{3}}\right)$ & $0.87 \pm 0.1$ & $0.88 \pm 0.07$ \\
$\begin{array}{l}\text { Cross Sectional Bone Area } \\
\left(\mathbf{m m}^{\mathbf{2}}\right)\end{array}$ & $6.83 \pm 0.4$ & $6.66 \pm 0.32$ \\
Cortical Thickness $(\mathbf{m m})$ & $0.77 \pm 0.04$ & $0.77 \pm 0.04$ \\
M-L Diameter $(\mathbf{m m})$ & $4.25 \pm 0.11$ & $4.29 \pm 0.23$ \\
A-P Diameter $(\mathbf{m m})$ & $3.21 \pm 0.14$ & $3.13 \pm 0.15$ \\
\hline Values reported as mean $\pm S D$. & & \\
\hline
\end{tabular}


Table 4-2. MicroCT results for femurs from surgical groups scanned at mid-diaphysis.

\begin{tabular}{lllll}
\hline & HF+OVX & HF+SHAM & CD+OVX & CD+SHAM \\
\hline $\begin{array}{l}\text { vBMD (g/ } \\
\mathbf{c m}^{\mathbf{3}} \text { ) }\end{array}$ & $1.00 \pm 0.02^{*}$ & $0.99 \pm 0.04$ & $1.01 \pm 0.07^{*}$ & $0.94 \pm 0.06$ \\
$\begin{array}{l}\text { Cross } \\
\text { Sectional Bone } \\
\text { Area }\left(\mathbf{m m}^{2}\right)\end{array}$ & $7.52 \pm 0.59$ & $7.16 \pm 0.47$ & $7.35 \pm 0.50$ & $7.29 \pm 0.48$ \\
$\begin{array}{l}\text { Cortical } \\
\text { Thickness }\end{array}$ & $0.80 \pm 0.05$ & $0.80 \pm 0.04$ & $0.79 \pm 0.04$ & $0.83 \pm 0.03$ \\
$\begin{array}{l}\text { (mm) } \\
\text { M-L Diameter } \\
(\mathbf{m m})\end{array}$ & $4.49 \pm 0.2$ & $4.38 \pm 0.23$ & $4.55 \pm 0.25$ & $4.48 \pm 0.21$ \\
$\begin{array}{l}\text { A-P Diameter } \\
(\mathbf{m m})\end{array}$ & $3.23 \pm 0.15$ & $3.23 \pm 0.14$ & $3.23 \pm 0.13$ & $3.18 \pm 0.17$ \\
\hline
\end{tabular}

Values reported as mean $\pm S D . *$ Significant $(p<0.05)$ compared to SHAM controls.

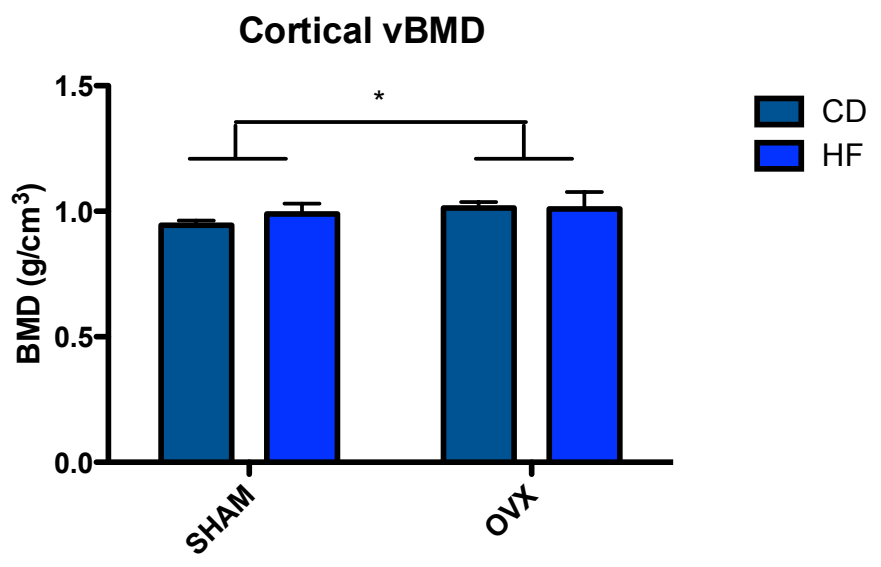

Figure 4-6. vBMD from microCT analysis of femurs from the surgical groups. Two-way ANOVA, mean $\pm \mathrm{SD}, \mathrm{n}=9$. * SHAM significantly different from OVX surgical groups. Diet alone $\mathrm{p}=0.2968$, surgery alone $\mathrm{p}=0.0310$, interaction $\mathrm{p}=0.2222$

\subsubsection{L6 VERTEBRAE}

L6 vertebrae were assessed by microCT analysis to investigate trabecular vBMD and microarchitecture. Sample microCT cross-sectional images of the vertebrae are shown in Figure 4-7. The vBMD and several microarchitecture parameters were unchanged between the baseline groups (Table 4-3). However, in the surgical groups 
several trabecular microarchitecture measurements were changed. A two-way ANOVA showed there was not a detectable interaction between the effects of diet and surgery or diet alone, however groups that underwent OVX surgery had a decreased percentage of trabecular bone volume, trabecular number and trabecular vBMD (Figure 4-8). Trabecular thickness, separation, cross-sectional bone area and tissue vBMD were unchanged between the surgical groups (Table 4-4). Tissue vBMD incorporates the entire trabecular tissue including both marrow space and cortical bone in the measurement, while trabecular vBMD measures the vBMD of trabeculae only.
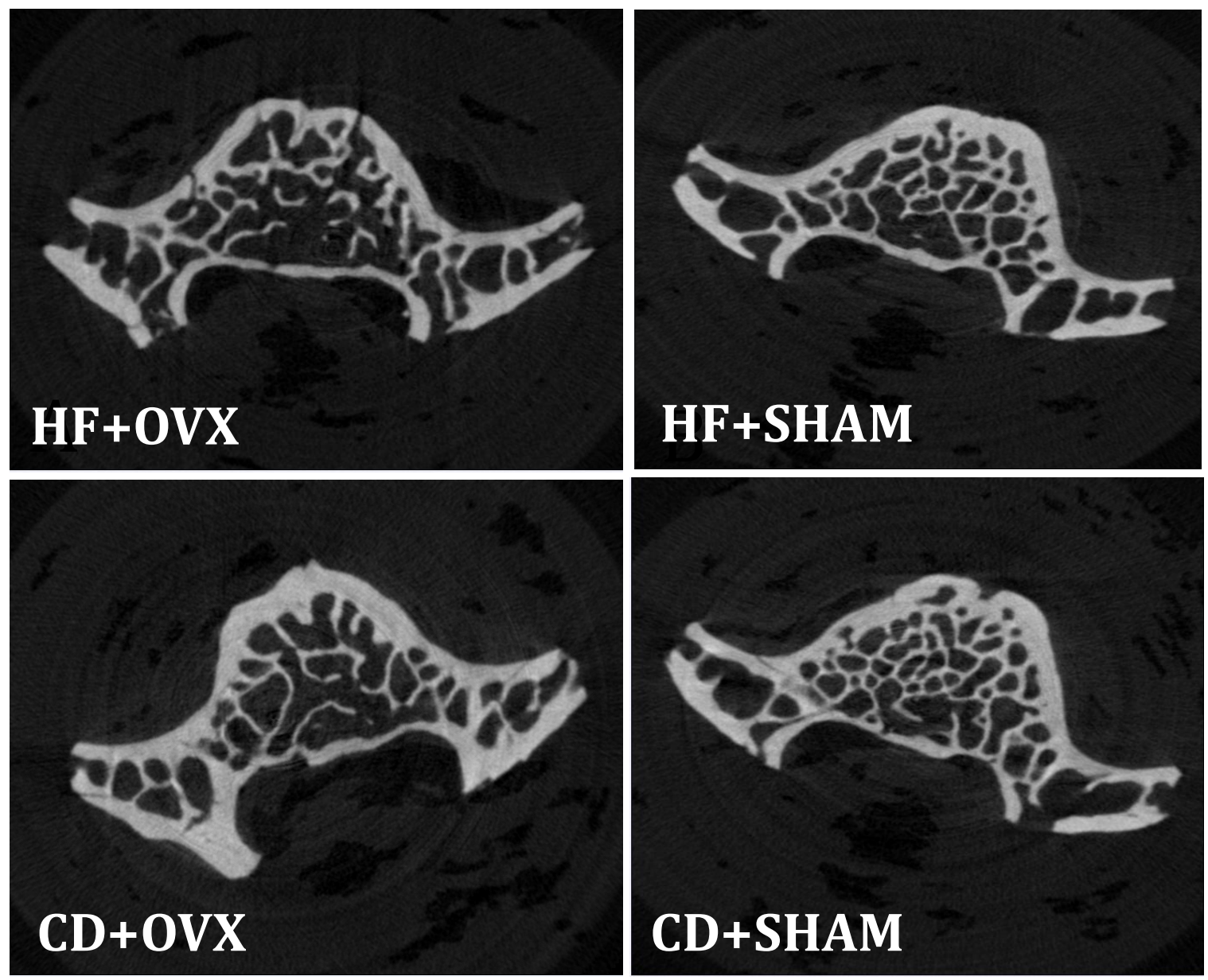

Figure 4-7. Representative mid-vertebral cross-sectional microCT images of the L6 vertebrae of surgical groups. 
Table 4-3. MicroCT results of secondary spongiosa in L6 vertebrae from baseline groups.

\begin{tabular}{|c|c|c|}
\hline & HFB & CDB \\
\hline $\begin{array}{l}\text { Trabecular Bone Volume } \\
(\%)\end{array}$ & $12.86 \pm 1.98$ & $12.75 \pm 1.56$ \\
\hline $\begin{array}{l}\text { Trabecular Thickness } \\
(\mu \mathrm{m})\end{array}$ & $0.10 \pm 0.004$ & $0.10 \pm 0.004$ \\
\hline $\begin{array}{l}\text { Trabecular Separation } \\
(\mu \mathrm{m})\end{array}$ & $0.89 \pm 0.09$ & $0.85 \pm 0.15$ \\
\hline $\begin{array}{l}\text { Trabecular Number } \\
(1 / \mathbf{m m})\end{array}$ & $1.27 \pm 0.16$ & $1.29 \pm 0.15$ \\
\hline $\begin{array}{l}\text { Mean Crossectional } \\
\text { Bone Area }\left(\mathrm{mm}^{2}\right)\end{array}$ & $5.67 \pm 0.67$ & $5.49 \pm 0.86$ \\
\hline $\begin{array}{l}\text { vBMD -tissue } \\
\left(\mathrm{g} / \mathrm{cm}^{3}\right)\end{array}$ & $0.74 \pm 0.03$ & $0.73 \pm 0.02$ \\
\hline $\begin{array}{l}\text { vBMD-trabeculae } \\
\left(\mathrm{g} / \mathrm{cm}^{3}\right)\end{array}$ & $0.31 \pm 0.08$ & $0.30 \pm 0.08$ \\
\hline
\end{tabular}

Table 4-4. MicroCT results of secondary spongiosa of L6 vertebrae from surgical groups.

\begin{tabular}{|c|c|c|c|c|}
\hline & HF+OVX & HF+SHAM & CD+OVX & CD+SHAM \\
\hline $\begin{array}{l}\text { Trabecular Bone } \\
\text { Volume }(\%)\end{array}$ & $10.53 \pm 1.75^{*}$ & $12.93 \pm 1.48$ & $10.95 \pm 0.96^{*}$ & $14.29 \pm 2.2$ \\
\hline $\begin{array}{l}\text { Trabecular Thickness } \\
(\mu \mathrm{m})\end{array}$ & $0.11 \pm 0.003$ & $0.10 \pm 0.004$ & $0.11 \pm 0.004$ & $0.11 \pm 0.00<$ \\
\hline $\begin{array}{l}\text { Trabecular Separation } \\
(\mu \mathrm{m})\end{array}$ & $0.92 \pm 0.09$ & $0.85 \pm 0.11$ & $0.89 \pm 0.06$ & $0.86 \pm 0.14$ \\
\hline $\begin{array}{l}\text { Trabecular Number } \\
(1 / \mathbf{m m})\end{array}$ & $1.0 \pm 0.15^{*}$ & $1.25 \pm 0.12$ & $1.0 \pm 0.08^{*}$ & $1.33 \pm 0.2$ \\
\hline $\begin{array}{l}\text { Mean Crossectional } \\
\text { Bone Area }\left(\mathrm{mm}^{2}\right)\end{array}$ & $5.62 \pm 1.20$ & $5.79 \pm 0.93$ & $4.95 \pm 0.67$ & $5.82 \pm 0.45$ \\
\hline $\begin{array}{l}\text { vBMD -tissue } \\
\left(\mathrm{g} / \mathrm{cm}^{3}\right)\end{array}$ & $0.80 \pm 0.04$ & $0.80 \pm 0.04$ & $0.79 \pm 0.03$ & $0.80 \pm 0.06$ \\
\hline $\begin{array}{l}\text { vBMD-trabeculae } \\
\left(\mathrm{g} / \mathrm{cm}^{3}\right)\end{array}$ & $0.32 \pm 0.1 *$ & $0.36 \pm 0.08$ & $0.32 \pm 0.07^{*}$ & $0.42 \pm 0.09$ \\
\hline
\end{tabular}

Values reported as mean $\pm S D$. * Significant $(p<0.05)$ compared to SHAM controls. 
A
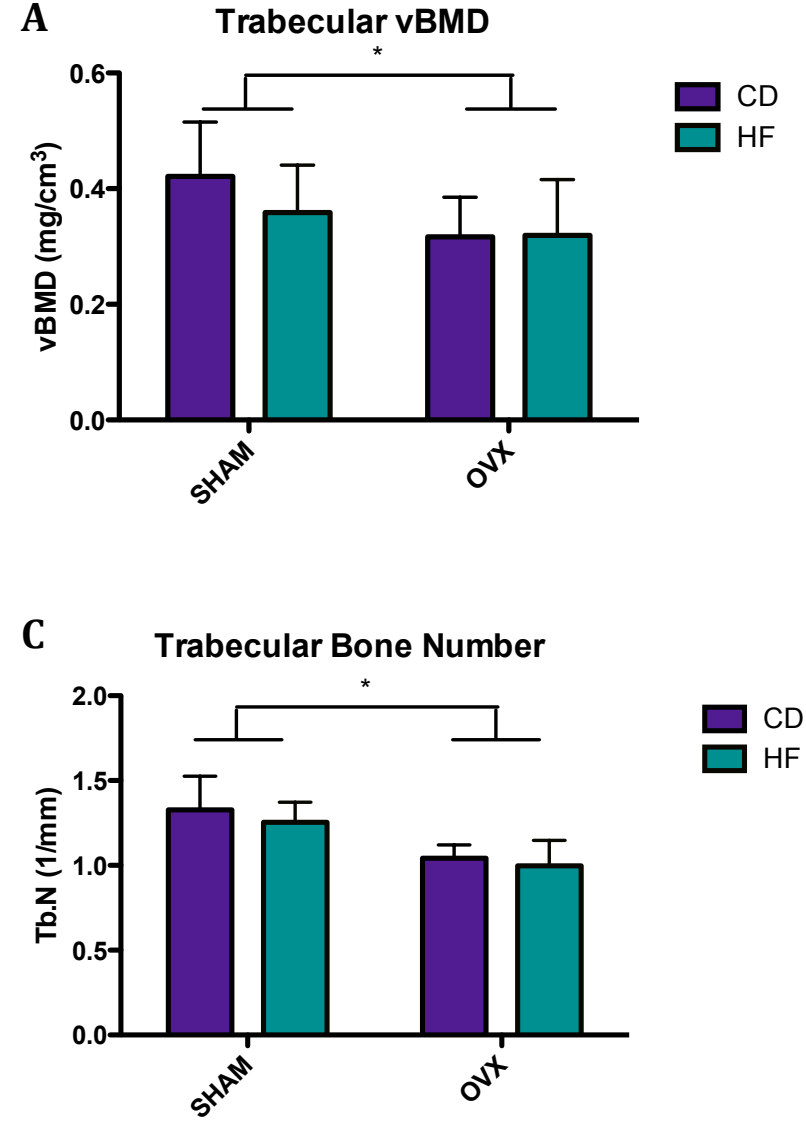

B Trabecular Bone Volume

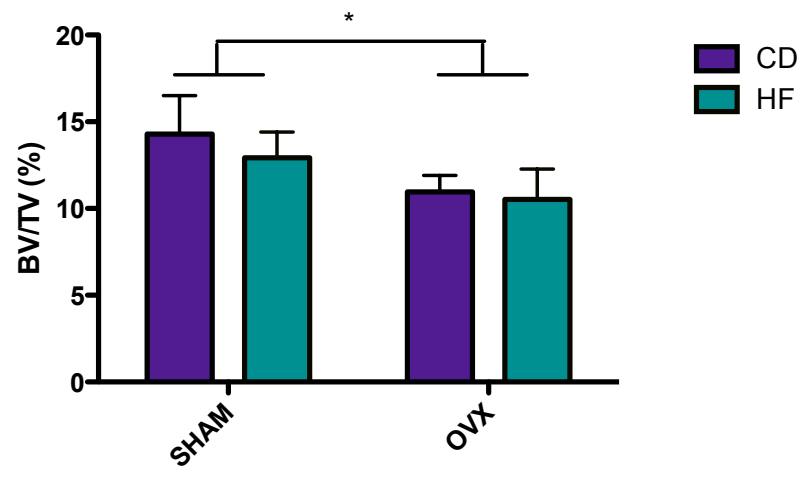

Figure 4-8. Results from microCT analysis of the L6 vertebrae from the surgical groups. (A) trabecular vBMD (B) trabecular bone volume (C) Trabecular bone number. Two-way ANOVA, mean $\pm \mathrm{SD}, \mathrm{n}=9$. * SHAM significantly different from OVX surgical groups. (A) diet alone $\mathrm{p}=0.311$, surgery alone $\mathrm{p}=0.019$, interaction $\mathrm{p}=0.271$ (B) diet alone $\mathrm{p}=0.128$, surgery alone $\mathrm{p}=0.000$, interaction $\mathrm{p}=0.418(\mathrm{C})$ diet alone $\mathrm{p}=0.234$, surgery alone $\mathrm{p}=0.000$, interaction $\mathrm{p}=0.766$.

\subsection{UNDECALCIFIED HISTOMORPHOMETRY}

Undecalcified histomorphometry is used to analyze bone formation parameters through the use of tibias embedded within Spurr resin. Specific analyses used are static histomorphometry performed with Goldner's Trichrome stain and dynamic histomorphometry performed with calcein green labeling. 


\subsubsection{GOLDNER'S TRICHROME STAIN}

Static histomorphometry was performed to measure the osteoid seam, which provides information about tissue-level formation. All osteoid measurements were unchanged between the baseline groups (Table 4-5). Several osteoid parameters measurements were changed within the surgical groups. A two-way ANOVA showed there was not a detectable interaction between the effects of diet and surgery or diet alone, however groups that underwent OVX surgery had increased OS, OS/BS and OV/BV. Osteoid width was increased due to the HFD but was unchanged due to the interaction of diet and surgery or surgery alone (Table 4-6 \& Figure 4-9).

Table 4-5. Results of histomorphometry on Goldner's Trichrome stained sections from baseline groups.

\begin{tabular}{lll}
\hline & HFB & CDB \\
\hline $\begin{array}{l}\text { Osteoid Surface } \\
\text { (mm) }\end{array}$ & $0.73 \pm 0.48$ & $1.04 \pm 0.69$ \\
$\begin{array}{l}\text { Osteoid Volume } \\
\left.\text { (mm }^{2}\right)\end{array}$ & $0.006 \pm 0.004$ & $0.008 \pm 0.006$ \\
$\begin{array}{l}\text { Osteoid } \\
\begin{array}{l}\text { Surface/Bone } \\
\text { Surface (\%) }\end{array}\end{array}$ & $0.010 \pm 0.008$ & $0.009 \pm 0.007$ \\
$\begin{array}{l}\text { Osteoid Volume/ } \\
\text { Bone Volume (\%) }\end{array}$ & $0.002 \pm 0.001$ & $0.002 \pm 0.002$ \\
$\begin{array}{l}\text { Osteoid Width } \\
(\mu \mathrm{m})\end{array}$ & $6.72 \pm 2.0$ & $7.34 \pm 1.68$ \\
\hline
\end{tabular}

Values reported as mean $\pm S D$. 
Table 4-6. Results of histomorphometry on Goldner's Trichrome stained sections from surgical groups.

\begin{tabular}{lllll}
\hline & HF+OVX & HF+SHAM & CD+OVX & CD+SHAM \\
\hline $\begin{array}{l}\text { Osteoid Surface } \\
(\mathbf{m m})\end{array}$ & $1.68 \pm 2.28^{*}$ & $0.78 \pm 0.65$ & $1.57 \pm 1.02^{*}$ & $0.38 \pm 0.34$ \\
$\begin{array}{l}\text { Osteoid Volume } \\
\left.\mathbf{( m m}^{2}\right)\end{array}$ & $0.02 \pm 0.03$ & $0.007 \pm 0.005$ & $0.009 \pm 0.01$ & $0.003 \pm 0.005$ \\
$\begin{array}{l}\text { Osteoid } \\
\begin{array}{l}\text { Surface/Bone } \\
\text { Surface (\%) }\end{array}\end{array}$ & $0.05 \pm 0.05^{*}$ & $0.01 \pm 0.01$ & $0.04 \pm 0.03^{*}$ & $0.005 \pm 0.004$ \\
$\begin{array}{l}\text { Osteoid Volume/ } \\
\text { Bone Volume (\%) }\end{array}$ & $0.008 \pm 0.008^{*}$ & $0.002 \pm 0.001$ & $0.006 \pm 0.003^{*}$ & $0.0008 \pm 0.0008$ \\
$\begin{array}{l}\text { Osteoid Width } \\
(\boldsymbol{\mu m})\end{array}$ & $7.55 \pm 3.01^{* *}$ & $10.15 \pm 4.57^{* *}$ & $5.94 \pm 1.79$ & $5.29 \pm 5.29$ \\
\hline
\end{tabular}

Values reported as mean $\pm S D$. * Significant $(p<0.05)$ compared to SHAM controls.

**Significant $(p<0.05)$ compared to $C D$.
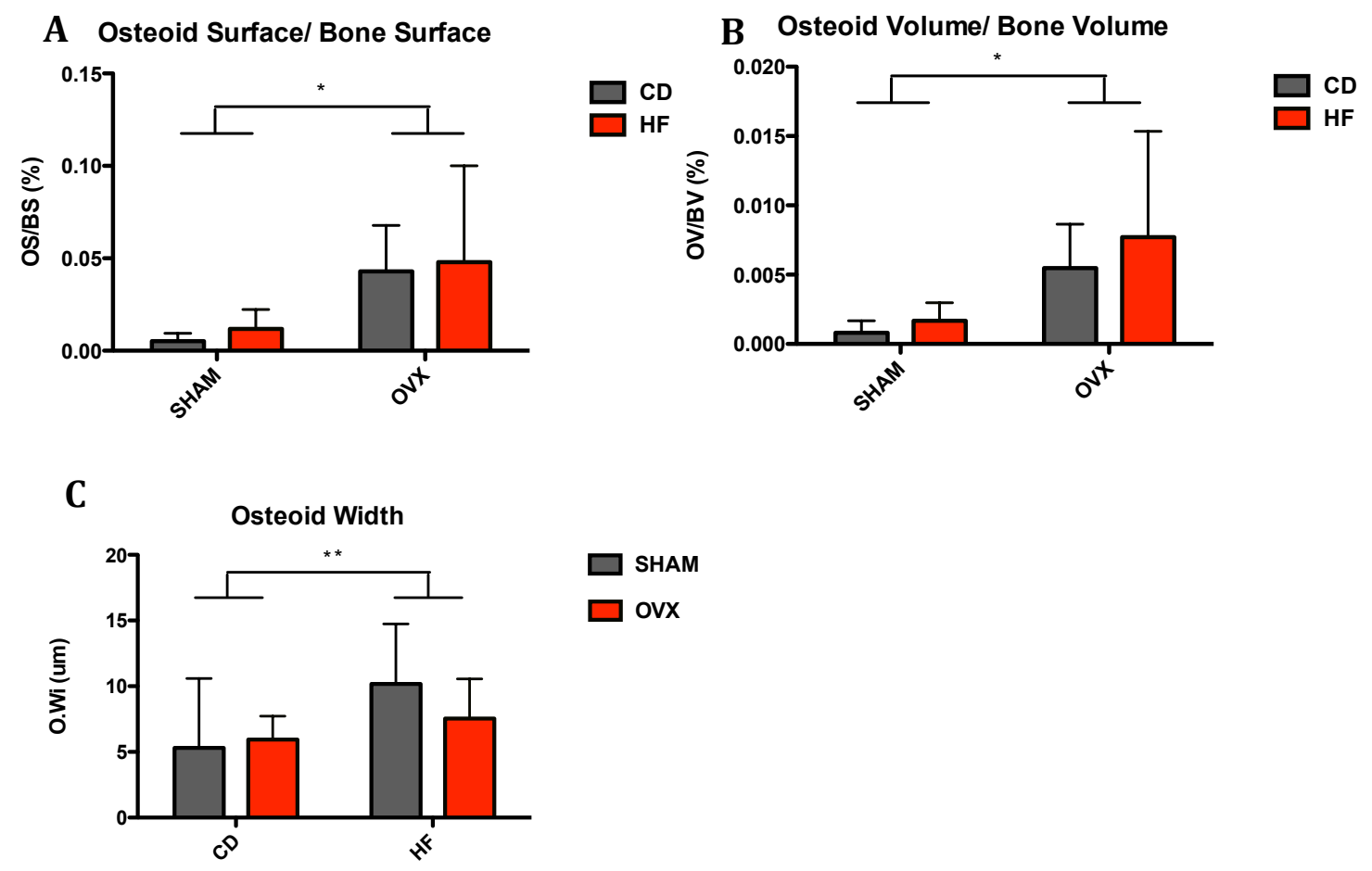

Fig 4-9. Results of histomorphometery for trichrome stained sections. (A) Osteoid surface per bone surface (B) osteoid volume per bone volume (C) osteoid width. Two-way ANOVA, mean $\pm \mathrm{SD}, \mathrm{n}=9$. * SHAM significantly different from OVX surgical groups, $* *$ CD significantly different from HFD groups (A) diet alone $\mathrm{p}=0.5475$ surgery alone $\mathrm{p}$ $=0.0005$, interaction $p=0.93(B)$ diet alone $p=0.2653$, surgery alone $p=0.0004$, interaction $\mathrm{p}=0.6209(\mathrm{C})$ diet alone $\mathrm{p}=0.0182$, surgery alone $\mathrm{p}=0.4588$ interaction $\mathrm{p}=0.2198$. 


\subsubsection{CALCEIN GREEN LABELING}

Dynamic histomorphometry was performed to quantify mineralization rates. All dynamic measurements were unchanged between the baseline groups (Table 4-7). Twoway ANOVA analysis showed that OVX alone caused an increase in dLS, Ir.L.Width, MS/BS, MAR BFR/BS and BFR/BV (Table 4-8 \& Figures 4-10 \& 4-11). Neither the diet nor the interaction of the diet and surgery caused changes in these factors. The SHAM surgery alone caused an increase in the sLS. (Table 4-8 \& Figure 4-10).

Table 4-7. Results of dynamic histomorphometry in baseline groups.

\begin{tabular}{|c|c|c|}
\hline & HFB & CDB \\
\hline $\begin{array}{l}\text { Single Label Surface } \\
\text { (mm) }\end{array}$ & $18.12 \pm 6.79$ & $21.23 \pm 7.4$ \\
\hline $\begin{array}{l}\text { Double Label Surface } \\
\text { (mm) }\end{array}$ & $8.06 \pm 3.54$ & $8.47 \pm 4.39$ \\
\hline $\begin{array}{l}\text { Mineralizing Surface } \\
\text { (mm) }\end{array}$ & $17.12 \pm 5.1$ & $19.09 \pm 6.4$ \\
\hline Interlabel Width $(\mu \mathrm{m})$ & $26.89 \pm 4.31$ & $25.21 \pm 1.86$ \\
\hline $\begin{array}{l}\text { Mineralizing } \\
\text { Surface/Bone Surface } \\
(\%)\end{array}$ & $0.22 \pm 0.08$ & $0.26 \pm 0.12$ \\
\hline $\begin{array}{l}\text { Mineral Apposition } \\
\text { Rate }(\mu \mathrm{m} / \text { day })\end{array}$ & $3.84 \pm 0.62$ & $3.6 \pm 0.27$ \\
\hline $\begin{array}{l}\text { Bone Formation Rate/ } \\
\text { Bone Surface } \\
(\mu \mathrm{m} / \text { day } / \mathbf{m m})\end{array}$ & $0.85 \pm 0.39$ & $0.93 \pm 0.46$ \\
\hline $\begin{array}{l}\text { Bone Formation Rate/ } \\
\text { Bone Volume } \\
\left(\mu \mathrm{m} / \mathrm{day} / \mathbf{m m}^{2}\right)\end{array}$ & $17.5 \pm 8.75$ & $22.17 \pm 13.54$ \\
\hline
\end{tabular}


Table 4-8. Results of dynamic histomorphometry in surgical groups.

\begin{tabular}{|c|c|c|c|c|}
\hline & HF+OVX & HF+SHAM & $\mathrm{CD}+\mathrm{OVX}$ & CD+SHAM \\
\hline $\begin{array}{l}\text { Single Label } \\
\text { Surface }(\mathrm{mm})\end{array}$ & $6.84 \pm 3.6^{*}$ & $13.24 \pm 4.23$ & $7.43 \pm 3.16^{*}$ & $13.62 \pm 5.92$ \\
\hline $\begin{array}{l}\text { Double Label } \\
\text { Surface }(\mathrm{mm})\end{array}$ & $5.20 \pm 3.48 *$ & $3.8 \pm 1.77$ & $5.21 \pm 2.29 *$ & $1.83 \pm 1.14$ \\
\hline $\begin{array}{l}\text { Mineralizing } \\
\text { Surface (mm) }\end{array}$ & $8.62 \pm 3.78$ & $10.42 \pm 3.29$ & $8.92 \pm 2.95$ & $8.64 \pm 3.88$ \\
\hline $\begin{array}{l}\text { Interlabel Width } \\
(\mu \mathrm{m})\end{array}$ & $25.74 \pm 4.27 *$ & $23.01 \pm 1.73$ & $24.97 \pm 3.38^{*}$ & $21.82 \pm 2.92$ \\
\hline $\begin{array}{l}\text { Mineralizing } \\
\text { Surface/Bone } \\
\text { Surface }(\%)\end{array}$ & $0.29 \pm 0.17^{*}$ & $0.18 \pm 0.14$ & $0.26 \pm 0.14^{*}$ & $0.12 \pm 0.06$ \\
\hline $\begin{array}{l}\text { Mineral } \\
\text { Apposition Rate } \\
(\mu \mathrm{m} / \text { day })\end{array}$ & $3.68 \pm 0.61 *$ & $3.29 \pm 0.25$ & $3.57 \pm 0.48^{*}$ & $3.12 \pm 0.42$ \\
\hline $\begin{array}{l}\text { Bone Formation } \\
\text { Rate/ Bone } \\
\text { Surface } \\
(\mu \mathrm{m} / \mathrm{day} / \mathrm{mm})\end{array}$ & $1.07 \pm 0.65^{*}$ & $0.61 \pm 0.5$ & $0.94 \pm 0.56^{*}$ & $0.39 \pm 0.21$ \\
\hline $\begin{array}{l}\text { Bone Formation } \\
\text { Rate/ Bone } \\
\text { Volume } \\
\left(\mu \mathrm{m} / \mathrm{day} / \mathbf{m m}^{2}\right)\end{array}$ & $22.41 \pm 15.25^{*}$ & $9.36 \pm 6.5$ & $19.83 \pm 12.31 *$ & $9.65 \pm 6.82$ \\
\hline
\end{tabular}

Values reported as mean $\pm S D$. * Significant $(p<0.05)$ compared to SHAM controls. 
A
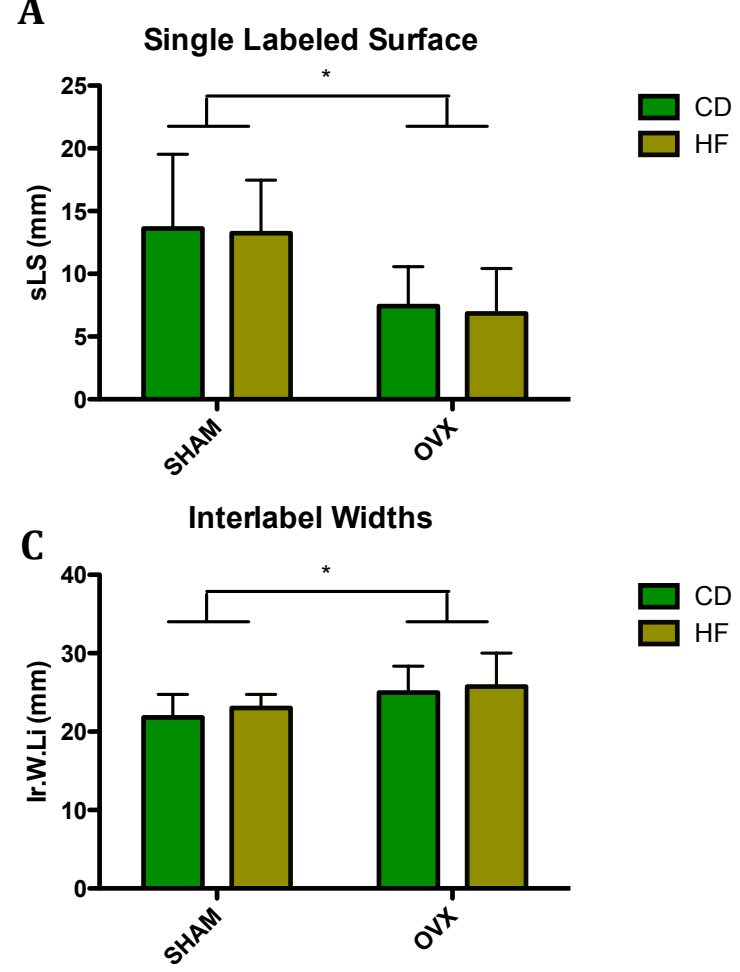

B Double Labeled Surface

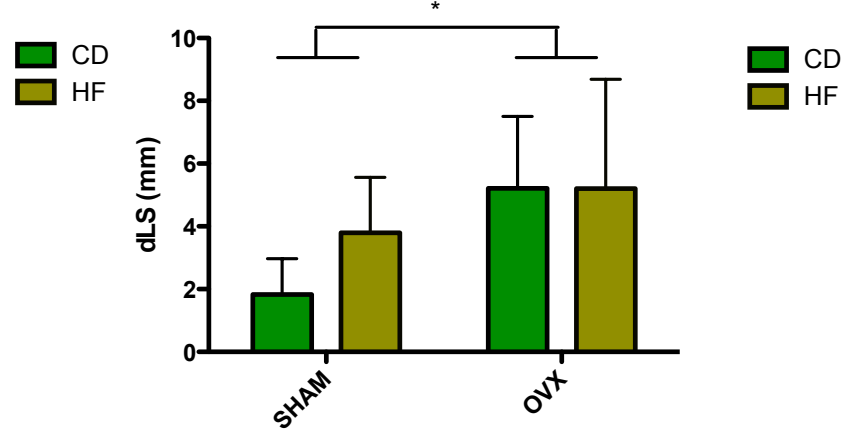

Fig 4-10. Results of dynamic histomorphometry. (A) single labeled surface (B) double labeled surface $(C)$ interlabel width. Two-way ANOVA, mean $\pm \mathrm{SD}, \mathrm{n}=9$. ${ }^{*} \mathrm{SHAM}$ significantly different from OVX surgical groups (A) diet alone $\mathrm{p}=0.7401$, surgery alone $p=0.0001$, interaction $p=0.9431$ (B) diet alone $p=0.2163$, surgery alone $p=0.0043$, interaction $\mathrm{p}=0.2136(\mathrm{C})$ diet alone $\mathrm{p}=0.3656$, surgery alone $\mathrm{p}=0.0098$, interaction $\mathrm{p}=0.8453$. 


\section{A}

Mineralizing Surface/Bone Surface

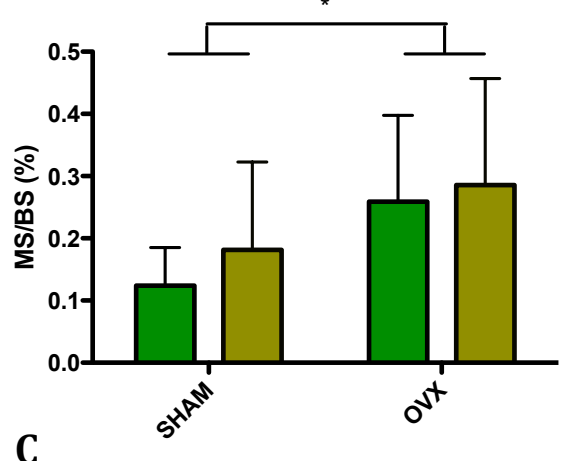

Bone formation rate/bone surface

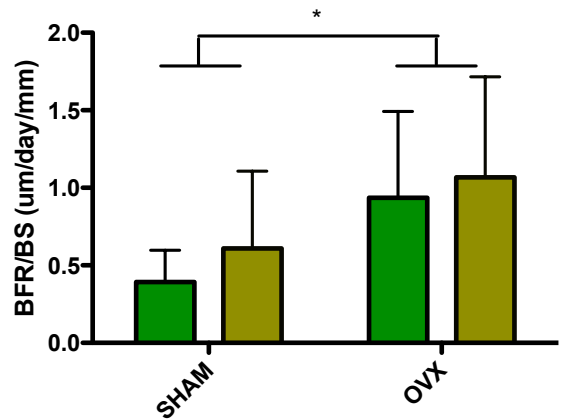

B

Mineral Apposition Rate

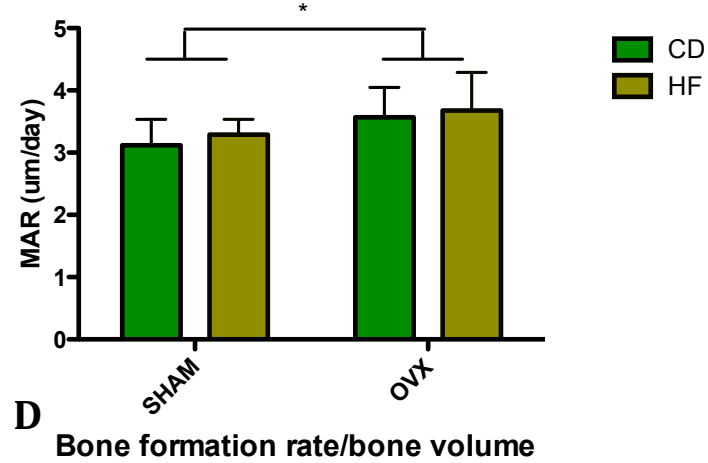

$\square \mathrm{CD}$

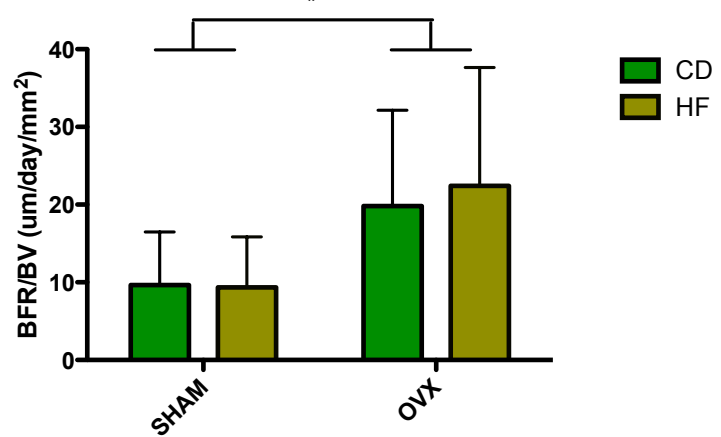

Fig 4-11. Results of dynamic histomorphometry. (A) mineralizing surface per bone surface (B) mineral apposition rate (C) bone formation rate per bone surface (D) bone formation rate per bone volume. Two-way ANOVA, Mean \pm SD, n=9. *SHAM significantly different from OVX surgical groups.(A) diet alone $\mathrm{p}=0.3557$, surgery alone $\mathrm{p}=0.0117$, interaction $\mathrm{p}=0.7341(\mathrm{~B})$ diet alone $\mathrm{p}=0.3656$, surgery alone $\mathrm{p}=0.0098$, diet $\&$ surgery $p=0.8453(\mathrm{C})$ diet alone $\mathrm{p}=0.3092$, surgery alone $\mathrm{p}=0.0055$, interaction $\mathrm{p}=0.8(\mathrm{D})$ diet alone $\mathrm{p}=0.7539$, surgery alone $\mathrm{p}=0.0031$, interaction $\mathrm{p}=0.6948$.

\subsection{DECALCIFIED HISTOMORPHOMETRY}

Decalcified histomorphometry was used to analyze bone resorption parameters. All TRAP measurements were unchanged in both the baseline (Table 4-9) and surgical groups (Table 4-10). However, several trends were noted after performing a two-way ANOVA analysis in the surgical groups. The interaction of the HFD and OVX surgery trended towards having the greatest N.Oc/BS $(p=0.069)$ and Oc.S/BS $(p=0.069)$ while the diet or surgery alone did not seem to have a detectable effect. 
Table 4-9. Results of histomorphometry analysis of TRAP stained sections for baseline groups.

\begin{tabular}{lll}
\hline & HFB & CDB \\
\hline $\begin{array}{l}\text { Number of osteoclasts } \\
\text { (\#) }\end{array}$ & $100.8 \pm 23.2$ & $95.8 \pm 32.9$ \\
$\begin{array}{l}\text { Osteoclast Surface } \\
\text { (mm) }\end{array}$ & $10.59 \pm 2.68$ & $10.89 \pm 5.77$ \\
$\begin{array}{l}\text { Number of } \\
\text { osteoclasts/bone } \\
\text { surface (1/mm) }\end{array}$ & $0.78 \pm 0.27$ & $0.65 \pm 0.2$ \\
$\begin{array}{l}\text { Osteoclast } \\
\text { surface/bone surface } \\
(\%)\end{array}$ & $0.09 \pm 0.04$ & $0.07 \pm 0.04$ \\
\hline
\end{tabular}

Values reported as mean $\pm S D$.

Table 4-10. Results of histomorphometry analysis of TRAP stained sections for surgical groups.

\begin{tabular}{lllll}
\hline & HF+OVX & HF+SHAM & CD+OVX & CD+SHAM \\
\hline $\begin{array}{l}\text { Number of } \\
\text { osteoclasts (\#) }\end{array}$ & $64.22 \pm 49.11$ & $80.89 \pm 38.1$ & $43.75 \pm 21.63$ & $120.1 \pm 39.27$ \\
$\begin{array}{l}\text { Osteoclast Surface } \\
\text { (mm) }\end{array}$ & $7.52 \pm 0.59$ & $7.16 \pm 0.47$ & $7.35 \pm 0.5$ & $7.29 \pm 0.48$ \\
$\begin{array}{l}\text { Number of } \\
\text { osteoclasts/bone } \\
\text { surface (1/mm) }\end{array}$ & $1.05 \pm 0.42^{*}$ & $0.77 \pm 0.35$ & $0.76 \pm 0.22$ & $0.87 \pm 0.19$ \\
$\begin{array}{l}\text { Osteoclast } \\
\text { surface/bone surface } \\
\text { (\%) }\end{array}$ & $0.07 \pm 0.05^{*}$ & $0.06 \pm 0.04$ & $0.05 \pm 0.14$ & $0.07 \pm 0.02$ \\
\hline
\end{tabular}

Values reported as mean $\pm S D$. ${ }^{*}$ Trend $(0.07>p>0.05)$ interaction of $H F+O V X$ having greatest amount.

\subsection{HEMATOXYLIN AND EOSIN STAINING}

Histological analysis of stained sections of paraffin-embedded rat liver was performed to determine whether the rats fed the HFD developed NAFLD. Specifically, hematoxylin and eosin staining was done to analyze steatosis, hepatic inflammation, and fibrosis of the liver. Upon staining, it was clear that there was no indication of any of the 
previously mentioned parameters in the baseline (Figure 4-12) and surgical groups

(Figure 4-13) therefore further analysis was not completed.
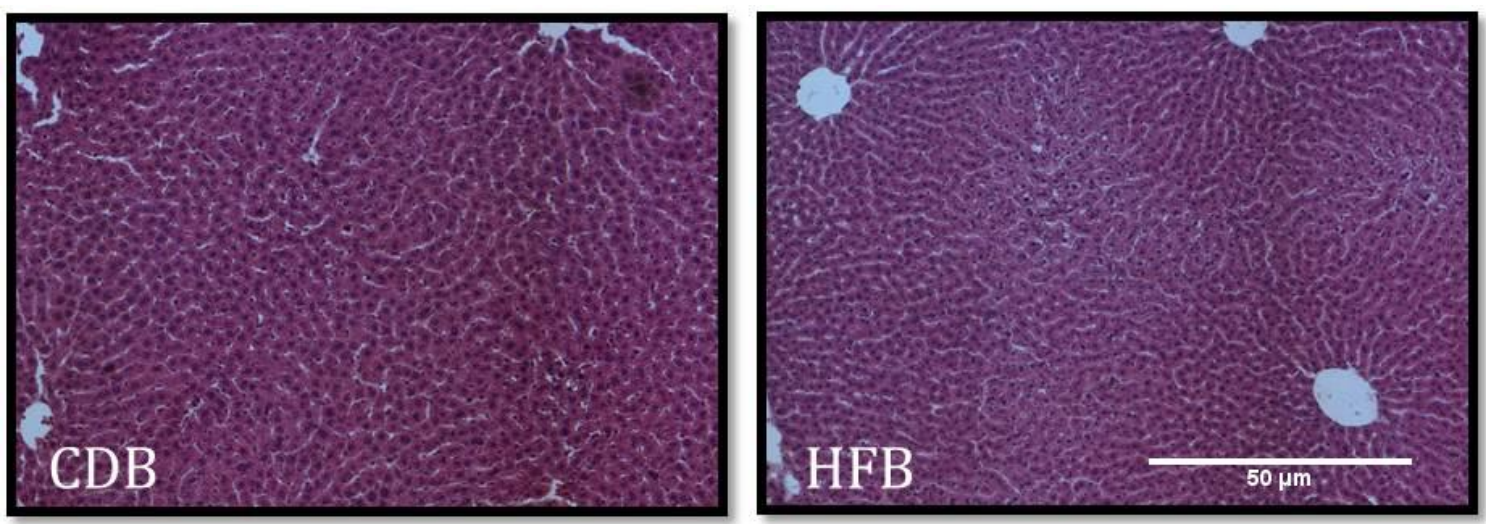

Figure 4-12. Representative images of liver sections from baseline groups stained with hematoxylin and eosin.
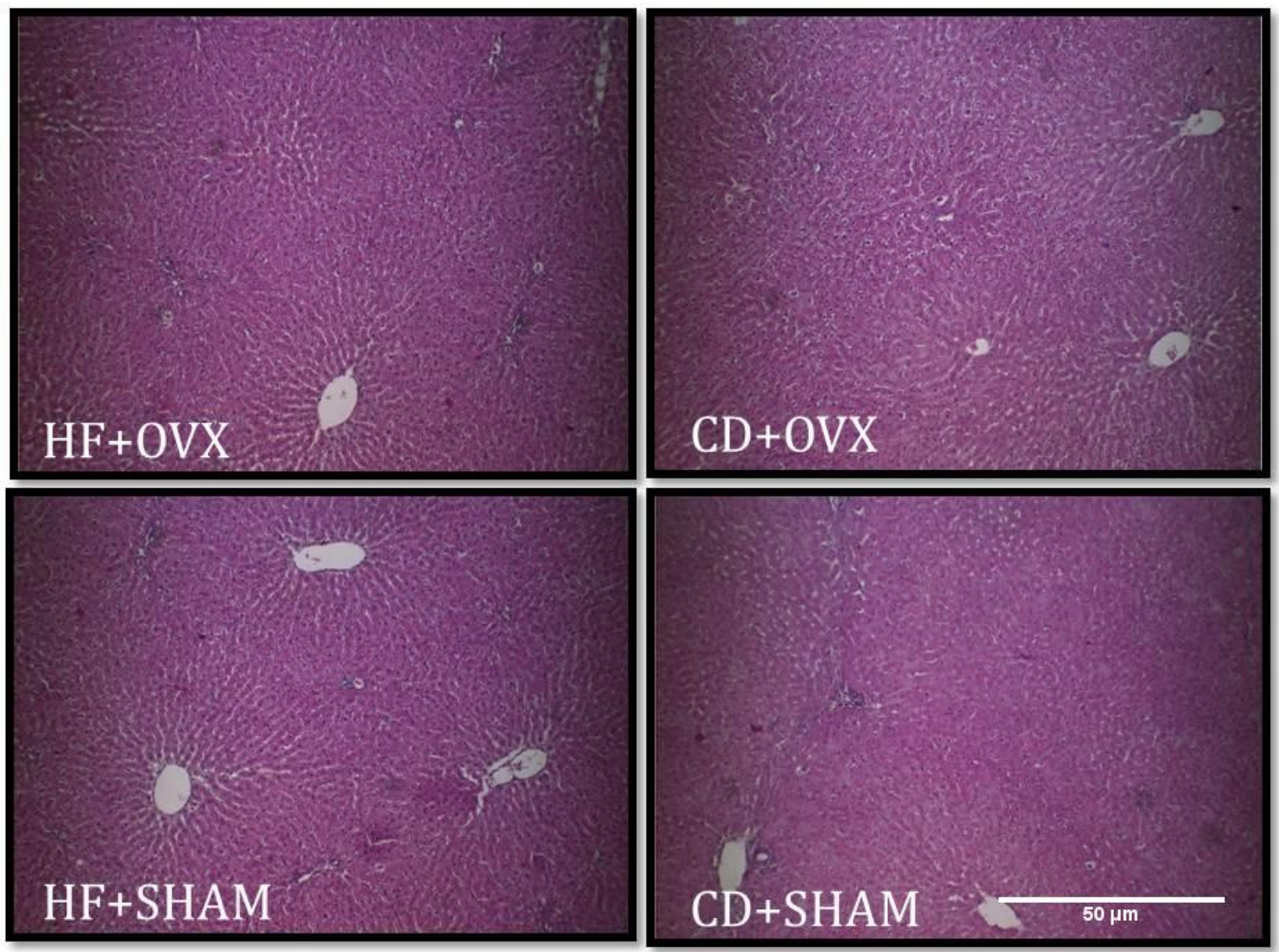

Figure 4-13. Representative images of liver sections from surgical groups stained with hematoxylin and eosin. 


\subsection{ELISA}

ELISAs were used to analyze soluble pentosidine, CML and RAGE in serum. ELISAs were performed on serum samples taken at four time-points throughout the study; 1) at start, before diet was implemented 2) after 3 months on diet 3) one month after surgeries 4 ) at the end of the study, 3 months after surgery. All pentosidine, CML and sRAGE levels taken at time-points 1 and 2 were taken before surgical intervention, and therefore were analyzed by a student's t-test, grouping all rats on the HFD together and all rats on CD together. Pentosidine, CML and sRAGE levels taken at time-points 3 and 4 were taken after surgical intervention, and were analyzed by two-way ANOVAs, comparing the four surgical groups separately.

\subsubsection{SOLUBLE PENTOSIDINE}

At time-points 1 and 2, soluble pentosidine was unchanged between the two diet conditions (Table 4-11). At time points 3 and 4, there was no change in pentosidine levels between the groups due to diet, surgery or an interaction between the two factors (Table 4-12).

To analyze changes between the time-points paired t-tests were used (Figure 414). All groups had a significant increase in pentosidine levels from time-point 1 to timepoint 2 ( $p<0.05$ for all groups), no changes were noted between time-point 2 and 3 and only the CD+OVX group saw a significant decrease $(p=0.005)$ between time-point 3 and 4. 
Table 4-11. Results of ELISA for soluble pentosidine at time-points $1 \& 2$.

\begin{tabular}{lll}
\hline & HF & CD \\
\hline $\begin{array}{l}\text { Pentosidine (beginning of } \\
\text { study, ng/mL) }\end{array}$ & $1.54 \pm 1.03$ & $1.42 \pm 0.49$ \\
$\begin{array}{l}\text { Pentosidine (after } 3 \text { months } \\
\text { of diet, ng/mL) }\end{array}$ & $2.67 \pm 0.67$ & $3.16 \pm 1.51$ \\
\hline $\begin{array}{l}\text { Values reported as mean } \pm S D . \\
\text { S }\end{array}$ & & \\
\hline
\end{tabular}

Table 4-12. Results of ELISA for soluble pentosidine for surgical groups at all timepoints.

\begin{tabular}{lllll}
\hline & HF+OVX & HF+SHAM & CD+OVX & CD+SHAM \\
\hline $\begin{array}{l}\text { Pentosidine (beginning } \\
\text { of study, ng/mL) }\end{array}$ & $1.75 \pm 0.84$ & $1.57 \pm 1.5$ & $1.12 \pm 0.39$ & $1.6 \pm 0.53$ \\
\hline $\begin{array}{l}\text { Pentosidine (after 3 } \\
\text { months of diet, ng/mL) }\end{array}$ & $2.71 \pm 0.76$ & $2.86 \pm 0.69$ & $2.49 \pm 0.55$ & $2.82 \pm 0.6$ \\
$\begin{array}{l}\text { Pentosidine (1 month } \\
\text { after surgery, ng/mL) }\end{array}$ & $3.26 \pm 2.11$ & $2.4 \pm 1.25$ & $2.65 \pm 0.5$ & $2.64 \pm 0.73$ \\
$\begin{array}{l}\text { Pentosidine (3 months } \\
\text { after surgery, ng/mL) }\end{array}$ & $2.31 \pm 1.26$ & $2.47 \pm 1.59$ & $2.07 \pm 0.59$ & $2.61 \pm 2.80$ \\
\hline \begin{tabular}{l} 
Values reported as mean $\pm S D$. \\
\hline
\end{tabular} & & & &
\end{tabular}

\section{Pentosidine}

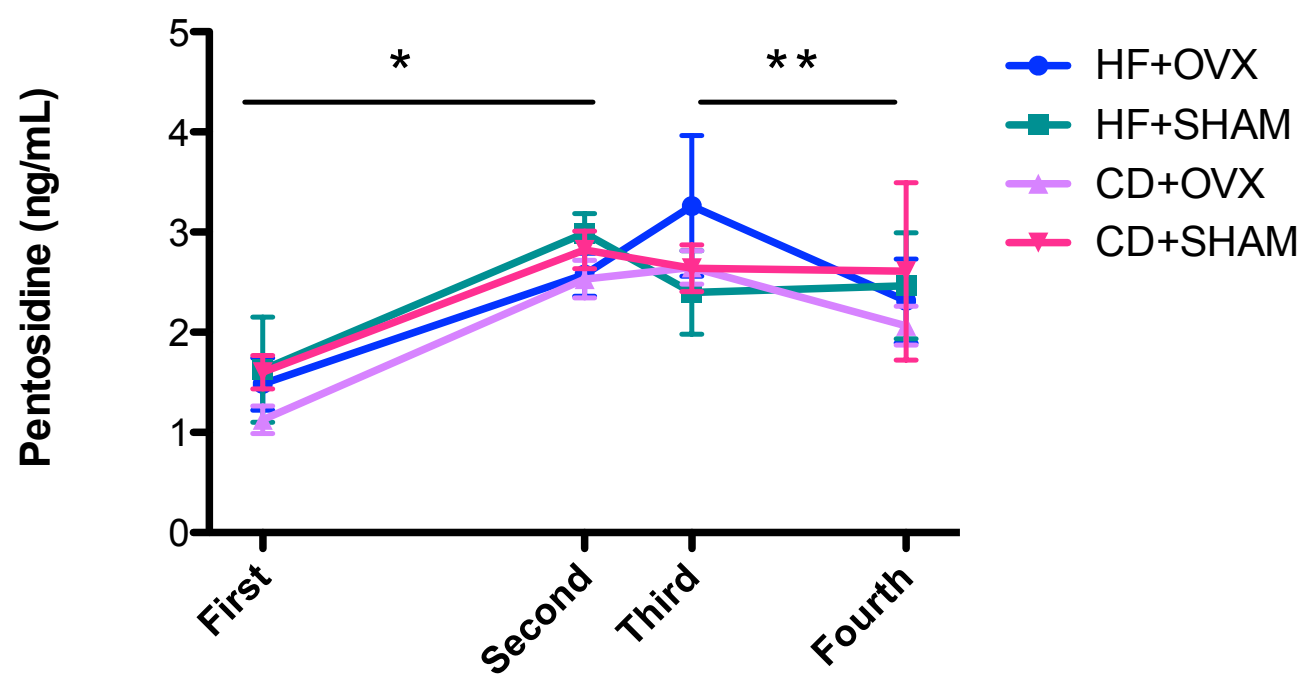

Figure 4-14. ELISA results for pentosidine at each time-point for surgical groups. Paired $\mathrm{T}$-Test, mean $\pm \mathrm{SD}$. ${ }^{*}$ Significant increase in pentosidine for all groups $(\mathrm{p}<0.05) ; * *$ significant decrease in pentosidine for CD+OVX $(p=0.005)$. 


\subsubsection{SOLUBLE CML}

At time-points 1 and 2, soluble CML was unchanged between the two diet conditions (Table 4-13). At time-point 4, there was no change in CML levels between the groups due to diet, surgery or a combination of the two factors. However, at time-point 3 the groups fed the CD expressed more soluble CML, while surgery and the interaction of diet and surgery did not have a detectable effect. (Table 4-14 \& Figure 4-15).

To analyze changes between time-points paired t-tests were used (Figure 4-16). All groups had a significant increase in CML levels from time-point 1 to time-point 2 ( $p<0.05$ for all groups), no changes were noted between time-point 2 and 3 , while the HF+SHAM $(p=0.001)$, CD+OVX $(p=0.008), C D+\operatorname{SHAM}(p=0.018)$ groups saw a significant decrease between time-points 3 and 4 (Figure 4-16).

Table 4-13. Results of ELISA for CML at time-points $1 \& 2$.

\begin{tabular}{lll}
\hline & HF & CD \\
\hline $\begin{array}{l}\text { CML (beginning of study, } \\
\text { ng/mL) }\end{array}$ & $6.69 \pm 2.08$ & $6.92 \pm 1.52$ \\
$\begin{array}{l}\text { CML (after } 3 \text { months of } \\
\text { diet, ng/mL) }\end{array}$ & $10.95 \pm 3.12$ & $11.24 \pm 3.06$ \\
\hline $\begin{array}{l}\text { Values reported as mean } \pm S D . \\
\end{array}$ &
\end{tabular}

Table 14. Results of ELISA for CML for surgical groups at all time-points.

\begin{tabular}{lllll}
\hline & HF+OVX & HF+SHAM & CD+OVX & CD+SHAM \\
\hline $\begin{array}{l}\text { CML (beginning of study, } \\
\text { ng/mL) }\end{array}$ & $6.98 \pm 2.64$ & $6.46 \pm 2.07$ & $7.58 \pm 1.59$ & $6.94 \pm 1.45$ \\
$\begin{array}{l}\text { CML (after 3 months of } \\
\text { diet, ng/mL) }\end{array}$ & $11.86 \pm 3.53$ & $10.83 \pm 3.05$ & $13.79 \pm 1.98$ & $11.53 \pm 2.63$ \\
$\begin{array}{l}\text { CML (1 month after } \\
\text { surgery, ng/mL) }\end{array}$ & $10.05 \pm 3.72$ & $9.9 \pm 1.23$ & $13.35 \pm 3.14^{*}$ & $10.97 \pm 1.97^{*}$ \\
$\begin{array}{l}\text { CML (3 months after } \\
\text { surgery, ng/mL) }\end{array}$ & $8.22 \pm 2.62$ & $7.41 \pm 1.27$ & $9.5 \pm 1.88$ & $8.64 \pm 2.94$ \\
\hline $\begin{array}{l}\text { Values reported as mean } \pm S D . \\
\text { * Significant }(p<0.05) \text { compared to HFD groups. }\end{array}$ &
\end{tabular}




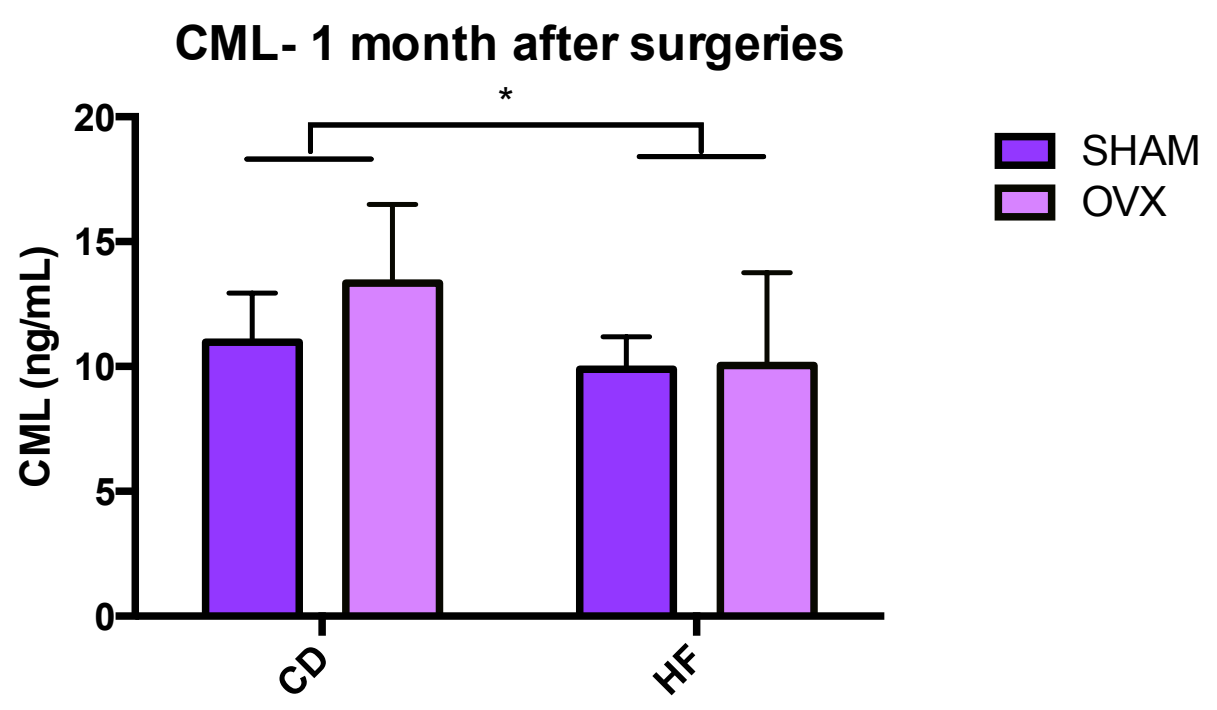

Fig 4-15. ELISA results for circulating CML measured one month after surgeries. Twoway ANOVA, Mean $\pm \mathrm{SD}, \mathrm{n}=9 .{ }^{*} \mathrm{CD}$ significantly different from HFD groups. Diet alone $\mathrm{p}=0.019$, surgery alone $\mathrm{p}=0.162$, interaction $\mathrm{p}=0.216$.

\section{CML}

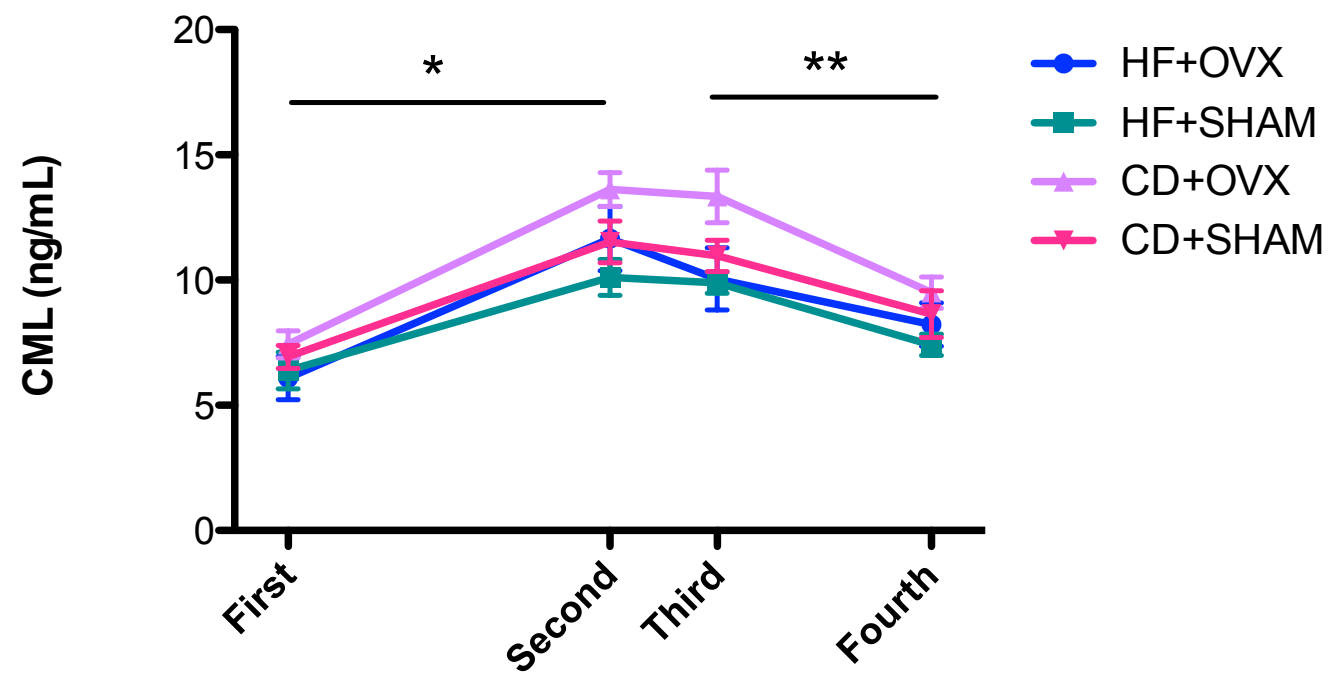

Figure 4-16. ELISA results for CML at each time-point for surgical groups. Paired TTest, mean \pm SD. * Significant increase in CML for all groups $(\mathrm{p}<0.05) ; * *$ significant decrease in CML for HF+SHAM ( $\mathrm{p}=0.001), \mathrm{CD}+\mathrm{OVX}(\mathrm{p}=0.008)$, and $\mathrm{CD}+\mathrm{SHAM}$ $(\mathrm{p}=0.018)$. 


\subsubsection{SOLUBLE RAGE (sRAGE)}

At time-points 1\&2, sRAGE was unchanged between the two diet conditions (Table 4-15). At time-point 4, there was no change in sRAGE levels between the groups due to diet, surgery or an interaction between the two factors. At time-point 3, the groups that underwent OVX surgeries significantly expressed more sRAGE, while diet and an interaction of diet and surgery had no effect (Table 4-16 \& Figure 4-17).

To analyze changes between the time-points, paired t-tests were used (Figure 418). All groups had a significant increase in sRAGE levels from time-point 1 to timepoint 2 ( $p<0.05$ for all groups); between time-points 2 and $3 \mathrm{HF}+\mathrm{OVX}(\mathrm{p}=0.043)$, HF+SHAM $(p=0.042)$ and CD+SHAM $(p=0.015)$ saw a significant decrease, and no changes were noted between time-points 3 and 4 .

Table 4-15. Results of ELISA for sRAGE at time-points $1 \& 2$.

\begin{tabular}{lll}
\hline & HF & CD \\
\hline $\begin{array}{l}\text { RAGE (beginning of } \\
\text { study, ng/mL) }\end{array}$ & $0.57 \pm 0.16$ & $0.58 \pm 0.11$ \\
$\begin{array}{l}\text { RAGE (after } 3 \text { months of } \\
\text { diet, ng/mL) }\end{array}$ & $0.73 \pm 0.25$ & $0.67 \pm 0.20$ \\
\hline $\begin{array}{l}\text { Values reported as mean } \pm S D . \\
\end{array}$ &
\end{tabular}

Table 4-16. Results of ELISA for sRAGE for surgical groups at all time-points.

\begin{tabular}{lllll}
\hline & HF+OVX & HF+SHAM & CD+OVX & CD+SHAM \\
\hline $\begin{array}{l}\text { RAGE (beginning of } \\
\text { study, ng/mL) }\end{array}$ & $0.65 \pm 0.22$ & $0.51 \pm 0.09$ & $0.59 \pm 0.13$ & $0.59 \pm 0.11$ \\
$\begin{array}{l}\text { RAGE }(\mathbf{a f t e r} \mathbf{3} \text { months } \\
\text { of diet, ng/mL) }\end{array}$ & $0.88 \pm 0.19$ & $0.72 \pm 0.27$ & $0.69 \pm 0.15$ & $0.82 \pm 0.07$ \\
$\begin{array}{l}\text { RAGE }(\mathbf{1} \mathbf{m o n t h} \text { after } \\
\text { surgery, } \mathbf{n g} / \mathbf{m L})\end{array}$ & $0.71 \pm 0.06^{*}$ & $0.52 \pm 0.24$ & $0.71 \pm 0.13 *$ & $0.56 \pm 0.27$ \\
$\begin{array}{l}\text { RAGE (3 months after } \\
\text { surgery, ng/mL) }\end{array}$ & $0.58 \pm 0.25$ & $0.50 \pm 0.27$ & $0.73 \pm 0.21$ & $0.56 \pm 0.22$ \\
\hline $\begin{array}{l}\text { Values reported as mean } \pm S D . \\
\text { * Significant }(p<0.05) \text { compared to SHAM controls. }\end{array}$ &
\end{tabular}




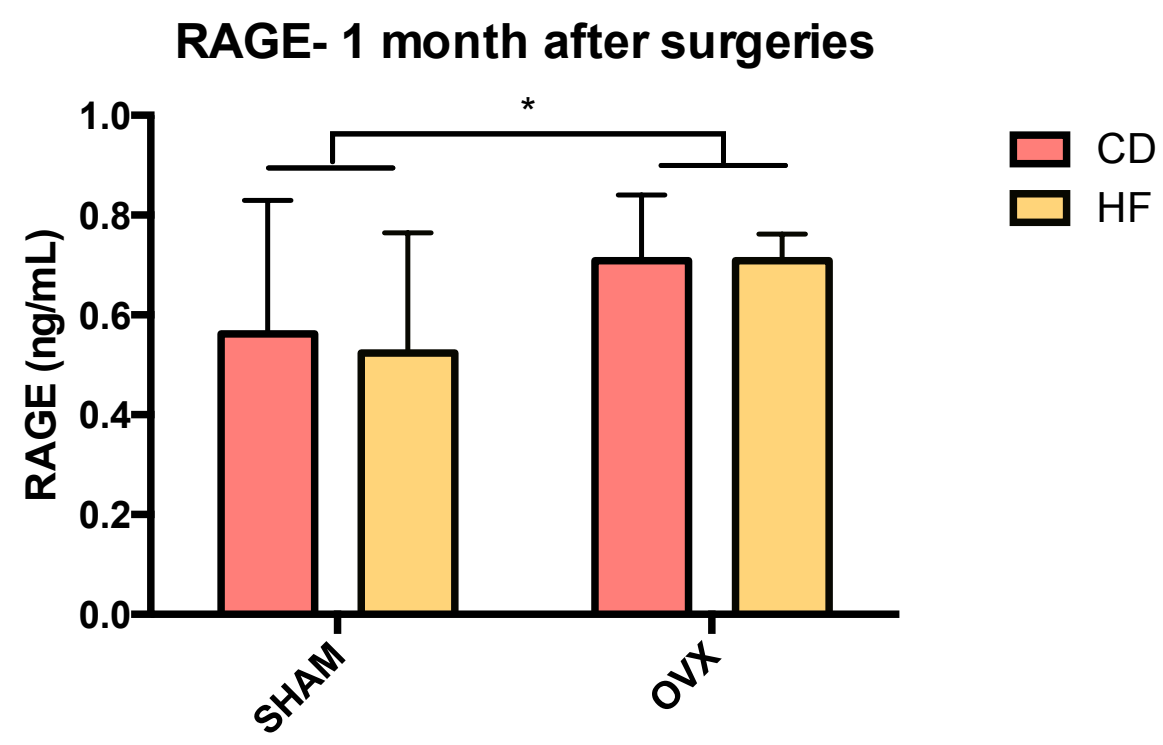

Fig 4-17. ELISA results for soluble RAGE measured one month after surgeries. Two-way ANOVA, mean $\pm \mathrm{SD}, \mathrm{n}=9$. *SHAM significantly different then OVX surgical groups. Diet alone $\mathrm{p}=0.767$, surgery alone $\mathrm{p}=0.015$, interaction $\mathrm{p}=0.767$.

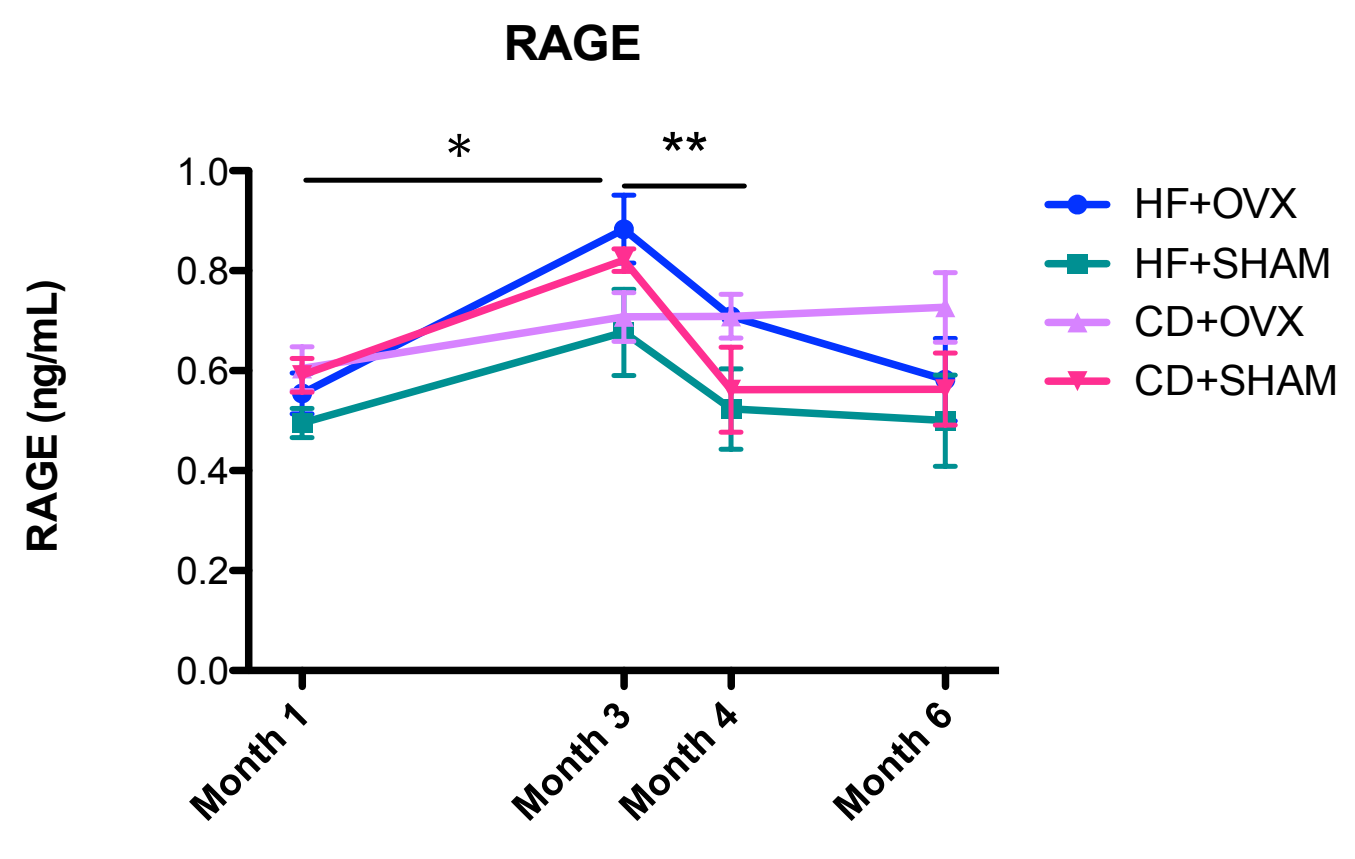

Figure 4-18. ELISA results for sRAGE at each time-point for surgical groups. Paired TTest, mean \pm SD. * Significant increase in sRAGE for all groups $(\mathrm{p}<0.05)$;** significant decrease in $s$ RAGE for HF+OVX ( $p=0.043), H F+$ SHAM $(p=0.042)$ and CD+SHAM $(p=0.015)$. 


\subsection{PROTEIN CARBONYLATION ASSAY}

Carbonylation of bone collagen was investigated as a marker for oxidative damage to collagen molecules. In this assay, bone collagen was analyzed in two fractions; the insoluble fraction was approximately $67 \%$ of the total bone collagen and the soluble fraction was approximately $33 \%$ of the total bone collagen, by volume.

The insoluble fraction and soluble fraction carbonylation were not different between the baseline groups (Table 4-17). When both the fractions of bone collagen taken from the surgical groups were analyzed together, the total carbonyl content showed no detectable differences between the groups due to diet, surgery or an interaction of the two. When the two fractions were analyzed separately, no detectable differences were noted in the soluble fraction, however there was a difference in the insoluble fraction. A two-way ANOVA showed that groups that underwent the OVX surgery had significantly more carbonylation in the insoluble fraction, while diet and the interaction of the two factors did not cause a detectable difference (Table 4-18 \& Figure 4-19)

Table 4-17. Protein carbonylation assay results for $1^{\text {st }}-3^{\text {rd }}$ lumbar vertebrae of baseline groups.

\begin{tabular}{|c|c|c|}
\hline & HFB & CDB \\
\hline $\begin{array}{l}\text { Total Soluble Fraction } \\
(\mu \mathrm{moles} / \mathrm{mg})\end{array}$ & $0.06 \pm 0.02$ & $0.08 \pm 0.03$ \\
\hline $\begin{array}{l}\text { Insoluble Fraction } \\
\text { ( } \mu \text { moles/mg) }\end{array}$ & $0.14 \pm 0.02$ & $0.15 \pm 0.03$ \\
\hline Total Carbonyl ( $\mu$ moles/mg) & $0.20 \pm 0.03$ & $0.22 \pm 0.03$ \\
\hline
\end{tabular}


Table 4-18. Protein carbonylation assay results for $1^{\text {st }}-3^{\text {rd }}$ lumbar vertebrae.

\begin{tabular}{|c|c|c|c|c|}
\hline & $\mathrm{HF}+\mathrm{OVX}$ & HF+SHAM & CD+OVX & CD+SHAM \\
\hline $\begin{array}{l}\text { Total Soluble } \\
\text { Fraction } \\
\text { ( } \mu \text { moles/mg) }\end{array}$ & $0.07 \pm 0.02$ & $0.07 \pm 0.02$ & $0.07 \pm 0.02$ & $0.08 \pm 0.04$ \\
\hline $\begin{array}{l}\text { Insoluble } \\
\text { Fraction } \\
\text { ( } \mu \text { moles/mg) }\end{array}$ & $0.14 \pm 0.02 *$ & $0.14 \pm 0.02$ & $0.15 \pm 0.02 *$ & $0.12 \pm 0.02$ \\
\hline $\begin{array}{l}\text { Total Carbonyl } \\
(\mu \mathrm{moles} / \mathrm{mg})\end{array}$ & $0.21 \pm 0.03$ & $0.21 \pm 0.03$ & $0.22 \pm 0.03$ & $0.20 \pm 0.04$ \\
\hline
\end{tabular}

Values reported as mean $\pm S D$. * Significant $(p<0.05)$ compared to SHAM controls.

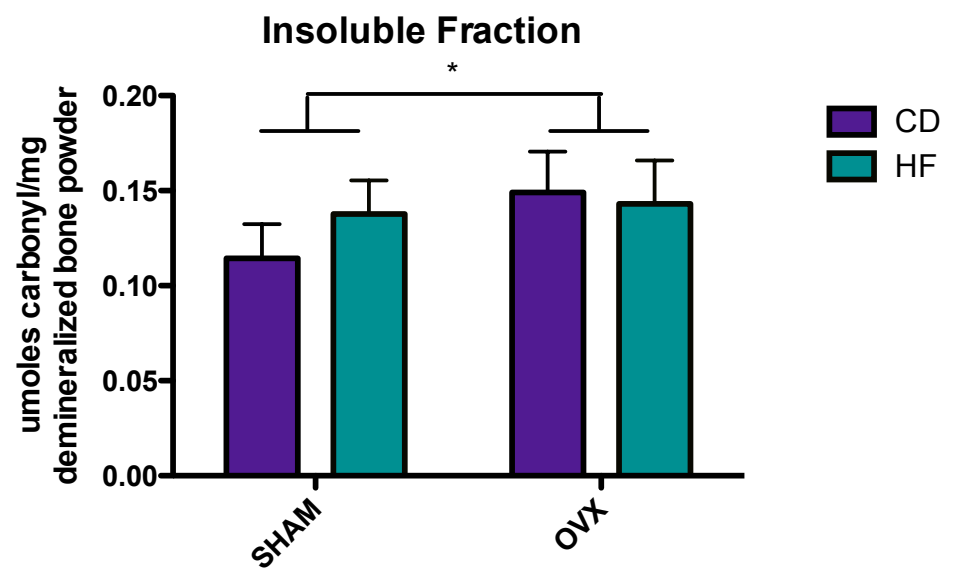

Fig 4-19. Carbonyl assay results for insoluble fraction. Two-way ANOVA, mean $\pm \mathrm{SD}$, $\mathrm{n}=9$. *SHAM significantly different from OVX surgical groups. Diet alone $\mathrm{p}=0.220$, surgery alone $\mathrm{p}=0.007$, interaction $\mathrm{p}=0.042$.

\subsection{HPLC}

HPLC was used to measure pentosidine and mature enzymatic crosslinks (deoxypyridinoline and pyridinoline) in acid hydrolysates of the $4^{\text {th }}$ lumbar vertebrae. No detectable differences in any of the enzymatic crosslinks were noted between the baseline groups (Table 4-19). In the surgical groups, there were no differences in expression of pentosidine or pyridinoline crosslinks. However, a two-way ANOVA showed that 
deoxypyridinoline levels were increased in rats that underwent OVX, while diet and the interaction of the two factors did not have an effect (Table 4-20 \& Figure 4-20).

Table 4-19. HPLC results for L4 vertebrae of baseline groups.

\begin{tabular}{lll}
\hline & HFB & CDB \\
\hline $\begin{array}{l}\text { Pentosidine (pmoles/nmoles } \\
\text { collagen) }\end{array}$ & $0.26 \pm 0.06$ & $0.23 \pm 0.06$ \\
$\begin{array}{l}\text { Pyridinoline (nmoles/nmoles } \\
\text { collagen) }\end{array}$ & $0.11 \pm 0.01$ & $0.12 \pm 0.01$ \\
$\begin{array}{l}\text { Deoxypyridinoline } \\
\text { (nmoles/nmoles collagen) }\end{array}$ & $0.19 \pm 0.02$ & $0.21 \pm 0.02$ \\
\hline
\end{tabular}

Values reported as mean $\pm S D$.

Table 4-20. HPLC results for L4 of surgical groups.

\begin{tabular}{lllll}
\hline & HF+OVX & HF+SHAM & CD+OVX & CD+SHAM \\
\hline $\begin{array}{l}\text { Pentosidine } \\
\text { (pmoles/nmoles } \\
\text { collagen) }\end{array}$ & $0.27 \pm 0.07$ & $0.24 \pm 0.03$ & $0.23 \pm 0.03$ & $0.23 \pm 0.06$ \\
$\begin{array}{l}\text { Pyridinoline } \\
\text { (nmoles/nmoles }\end{array}$ & $0.11 \pm 0.01$ & $0.12 \pm 0.01$ & $0.11 \pm 0.01$ & $0.12 \pm 0.02$ \\
$\begin{array}{l}\text { collagen) } \\
\begin{array}{l}\text { Deoxypyridinoline } \\
\text { (nmoles/nmoles } \\
\text { collagen) }\end{array}\end{array}$ & $0.23 \pm 0.02 *$ & $0.19 \pm 0.02$ & $0.22 \pm 0.03^{*}$ & $0.20 \pm 0.04$ \\
\hline
\end{tabular}

Values reported as mean $\pm S D$. * Significant $(p<0.05)$ compared to SHAM controls. 


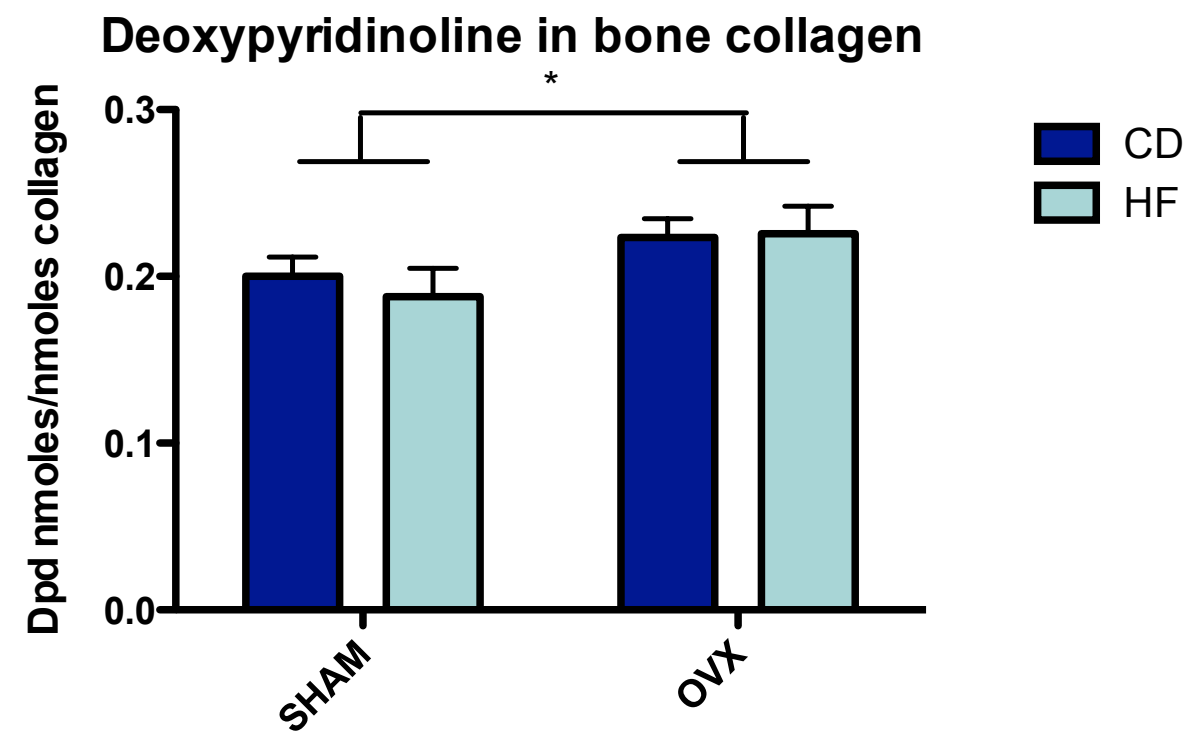

Fig 4-20. HPLC results for bone collagen deoxypyridinoline Two-way ANOVA, mean \pm $\mathrm{SD}, \mathrm{n}=9$. SHAM significantly different form OVX surgical groups. Diet alone $\mathrm{p}=0.590$, surgery alone $\mathrm{p}=0.002$, interaction $\mathrm{p}=0.438$.

\subsection{IMMUNOHISTOCHEMISTRY}

Immunohistochemistry combined with TRAP analysis was performed to determine if TRAP positive osteoclasts were co-localized to RAGE expression. Two controls were used: an istotype control and a negative control, controlling for non-specific primary and secondary antibody staining, respectively (Figure 4-21).

There were no differences between baseline groups or surgical groups for both Oc.RAGE/BS and Oc.RAGE/N.OC (Table 4-21 and 4-22). However, the HFD alone caused a trend for more Oc.RAGE/N.Oc $(\mathrm{p}=0.08)$, while surgery alone or an interaction of diet and surgery did not have any effect. 
Table 4-21. TRAP/RAGE co-localization results for baseline groups.

\begin{tabular}{lll}
\hline & HFB & CDB \\
\hline $\begin{array}{l}\text { Osteoclasts (\#) } \\
\begin{array}{l}\text { RAGE expressing } \\
\text { osteoclasts (\#) }\end{array}\end{array}$ & $85.8 \pm 26.72$ & $143.8 \pm 75.67$ \\
$\begin{array}{l}\text { RAGE expressing } \\
\text { osteoclasts/bone surface } \\
\text { (1/mm) }\end{array}$ & $0.42 \pm 0.11$ & $38 \pm 13.95$ \\
$\begin{array}{l}\text { RAGE expressing } \\
\text { osteoclasts/ total number of } \\
\text { osteoclasts (\%) }\end{array}$ & $0.32 \pm 0.06$ & $0.53 \pm 0.26$ \\
\hline $\begin{array}{l}\text { Values reported as mean } \pm S D . \\
\end{array}$ & \\
\hline
\end{tabular}

Table 4-22. TRAP/RAGE co-localization results for surgical groups.

\begin{tabular}{|c|c|c|c|c|}
\hline & HF+OVX & HF+SHAM & CD+OVX & CD+SHAM \\
\hline Osteoclasts (\#) & $49.88 \pm 26.35$ & $87.5 \pm 44.64$ & $56.63 \pm 48.55$ & $147.1 \pm 94.12$ \\
\hline $\begin{array}{l}\text { RAGE expressing } \\
\text { osteoclasts (\#) }\end{array}$ & $15.5 \pm 11.66$ & $23 \pm 13.27$ & $10.38 \pm 7.6$ & $33.38 \pm 21.21$ \\
\hline $\begin{array}{l}\text { RAGE expressing } \\
\text { osteoclasts/bone surface } \\
(1 / \mathrm{mm})\end{array}$ & $0.52 \pm 0.15$ & $0.46 \pm 0.23$ & $0.34 \pm 0.13$ & $0.47 \pm 0.21$ \\
\hline $\begin{array}{l}\text { RAGE expressing } \\
\text { osteoclasts/ total number } \\
\text { of osteoclasts }(\%)\end{array}$ & $0.29 \pm 0.12^{*}$ & $0.26 \pm 0.05^{*}$ & $0.22 \pm 0.1$ & $0.22 \pm 0.07$ \\
\hline
\end{tabular}

Values reported as mean $\pm S D .{ }^{*}$ Trend $(p=0.08)$ HFD having a greater percentage.

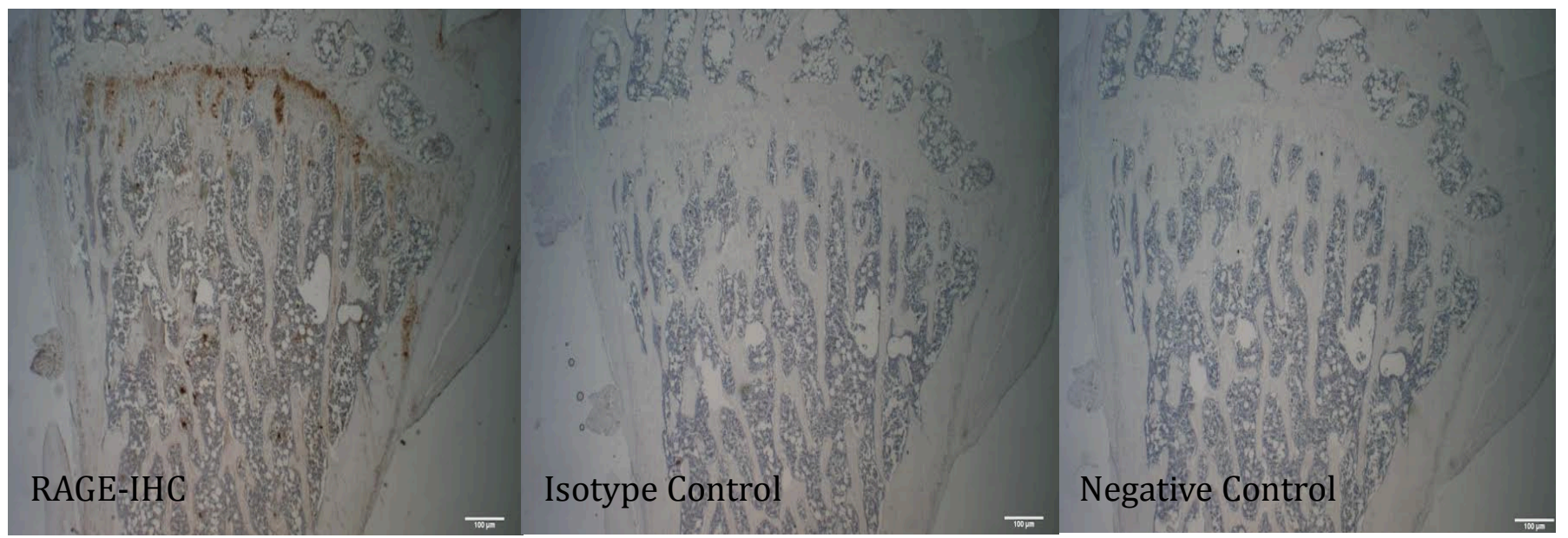

Figure 4-21. Representative images from immunohistochemistry stain, showing sequential sections stained immunohistochemically for RAGE (left) and the two controls used. Istoype control (centre) controls for non-specific primary antibody staining, negative control (right) controls for non-specific secondary antibody staining. 


\section{DISCUSSION}

\subsection{STUDY OBJECTIVES}

The first goal of the present study was to investigate the effects of increased expression of AGEs on bone quality and remodeling in the rat model of postmenopausal bone loss. AGEs are being investigated as possible markers of postmenopausal osteoporosis as an increase in two AGEs, pentosidine and CML, have been found to be elevated in serum of patients with postmenopausal osteoporosis ${ }^{133}$. AGEs have also been found to be accumulated in aged bone, while having a negative correlation with bone density and a positive correlation with the Singh index of osteoporosis severity ${ }^{55}$. Therefore the effect of an accumulation of AGEs on bone loss of the OVX rat model was attempted utilizing a HFD to stimulate glycation.

The second goal of the present study was to determine if the loss of estrogen led to an increased expression of RAGE. RAGE can be activated by many ligands, including AGEs and other products of inflammation including HMGB1 And S100/calgradulins ${ }^{68}$. As the loss of estrogen has been shown to cause an increase in oxidative stress, ${ }^{49,64}$ it may increase the factors that ligate RAGE, subsequently upregulating the expression of the receptor through the positive feedback mechanism caused by the release of NF- $\mathrm{KB}^{58}$. Therefore, to investigate if oxidative stress can cause an increased expression of RAGE, OVX surgeries were performed, and subsequent studies evaluating oxidative stress and RAGE expression were performed.

This discussion is divided into two main components that serve to explain the findings from both objectives of this study. Objective 1) To determine if a high amount of 
bone loss is correlated with elevated bone AGE content and Objective 2) To determine if the loss of estrogen resulting from menopause leads to an increased expression of RAGE.

\subsection{OBJECTIVE 1: EFFECT OF AGES ON BONE LOSS IN OVX}

The effect of an accumulation of AGEs on bone loss of the OVX rat model was attempted utilizing a HFD to stimulate glycation. However, the HFD did not increase AGEs to significant levels in the serum or bone collagen. The majority of changes to bone quality were due to OVX alone and were consistent with previously published measurements.

\subsubsection{MODELS USED}

The current study employed a HFD to increase the production of AGEs in combination with the OVX rat model of postmenopausal osteopenia to investigate bone loss associated with an accumulation of AGEs.

\subsubsection{HIGH FRUCTOSE DIET}

The high fructose diet is commonly used to induce AGE formation, as their creation is dependent on reducing sugar concentrations ${ }^{91,93,134}$. Therefore, theoretically with more fructose present, there will be an increased probability of spontaneous nonenzymatic reactions between reducing sugars and proteins. As fructose has been shown to be the most potent initiator of the Maillard reaction, it was used in this study ${ }^{62}$. High fructose diets have also been shown to cause an elevation in oxidative stress through the production of reactive oxygen species ${ }^{94,95}$. 


\subsubsection{THE OVX MODEL}

The OVX rat model of postmenopausal bone loss is a well-established model for postmenopausal osteopenia, and was employed in the current study to investigate bone loss in conjunction with AGE expression. OVX closely mimics aspects of postmenopausal osteoporosis including increased bone turnover, with resorption exceeding formation leading to a net loss of bone, and a greater loss of trabecular bone

than cortical bone ${ }^{87,88}$. Wronski et al. ${ }^{88,120}$ have shown in several studies that following an OVX procedure rats undergo two phases. The first is rapid bone loss that occurs up to 100 days post-OVX; rats in this stage experience the majority of trabecular bone loss. The second stage is the steady state of bone loss, in which trabecular bone loss is stabilized and bone turnover subsides, although remains higher then SHAM-operated rats. In this study the rats underwent OVX at 3 months of age, and were allowed to lose bone for 3 months. Therefore, at time of sacrifice our rats were at the tail end of the rapid phase of bone loss.

\subsubsection{GROSS EFFECTS OF DIETS AND SURGERIES}

Rat weights were recorded throughout the study. A difference was not observed in the amount of weight gained before the surgeries. Rats on both the HFD and CD seemed to gain weight similarly during the 3 month feeding period. This is consistent with similar reports that used both fructose through the water source ${ }^{62,135}$ and fructose in the $\operatorname{diet}^{92,136}$. However, after the surgeries were performed, it became evident that OVX rats gained significantly more weight then SHAM rats, which is a common phenomenon ${ }^{88,137,138}$. OVX rats have been found previously to have increased food intake and lethargy, ${ }^{137}$ 
however the weight gain is most likely related to estrogen deficiency, as estrogen helps to regulate food intake via the central nervous system ${ }^{139}$. Estrogen is thought to increase vagal cholecystokinin (CCK) peptide, which provides the feeling of satiation after food intake, so when its estrogen production is removed there is a decrease in CCK release ${ }^{139}$. Future support for this theory is seen when estradiol replacement treatment prevents the weight gain seen in $\mathrm{OVX}^{137,139}$.

Sections of livers from each group were also examined for any signs of development of non-alcoholic fatty liver disease (NAFLD). NAFLD has been commonly reported in rats fed a high fructose diet due to the increase of triacylglycerol synthesis in the livers, leading to steatosis, inflammation and fibrosis ${ }^{99,100,127}$. We examined the livers of rats in both the baseline and surgical groups, and after initial gross assessment, signs of NAFLD were not obviously present in any of the groups. This was unexpected as high fructose diets are often used to model human NAFLD in rats, ${ }^{99}$ and high fructose diets have been shown to induce this disease after being fed for a shorter period of time then was used in this study ${ }^{100,127}$. This suggests that the HFD may not have been metabolized to the same effect as in other studies.

\subsubsection{DIET EFFECTS ON AGE PRODUCTION}

To measure AGE production caused by the HF diet, ELISA and HPLC testing was performed on serum and bone samples, respectively.

Serum taken from the rats at time-points 1 and 2 were grouped together as either HFD fed or CD fed, as blood samples were taken before surgical intervention. ELISA results evaluating the levels of circulating pentosidine and CML at these time-points 
showed there were no differences in the levels of each factor between the two diets. This was an interesting and unexpected result, as several other studies using high fructose diets saw significant increases in AGEs after feeding times of just $30{ }^{92}$ and 45 days ${ }^{91}$. However, these studies looked at AGE-related fluorescence of collagen and glycation markers indirectly measuring AGEs, ${ }^{62,91,92,140}$ and did not analyze the serum for specific AGEs using ELISA techniques. Therefore, it is plausible that high fructose diets do not cause a significant increase in serum CML or pentosidine, which is reflected in our results, but instead causes an overall increase in glycation, which is reflected in most literature. It is also plausible that we did not see a difference because our control diet contained $10 \%$ sucrose, which could have also stimulated production of AGEs ${ }^{62}$. Several of the studies did not include any fructose or fructose substitutes in their control diets, $62,91,92,140$ which could explain why we did not see significant differences in our study. This is further supported by the observation that all groups saw an increase in both pentosidine and CML from time-point 1 to time-point 2, regardless of which diet they were placed on.

HPLC analysis was performed to measure pentosidine present in bone collagen, as well as two mature enzymatic collagen cross-links: pyridinoline and deoxypyridinoline. Similar to the ELISA results, HPLC performed on the bones from the baseline groups showed that there was no difference in pentosidine levels. Again, this was surprising as a study that supplied fructose through the water source for a shorter period of time saw an increase in pentosidine levels through HPLC testing ${ }^{61}$. This again supports the speculation that the CD may have had a similar effect to the HFD because it too contained reducing sugars, which may have driven the production of pentosidine. Similarly, pyridinoline and deoxypyridinoline were also unchanged between the two diet groups, 
which what was expected as their production is not controlled by the glycation of collagen, but through enzymatically derived reactions ${ }^{117}$.

\subsubsection{DIET AND SURGERY EFFECTS ON AGE PRODUCTION}

Further analysis at time-points 3 and 4, taken after surgical intervention compared the groups based on the combination of the diet and surgery treatments they received. For pentosidine there continued to be no difference at both time-points 3 and 4 between any of the groups. Again, this was a surprising result because it has been shown many times that patients with osteoporosis have elevated levels of serum pentosidine ${ }^{55,133}$. There was an elevated amount of CML in the rats fed the $\mathrm{CD}$ at time-point 3, with no difference noted at time-point 4. This was also an unexpected result, as based on results from other groups, the HF and OVX surgery were expected to have an effect on serum CML levels 133.

When the rats were taken off of their diets after the surgical procedures it was interesting to see that the levels of pentosidine and CML seemed to plateau and decrease, although it was not a significant decrease for all groups. This suggests that both diets may have been stimulating the production of the AGEs, and when taken off of the diet, there was nothing driving the Maillard reaction forward, leading to a decrease in the amount of circulating AGEs. The CD may have had a stronger effect at prolonging the increased expression of CML after the rats were taken off of the diets, however 3 months after administration of the diets, all groups were at similar levels of expression of CML.

HPLC analysis performed on the surgical groups showed a similar profile. Again, pentosidine levels were unchanged between any of the groups. This could be explained by 
a wearing off of the effect of the diets on the production of pentosidine as seen in the ELISAs, since HPLC analysis was done at the end of the study. However, this result goes against many studies that show increases in bone pentosidine in human patients ${ }^{55}$ with osteoporosis as well as animal models of postmenopausal osteopenia ${ }^{141,142}$. Again, since bone pentosidine was measured at the end of the study, the effects of the OVX surgery may have worn off similarly to what happened in the ELISAs. There may have been an increase in the amount of pentosidine developed due to OVX immediately after the surgeries in the rapid phase of bone loss, but it may have decreased back to baseline levels reaching the steady state of bone loss. Unfortunately, we did not have a time point one month after surgeries to track this. It is also interesting to note that the amount of pentosidine measured at the end of the study, after the rats had undergone surgeries and aged to 9 months, was consistent with the levels measured from the baseline groups. This further suggests that if any changes were seen due to the OVX surgery, the effect was lost by the end of the study.

Pyridinoline levels were also unchanged between the four surgical groups, while deoxypyridinoline levels were significantly increased due to the OVX surgery alone. This was unsurprising for pyridinoline, as the level of the crosslink has been found to be consistent between SHAM and OVX-operated rats previously ${ }^{143}$. However, the increase in deoxypyridinoline levels in OVX-treated rats was unexpected, as it has been found to be either unchanged from SHAM groups ${ }^{143}$ or significantly decreased due to OVX treatment ${ }^{117}$. A theory proposed by Saito et al. ${ }^{141}$ states that accumulation of AGEs may be one of the factors that is causing a reduction in enzymatic cross-links. This is based off the fact that both of these crosslinks on the same essential sites, ${ }^{117}$ and an increase in AGE 
crosslinks accompanied by a decrease in enzymatic crosslinks was found in osteoporotic bone ${ }^{141}$. Therefore, it is plausible that the absence of an increase in pentosidine crosslinks in the OVX groups, lead to an increased level of deoxypyridinoline observed in this study.

These results taken together seem to suggest that both of the diets stimulated the production of serum CML and pentosidine and bone pentosidine similarly in the baseline groups. This could be due to the fact that the CD contains $10 \%$ sucrose, which can also initiate the Maillard reaction ${ }^{62}$. Therefore, both diets could be causing an increase in AGEs to the same extent or the HFD simply did not cause an increase in AGE production. ELISA results showing that both diets stimulated an increase in serum CML and pentosidine, which was later dissipated when the rats were taken off the diets, suggests the first scenario may have occurred. Following the surgeries, any effect of the diets was minimal which is evident through the decreasing levels of both serum CML and pentosidine in the ELISA assays. Interestingly, the OVX surgery did not cause any increase in circulating AGEs, even one month after the surgeries nor at the level of bone pentosidine. In conclusion there did not seem to be an accumulation of AGEs due to the diets or as a result of the surgeries.

\subsubsection{DIET EFFECTS ON BONE QUALITY}

The HFD alone, as measured through analysis of the baseline groups, did not have any effect on any macroscopic (DXA, microCT) or tissue-level (decalcified and undecalcified histomorphometry) skeletal changes as compared to the CD. This is consistent with a study performed by Tsanzi et al., where they fed rats high fructose drink 
and did not observe any differences from the control group in terms of BMD or BMC as measured by DXA, or bone turnover factors such as osteoclacin or alkaline phosphatse 135. Therefore, based on the results of our study and the study by Tsanzi et al., it is likely that high fructose feeding does not have an effect on macroscopic skeletal changes. A similar study investigating the effects of a high fructose diet on tissue level turnover was not found, although a study by Bass et al., looked at the changes in histomorphometry of trabecular bone in rats fed glucose and fructose diets ${ }^{144}$. They found that the rats on the high fructose diet had stronger bones and enhanced trabecular microarchitecture compared to the high glucose fed rats, suggesting that the high fructose diet had an anabolic effect on bone growth. However, this study did not include a comparison to rats on a control diet without the presence of any sugars, so it unclear whether these parameters were changed from baseline values of bone growth. Therefore, the results of the current study seem to suggest that the HFD did not have an effect on tissue level skeletal changes, as compared to the CD.

\subsubsection{DIET AND SURGERY EFFECTS ON BONE QUALITY}

\subsubsection{MACROSCOPIC CHANGES}

As neither the diet nor the surgeries led to an increase in AGE accumulation, the majority of the macroscopic and tissue-level skeletal changes were caused by the OVX surgery alone. The OVX surgeries led to no detectable changes in cortical bone microarchitecture as measured by microCT analysis of the femurs. This is consistent with models of OVX, as cortical bone is affected much slower then trabecular bone, and is more resistant to bone loss following OVX, with the earliest changes recorded between 
90-120 days post-OVX ${ }^{87,89,90}$.

However, OVX did lead to interesting results in BMD. DXA analysis of the femurs showed that there was a significant increase in aBMD for SHAM-treated group, with the interaction of $\mathrm{CD}+\mathrm{SHAM}$ possessing the largest aBMD. While microCT analysis of the femurs showed that OVX-treated groups led to an increase in vBMD. This is an interesting observance that shows the reliability of the two assays. DXA only takes into account the 2D measurements, therefore is unable to detect the depth of a sample, and longer and wider bones will have a greater aBMD ${ }^{36}$. While in microCT, a true representation of the BMD is expressed because this technology is able to perceive and analyze the depth of the sample ${ }^{106}$. Therefore, the true BMD is most likely to be the vBMD received from microCT analysis. While this may seem like an odd result, previous studies have shown that OVX has led to an increase in periosteal bone formation in the cortical diaphysis as compared to SHAM operated controls ${ }^{145,146}$. Although not significant, there was a trend towards OVX surgical groups having a greater cortical thickness, which suggests that there may have been heightened periosteal bone growth in the OVX groups that could have lead to the significant increase in vBMD observed. Since periosteal bone growth would increase the depth of the sample, DXA scanning did not pick it up.

In addition to this, we saw several skeletal changes in the L6 vertebrae of OVX rats through microCT analysis. The OVX surgery led to decreased trabecular bone volume, trabecular number, and trabecular vBMD. This was supported by decreased aBMD for OVX-treated groups observed through DXA analysis. These changes are attributed to the surgery alone, as no differences were detected due to the diets. This is 
consistent with previous reports of OVX induced trabecular bone loss, which has been reported to increase the depth of resorption cavities ${ }^{87,147}$. This perforative resorption leads to the loss of whole trabecular elements as opposed to trabecular thinning, which is seen through the changes in the microarchitecture noted in this study. There was a significant decrease in number of trabecuale as opposed to a decrease in thickness or separation of the trabeculae, and these changes were further translated into significantly decreased trabecular bone volume and density.

\subsubsection{TISSUE-LEVEL BONE REMODELING}

To investigate tissue-level bone remodeling, we employed several histomorphological stains on the proximal tibial metaphysis trabecular bone to determine whether the surgeries and diets had an effect on tissue-level bone turnover. Results showed that OVX surgeries alone led to significant increases in dynamic parameters of bone formation including double label signalling, interlabel width, percent mineralizing surface, mineral apposition rate and bone formation rate normalized to both bone surface and volume. Furthermore, static bone formation parameters including percent osteoid surface and percent osteoid volume were increased due to OVX surgeries alone. Together, this data suggests that OVX significantly increased tissue-level bone formation, consistent with previous results of histomorphometry measuring bone formation in OVX $\operatorname{rats}^{88,121,148,149}$.

An interesting result received from the static histomorphometry was that the HFD alone caused a significant increase in the width of osteoid seam measured in this assay. Past studies have suggested that osteoid width may be correlated with osteoblast activity 
150. Therefore, the increase in osteoid width in the current study suggests that there may have been increased osteoblast activity in groups fed the HFD. This is in line with the conclusions made by Bass et al., who suggest that a high fructose diet may have an anabolic effect ${ }^{144}$. However, since this is the only bone formation parameter that showed changes due to the HFD in the current study, it is unlikely that the diet caused a dramatic increase in osteoblast activity.

Significant changes were not detected in bone resorption parameters, however several trends were shown. Firstly, the interaction of the HFD and OVX surgery showed a trend of having increased percent osteoclast surface and number of osteoclasts per bone surface. In a study conducted by Wronski et al. ${ }^{88}$ they found that OVX rats undergo a period of rapid bone loss, which increases osteoclastic surface for the first 100 days after surgeries, then experience a steady state of bone loss. In our studies we allowed our rats to lose bone for 3 months, so we may have missed the period where osteoclasts were most active, and thus expressing the most amount of TRAP. This is clearly evident in the CD+OVX group, whose percent osteoclast surface and number of osteoclasts/ bone surface are similar, if not less then, the SHAM-operated groups. However, in the $\mathrm{HF}+\mathrm{OVX}$, this decrease is not experienced and the levels of both these parameters stay elevated, possibly suggesting that the HFD in combination with the OVX surgery is having some sort of preventative effect against a decrease in the activity of the osteoclasts.

This trend mimics the findings by Hanayama et al., a group who also investigated the effects of a high fructose diet on OVX treated rats ${ }^{151}$. When this group performed TRAP staining they saw that OVX, high fructose fed rats had a significantly higher TRAP 
positive area of the proximal tibia, as compared to OVX, control diet fed rats. However, in this study the rats were sacrificed 4 weeks after OVX, so the high fructose diet may not have had a protective effect, but may have just increased the amount of active osteoclasts. They also saw decrease in the BMD measured by DXA in the OVX, high fructose group as compared to the OVX, control diet fed group. It would be interesting to see if the same effects occurred in our study, but we may just have missed them as we allowed our rats to approach the steady state of bone loss.

This result is also interesting in the fact that we did not see any changes in AGEs due to the HFD, so the diet may have been working to increase osteoclast activity in a different way then our proposed mechanism. One potential explanation could be an increase in oxidative stress due to the feeding, as HFD have been previously been shown to cause an increase in oxidative stress ${ }^{62,94,95}$. ROS created by oxidative stress have been shown to have an effect on osteoclast differentiation and resorption, both directly and indirectly ${ }^{152}$. Garrett et al., showed that addition of a superoxide-producing enzyme increased osteoclast number and activity ${ }^{153}$, while Yang et al. showed that an inhibitor of NADPH oxidase led to decreased bone resorption ${ }^{154}$. Other studies show that ROS may instead increase the activation of intracellular signalling pathways such as NF- $\mathrm{kB}$, leading to the release of pro-inflammatory cytokines that could control osteoclast differentiation and activity ${ }^{155}$. Hanayama et al. explored this in their study by treating HF+OVX rats with fluvastatin, which they believed worked in part as an antioxidant to cause a significant decrease in the amount of bone loss and TRAP positive surface ${ }^{151}$. 


\subsubsection{OBJECTIVE 1: CONCLUDING SUMMARY}

The goal of the HFD was to characterize the effects of AGEs on bone loss during OVX. It was expected that the HFD would cause an increase in AGEs through initiating the Maillard reaction, which has been observed in previous studies. However, no differences in AGEs were seen between rats fed the HFD and rats fed the CD. There was no difference in circulating pentosidine or CML, or in the amounts of bone collagen bound pentosidine, measured directly after HFD feeding (baseline groups), or 3 months after the diet was discontinued (surgical groups). Therefore, majority of the skeletal changes that were observed were solely due to the OVX treatment, and the results we saw were mostly consistent with previous studies. Macroscopically, we saw that the OVXtreatment lead to more bone loss in trabecular bone while increasing vBMD in cortical bone. At the tissue level, we saw an increase in bone formation parameters, although no significant changes were seen in bone resorption parameters.

\subsection{OBJECTIVE 2: EFFECT OF ESTROGEN LOSS ON EXPRESSION OF RAGE}

To investigate if estrogen deficiency can cause an increased expression of RAGE, OVX surgeries were performed, and subsequent studies evaluating oxidative damage and RAGE expression were performed. The OVX surgery worked to increase the oxidative damage experienced through protein carbonylation. Soluble RAGE seemed to be sustained at higher levels in the OVX groups, while cell-bound RAGE showed a trend of increased expression on osteoclasts in the HFD fed surgical groups. 


\subsubsection{OXIDATIVE DAMAGE}

\subsubsection{DIET EFFECTS ON OXIDATIVE DAMAGE}

Bones from the baseline groups were investigated for oxidative damage through a protein carbonylation assay. We did not see any differences in protein carbonyaltion in the insoluble or soluble fraction for bone collagen in the baseline groups. This suggests that the HFD alone did not cause oxidative stress, as has been seen in previous studies $62,94,95$. This may have been due to oxidative damage not occurring within the bone collagen, as other groups investigated serum or hepatic markers of oxidative stress or carbonylation of liver tissue ${ }^{62,94,95}$. In further examination it would be interesting to see if oxidative damage occurred due to the HFD in other areas of the rat through assays used in previous studies.

\subsubsection{DIET AND SURGERY EFFECTS ON OXIDATIVE DAMAGE}

We also investigated whether the combination of the diets and surgeries had any effect on the level of oxidative damage accumulated in the rat bone collagen. We analyzed both the soluble fraction, containing a small amount of soluble collagen and other proteins that make up the organic phase of the bone, and the insoluble fraction, which typically contains the majority of collagen, as the bonds that stabilize the fibrillar structure typically protect it from dissolution ${ }^{156}$. The soluble fraction did not experience any notable oxidative damage, while the OVX-treated groups had significantly increased oxidatice damage in the insoluble fraction.

This is consistent with previous reports that observed an increase in oxidative stress following $\mathrm{OVX}^{157,158}$. It is possible that this increase was caused by the stoppage of 
estrogen production, which is most likely due to its role as an antioxidant ${ }^{48,50-52}$. Estrogen deficiency has shown to increase levels of oxidative stress in both human and rodent models ${ }^{51,52}$, along with a decrease in levels of thiol antioxidants ${ }^{158}$. As discussed before, oxidative stress leads to an increased differentiation and activity of osteoclasts, so this may be the mechanism behind the increase in bone loss observed in the trabecular bone in L6 ${ }^{40,158}$.

\subsubsection{SOLUBLE RAGE}

Soluble RAGE (sRAGE) exists as a truncated variant of RAGE, created through alternative splicing of RAGE pre-mRNA ${ }^{67}$. Several isoforms have been identified and are thought to act as decoy receptors, competing with cell surface RAGE to blunt intracellular

effects that occur after ligation of the receptor ${ }^{74,76}$. Although this is just a proposed theory behind the role of sRAGE, its tempting to suggest that in times of inflammation or stress, there is a higher expression of sRAGE to help dull the effect of RAGE ligation. Changes in the ratio of sRAGE/RAGE is tissue-specific and may serve as a biomarker for human diseases ${ }^{68,70,76}$.

We measured sRAGE levels through ELISA testing of serum at four time-points throughout the study. At time-points 1 and 2, all rats were grouped as either HFD fed or CD fed, and we did not note any differences in sRAGE between the two diets. However, all groups saw a significant increase in sRAGE levels, regardless of the diet they were placed on. This seems to further support the ideal that both of the diets were having similar effects on the rats, and may have both caused an inflammatory response that spurred the creation of sRAGE. Further analysis at time-points 3 and 4 showed an 
elevated amount of sRAGE in OVX-treated rats one month after surgeries (time-point 3), and no changes between the groups at time-point 4 .

Interestingly, the levels of sRAGE seemed to peak at time-point 2 and decrease in groups $\mathrm{HF}+\mathrm{OVX}, \mathrm{HF}+\mathrm{SHAM}$ and $\mathrm{CD}+\mathrm{SHAM}$ after they were taken off their respective diets. While OVX-treated groups did have elevated amount of sRAGE at time-point 3, this did not seem to be caused by an increase from the surgery, just a higher level of sustained sRAGE. This suggests that the diets alone may have caused an increase in sRAGE levels, and the OVX surgery may have sustained heightened levels of sRAGE but did not work to increase it. This is an interesting result as RAGE has been shown to be increased in times of inflammation and oxidative stress, which would have occurred following OVX; so in theory there would be an elevated sRAGE/RAGE ratio. However, it is possible that the production of sRAGE was increased immediately after the surgery to help blunt the effect of RAGE and then stopped being produced over the month between time-point 2 and 3, leading to higher, but not increased levels of sRAGE in OVX-treated rats.

\subsubsection{CELL-BOUND RAGE}

To further investigate the expression of cell-bound RAGE, we performed an immunohistochemistry assay to study the co-localization of RAGE with TRAPexpressing osteoclasts at the tissue level. Although no statistically significant changes were observed in the amount of osteoclasts expressing RAGE, a trend was seen in which groups fed the HFD had a higher percent of RAGE expressing osteoclasts. This suggests that the HFD may have induced more RAGE expression within the active osteoclasts. We 
can be fairly certain that this trend towards an increase in RAGE is probably not due to accumulation of AGEs from our observations in Objective 1. Therefore, it is possible that this trend towards more RAGE expression was caused by oxidative stress that may have been inflicted by the HFD. The oxidative stress may have led to inflammation causing an increase in other RAGE ligands such as HMGB1 or S100/calgrandulin. Both ligands have been shown to be increased at sites of inflammation ${ }^{68}$, and HMGB1 has been shown to be increased in the cortex of OVX rats ${ }^{159}$, while an increase in S100A16 was found due to estrogen depletion ${ }^{160}$. Ligation of either of these molecules to RAGE could have led to upregulation of the receptor through $\mathrm{NF}-\kappa \mathrm{B}$ expression. Therefore, if there was an increase in one of these factors due to oxidative stress and subsequent inflammation by the HFD, it is possible that RAGE expression could have increased on active osteoclasts leading to the trend of HFD groups having a more RAGE expressing osteoclasts.

This is further supported by examination and comparison of osteoclast RAGE expression in the CD+SHAM and CD+OVX. It is evident that the OVX surgery alone did not cause a detectable difference in the amount of RAGE expressing osteoclasts; therefore this suggests that the HFD and its subsequent effects could be needed to alter osteoclast expression of RAGE.

\subsubsection{OBJECTIVE 2: CONCLUDING SUMMARY}

For this objective we attempted to observe whether RAGE was expressed at a higher level in both its soluble and cell-bound form following OVX-treatment. We found that the OVX-treatment led to more oxidative damage, which may have led to increase in bone loss seen in OVX-treated rats from Objective 1. We found that sRAGE seemed to be 
increased by both the HFD and CD, further leading into the idea in Objective 1 that the diets had the similar effects on the rats. The OVX surgery did not increase the level of sRAGE, but instead seemed to sustain its elevation more than the SHAM surgery. No significant changes were found in cell-bound RAGE expression, although the results suggested that groups fed the HFD had more RAGE expressing osteoclasts, although only a trend was noted at this time.

\subsection{OVERALL CONCLUSIONS}

This thesis attempted to explore the role of AGEs on bone loss following OVX through the ligation of RAGE. The HFD was unsuccessful in causing accumulation of AGEs; therefore we were unable to observe whether more bone loss is seen with increased expression of AGEs, and whether this could be through interaction with RAGE. The OVX surgeries were successful, and we were able to see the skeletal changes associated with this model. Only sRAGE expression was significantly altered, although both the HFD and CD affected it similarly, and the OVX surgeries seemed to sustain the increase caused by the diets. This study exhibited trends toward the HFD having an effect on both active osteoclasts and osteoclast RAGE expression, as both these parameters seemed to be increased, though not significantly, in groups that were fed HFD. These results suggest that the interaction of the HFD with OVX may have caused an increase in osteoclast activity due to oxidative stress caused by the HFD. This may have lead to more RAGE expression and a subsequent increase in the activity of the RAGE expressing osteoclasts. However, since the results were not significant, further study into this subject should be performed before any conclusions are made. 


\subsection{FUTURE STUDIES}

To further evaluate the effects of AGE/RAGE interaction on the development of postmenopausal osteoporosis, several changes could be made to the experimental design.

1) The diets that were given to the rats need to be altered because the accumulation of AGEs due to the HFD was not prevalent within the bones or serum in our HFD groups. This may have been due to the composition of the $\mathrm{CD}$, which contained $10 \%$ sucrose. In other studies the control diet fed did not contain any fructose or fructose substitutes. It is a possibility that we did not see any differences between the groups because the control diet also caused an increase in AGEs. In future studies I would use a control diet that did not contain any fructose or fructose substitutes, similar to the diets used by Nandhini et al., ${ }^{92}$ and Thirunavukkarasu et al., ${ }^{91}$ or administer fructose through the water source with the rats on standard diets, similar to studies by Levi et al., ${ }^{62}$ and Mikulikova et al. ${ }^{61}$.

2) Since the HFD diet may have not caused an increase in AGEs, another method to induce production and accumulation of AGEs could be used. An alternative method is the administration of streptozotocin, which is a chemical that is toxic to beta cells in the pancreas that produce insulin ${ }^{161}$. The use of streptozotocin causes the development of diabetes, which subsequently can cause the products of AGEs, most likely due to oxidative stress and the formation of hyperglycaemia ${ }^{162,163}$. Several studies have used this method to study AGEs and methods of clearing them or stopping their production ${ }^{162-165}$. Another method includes daily subcutaneous injections of galactose or glucose, which can also cause an increase 
in the expression of AGEs; again most likely due to increased oxidative stress and hyperglycaemia ${ }^{166}$.

3) After ceasing the diet, the AGEs found in the serum decreased, so to see the full effect of increased AGEs on bone loss, it would be wise to continue the diet after the surgeries were performed.

4) As seen in TRAP analysis, we may have missed the window where the most bone was being resorbed, and therefore missed the staining of the most active osteoclasts. To combat this, we could sacrifice sooner after the surgeries, or have another control group that is sacrificed one month after surgeries.

5) To further identify the level of oxidative damage or ROS released due to the HFD and OVX, several more experiments could have been performed. These include performing Western blots or ELISAs on various tissues to quantify expression of an oxidative stress biomarker such as hydrogen peroxide.

6) Since RAGE is a multiligand receptor, other factors that could be ligating to RAGE, such as HMGB1 and S100 should also be measured to be able to make distinct conclusions about what is causing an upregulation of RAGE expression. This could be done through Western blots or ELISAs on blood serum or bone tissue samples.

7) As discussed previously, RAGE is also present on many other types of cells including but not limited to monocytes/macrophages, endothelial cells, neuronal cells ${ }^{64}$,and mesenchyal stromal cells ${ }^{69}$. It would be interesting to evaluate whether there was an increase in expression in these cells as well. This could be done through use of immunohistochemistry and/or Western blot assays 
8) Immunohistochemical staining for AGEs could also be performed to allow for microscopic observation of AGEs, and allow for the co-localization of RAGE and AGE expression. 


\section{REFERENCES}

1. Lin JT, Lane JM. Osteoporosis: A review. Clin Orthop Relat Res. 2004;(425)(425):126-134.

2. Bjarnason NH, Hassager C, Christiansen C. Postmenopausal bone remodelling and hormone replacement. Climacteric. 1998;1(1):72-79.

3. Florencio-Silva R, Sasso GR, Sasso-Cerri E, Simoes MJ, Cerri PS. Biology of bone tissue:

Structure, function, and factors that influence bone cells. Biomed Res Int. 2015;2015:421746.

4. Taichman RS. Blood and bone: Two tissues whose fates are intertwined to create the hematopoietic stem-cell niche. Blood. 2005;105(7):2631-2639.

5. Buckwalter JA, Glimcher MJ, Cooper RR, Recker R. Bone biology. I: Structure, blood supply, cells, matrix, and mineralization. Instr Course Lect. 1996;45:371-386.

6. Parfitt AM. Implications of architecture for the pathogenesis and prevention of vertebral fracture. Bone. 1992;13 Suppl 2:S41-7.

7. Rho JY, Kuhn-Spearing L, Zioupos P. Mechanical properties and the hierarchical structure of bone. Med Eng Phys. 1998;20(2):92-102.

8. Clarke B. Normal bone anatomy and physiology. Clin J Am Soc Nephrol. 2008;3 Suppl 3:S1319.

9. Boyle WJ, Simonet WS, Lacey DL. Osteoclast differentiation and activation. Nature. 2003;423(6937):337-342.

10. Teitelbaum SL, Ross FP. Genetic regulation of osteoclast development and function. Nat Rev Genet. 2003;4(8):638-649. 
11. Ross FP, Teitelbaum SL. Alphavbeta3 and macrophage colony-stimulating factor: Partners in osteoclast biology. Immunol Rev. 2005;208:88-105.

12. Vaananen HK, Zhao H, Mulari M, Halleen JM. The cell biology of osteoclast function. $J$ Cell Sci. 2000;113 ( Pt 3)(Pt 3):377-381.

13. Teitelbaum SL, Abu-Amer Y, Ross FP. Molecular mechanisms of bone resorption. J Cell Biochem. 1995;59(1):1-10.

14. Mackie EJ. Osteoblasts: Novel roles in orchestration of skeletal architecture. Int J Biochem Cell Biol. 2003;35(9):1301-1305.

15. Li Y, Backesjo CM, Haldosen LA, Lindgren U. IL-6 receptor expression and IL-6 effects change during osteoblast differentiation. Cytokine. 2008;43(2):165-173.

16. Barhanpurkar AP, Gupta N, Srivastava RK, et al. IL-3 promotes osteoblast differentiation and bone formation in human mesenchymal stem cells. Biochem Biophys Res Commun.

2012;418(4):669-675.

17. Knothe Tate ML, Adamson JR, Tami AE, Bauer TW. The osteocyte. Int J Biochem Cell Biol. 2004;36(1):1-8.

18. Bonewald LF. Establishment and characterization of an osteocyte-like cell line, MLO-Y4. J Bone Miner Metab. 1999;17(1):61-65.

19. Roschger P, Paschalis EP, Fratzl P, Klaushofer K. Bone mineralization density distribution in health and disease. Bone. 2008;42(3):456-466.

20. Grynpas M. Age and disease-related changes in the mineral of bone. Calcif Tissue Int. 1993;53 Suppl 1:S57-64. 
21. Burr DB. Targeted and nontargeted remodeling. Bone. 2002;30(1):2-4.

22. Parfitt AM. Targeted and nontargeted bone remodeling: Relationship to basic multicellular unit origination and progression. Bone. 2002;30(1):5-7.

23. Marie PJ, Kassem M. Extrinsic mechanisms involved in age-related defective bone formation. J Clin Endocrinol Metab. 2011;96(3):600-609.

24. Roodman GD. Cell biology of the osteoclast. Exp Hematol. 1999;27(8):1229-1241.

25. Delaisse JM, Andersen TL, Engsig MT, Henriksen K, Troen T, Blavier L. Matrix metalloproteinases (MMP) and cathepsin K contribute differently to osteoclastic activities. Microsc Res Tech. 2003;61(6):504-513.

26. Eriksen EF. Normal and pathological remodeling of human trabecular bone: Three dimensional reconstruction of the remodeling sequence in normals and in metabolic bone disease. Endocr Rev. 1986;7(4):379-408.

27. Bonewald LF, Mundy GR. Role of transforming growth factor-beta in bone remodeling. Clin Orthop Relat Res. 1990;(250)(250):261-276.

28. Walker EC, McGregor NE, Poulton IJ, et al. Cardiotrophin-1 is an osteoclast-derived stimulus of bone formation required for normal bone remodeling. J Bone Miner Res. 2008;23(12):20252032.

29. Negishi-Koga T, Shinohara M, Komatsu N, et al. Suppression of bone formation by osteoclastic expression of semaphorin 4D. Nat Med. 2011;17(11):1473-1480.

30. Zhao C, Irie N, Takada Y, et al. Bidirectional ephrinB2-EphB4 signaling controls bone homeostasis. Cell Metab. 2006;4(2):111-121. 
31. Anderson HC. Matrix vesicles and calcification. Curr Rheumatol Rep. 2003;5(3):222-226.

32. Robling AG, Castillo AB, Turner $\mathrm{CH}$. Biomechanical and molecular regulation of bone remodeling. Annu Rev Biomed Eng. 2006;8:455-498.

33. Boskey AL, Coleman R. Aging and bone. J Dent Res. 2010;89(12):1333-1348.

34. Melton LJ,3rd. Osteoporosis: Etiology, diagnosis and management. New York, New York: Raven Press; 1988.

35. Mundy GR. Osteoporosis: Pathophysiology and non-pharmacological management. Best Pract Res Clin Rheumatol. 2001;15(5):727-745.

36. Kanis JA. Diagnosis of osteoporosis and assessment of fracture risk. Lancet. 2002;359(9321):1929-1936.

37. Compston J. Mechanisms of bone loss and gain in untreated and treated osteoporosis. Endocrine. 2002;17(1):21-27.

38. Parfitt AM, Villanueva AR, Foldes J, Rao DS. Relations between histologic indices of bone formation: Implications for the pathogenesis of spinal osteoporosis. J Bone Miner Res. 1995;10(3):466-473.

39. Marie PJ. Human osteoblastic cells: A potential tool to assess the etiology of pathologic bone formation. J Bone Miner Res. 1994;9(12):1847-1850.

40. Manolagas SC. From estrogen-centric to aging and oxidative stress: A revised perspective of the pathogenesis of osteoporosis. Endocr Rev. 2010;31(3):266-300. 
41. Almeida M, Han L, Martin-Millan M, et al. Skeletal involution by age-associated oxidative stress and its acceleration by loss of sex steroids. J Biol Chem. 2007;282(37):27285-27297.

42. Manolagas SC. Role of cytokines in bone resorption. Bone. 1995;17(2 Suppl):63S-67S.

43. Zallone A. Direct and indirect estrogen actions on osteoblasts and osteoclasts. Ann N Y Acad Sci. 2006;1068:173-179.

44. Manolagas SC. Birth and death of bone cells: Basic regulatory mechanisms and implications for the pathogenesis and treatment of osteoporosis. Endocr Rev. 2000;21(2):115-137.

45. Hughes DE, Dai A, Tiffee JC, Li HH, Mundy GR, Boyce BF. Estrogen promotes apoptosis of murine osteoclasts mediated by TGF-beta. Nat Med. 1996;2(10):1132-1136.

46. Greendale GA, Edelstein S, Barrett-Connor E. Endogenous sex steroids and bone mineral density in older women and men: The rancho bernardo study. J Bone Miner Res. 1997;12(11):1833-1843.

47. Meryl S. LeBoff, Julia Glowacki. The aging skeleton. 1st ed. San Diego, California: Academic Press; 1999.

48. Signorelli SS, Neri S, Sciacchitano S, et al. Behaviour of some indicators of oxidative stress in postmenopausal and fertile women. Maturitas. 2006;53(1):77-82.

49. Massafra C, Gioia D, De Felice C, et al. Effects of estrogens and androgens on erythrocyte antioxidant superoxide dismutase, catalase and glutathione peroxidase activities during the menstrual cycle. J Endocrinol. 2000;167(3):447-452.

50. Bednarek-Tupikowska G, Bohdanowicz-Pawlak A, Bidzinska B, Milewicz A, AntonowiczJuchniewicz J, Andrzejak R. Serum lipid peroxide levels and erythrocyte glutathione peroxidase 
and superoxide dismutase activity in premenopausal and postmenopausal women. Gynecol Endocrinol. 2001;15(4):298-303.

51. Goettsch C, Babelova A, Trummer O, et al. NADPH oxidase 4 limits bone mass by promoting osteoclastogenesis. J Clin Invest. 2013;123(11):4731-4738.

52. Almeida M, Han L, Ambrogini E, Bartell SM, Manolagas SC. Oxidative stress stimulates apoptosis and activates NF-kappaB in osteoblastic cells via a PKCbeta/p66shc signaling cascade: Counter regulation by estrogens or androgens. Mol Endocrinol. 2010;24(10):2030-2037.

53. Avery NC, Bailey AJ. The effects of the maillard reaction on the physical properties and cell interactions of collagen. Pathol Biol (Paris). 2006;54(7):387-395.

54. Hein GE. Glycation endproducts in osteoporosis--is there a pathophysiologic importance? Clin Chim Acta. 2006;371(1-2):32-36.

55. Odetti P, Rossi S, Monacelli F, et al. Advanced glycation end products and bone loss during aging. Ann N Y Acad Sci. 2005;1043:710-717.

56. Miyata T, Notoya K, Yoshida K, et al. Advanced glycation end products enhance osteoclastinduced bone resorption in cultured mouse unfractionated bone cells and in rats implanted subcutaneously with devitalized bone particles. J Am Soc Nephrol. 1997;8(2):260-270.

57. McCarthy AD, Uemura T, Etcheverry SB, Cortizo AM. Advanced glycation endproducts interefere with integrin-mediated osteoblastic attachment to a type-I collagen matrix. Int $J$ Biochem Cell Biol. 2004;36(5):840-848. 
58. Fleming TH, Humpert PM, Nawroth PP, Bierhaus A. Reactive metabolites and AGE/RAGEmediated cellular dysfunction affect the aging process: A mini-review. Gerontology. 2011;57(5):435-443.

59. Kislinger T, Fu C, Huber B, et al. N(epsilon)-(carboxymethyl)lysine adducts of proteins are ligands for receptor for advanced glycation end products that activate cell signaling pathways and modulate gene expression. J Biol Chem. 1999;274(44):31740-31749.

60. Singh R, Barden A, Mori T, Beilin L. Advanced glycation end-products: A review. Diabetologia. 2001;44(2):129-146.

61. Mikulikova K, Eckhardt A, Kunes J, Zicha J, Miksik I. Advanced glycation end-product pentosidine accumulates in various tissues of rats with high fructose intake. Physiol Res. 2008;57(1):89-94.

62. Levi B, Werman MJ. Long-term fructose consumption accelerates glycation and several agerelated variables in male rats. $J$ Nutr. 1998;128(9):1442-1449.

63. Sell DR, Nagaraj RH, Grandhee SK, et al. Pentosidine: A molecular marker for the cumulative damage to proteins in diabetes, aging, and uremia. Diabetes Metab Rev. 1991;7(4):239-251.

64. Bierhaus A, Hofmann MA, Ziegler R, Nawroth PP. AGEs and their interaction with AGEreceptors in vascular disease and diabetes mellitus. I. the AGE concept. Cardiovasc Res. 1998;37(3):586-600.

65. Hamada Y, Fujii H, Fukagawa M. Role of oxidative stress in diabetic bone disorder. Bone. 2009;45 Suppl 1:S35-8. 
66. Biemel KM, Reihl O, Conrad J, Lederer MO. Formation pathways for lysine-arginine crosslinks derived from hexoses and pentoses by maillard processes: Unraveling the structure of a pentosidine precursor. J Biol Chem. 2001;276(26):23405-23412.

67. Bierhaus A, Humpert PM, Morcos M, et al. Understanding RAGE, the receptor for advanced glycation end products. J Mol Med (Berl). 2005;83(11):876-886.

68. Xie J, Mendez JD, Mendez-Valenzuela V, Aguilar-Hernandez MM. Cellular signalling of the receptor for advanced glycation end products (RAGE). Cell Signal. 2013;25(11):2185-2197.

69. Bucciarelli LG, Wendt T, Rong L, et al. RAGE is a multiligand receptor of the immunoglobulin superfamily: Implications for homeostasis and chronic disease. Cell Mol Life Sci. 2002;59(7):1117-1128.

70. Sparvero LJ, Asafu-Adjei D, Kang R, et al. RAGE (receptor for advanced glycation endproducts), RAGE ligands, and their role in cancer and inflammation. J Transl Med. 2009;7:17$5876-7-17$

71. Zhou Z, Immel D, Xi CX, et al. Regulation of osteoclast function and bone mass by RAGE. $J$ Exp Med. 2006;203(4):1067-1080.

72. McCarthy AD, Etcheverry SB, Cortizo AM. Advanced glycation endproduct-specific receptors in rat and mouse osteoblast-like cells: Regulation with stages of differentiation. Acta Diabetol. 1999;36(1-2):45-52.

73. Kume S, Kato S, Yamagishi S, et al. Advanced glycation end-products attenuate human mesenchymal stem cells and prevent cognate differentiation into adipose tissue, cartilage, and bone. J Bone Miner Res. 2005;20(9):1647-1658. 
74. Malherbe P, Richards JG, Gaillard H, et al. cDNA cloning of a novel secreted isoform of the human receptor for advanced glycation end products and characterization of cells co-expressing cell-surface scavenger receptors and swedish mutant amyloid precursor protein. Brain Res Mol Brain Res. 1999;71(2):159-170.

75. Schlueter C, Hauke S, Flohr AM, Rogalla P, Bullerdiek J. Tissue-specific expression patterns of the RAGE receptor and its soluble forms--a result of regulated alternative splicing? Biochim Biophys Acta. 2003;1630(1):1-6.

76. Yonekura H, Yamamoto Y, Sakurai S, et al. Novel splice variants of the receptor for advanced glycation end-products expressed in human vascular endothelial cells and pericytes, and their putative roles in diabetes-induced vascular injury. Biochem J. 2003;370(Pt 3):1097-1109.

77. Lalla E, Lamster IB, Feit M, et al. Blockade of RAGE suppresses periodontitis-associated bone loss in diabetic mice. J Clin Invest. 2000;105(8):1117-1124.

78. McCarthy AD, Etcheverry SB, Bruzzone L, Lettieri G, Barrio DA, Cortizo AM. Nonenzymatic glycosylation of a type I collagen matrix: Effects on osteoblastic development and oxidative stress. BMC Cell Biol. 2001;2:16.

79. Coughlan MT, Thorburn DR, Penfold SA, et al. RAGE-induced cytosolic ROS promote mitochondrial superoxide generation in diabetes. J Am Soc Nephrol. 2009;20(4):742-752.

80. Miyata T, Kawai R, Taketomi S, Sprague SM. Possible involvement of advanced glycation end-products in bone resorption. Nephrol Dial Transplant. 1996;11 Suppl 5:54-57.

81. Dong XN, Qin A, Xu J, Wang X. In situ accumulation of advanced glycation endproducts (AGEs) in bone matrix and its correlation with osteoclastic bone resorption. Bone.

2011;49(2):174-183. 
82. Ding KH, Wang ZZ, Hamrick MW, et al. Disordered osteoclast formation in RAGE-deficient mouse establishes an essential role for RAGE in diabetes related bone loss. Biochem Biophys Res Commun. 2006;340(4):1091-1097.

83. Stolzing A, Sellers D, Llewelyn O, Scutt A. Diabetes induced changes in rat mesenchymal stem cells. Cells Tissues Organs. 2010;191(6):453-465.

84. Sanguineti R, Storace D, Monacelli F, Federici A, Odetti P. Pentosidine effects on human osteoblasts in vitro. Ann N Y Acad Sci. 2008;1126:166-172.

85. Franke S, Siggelkow H, Wolf G, Hein G. Advanced glycation endproducts influence the mRNA expression of RAGE, RANKL and various osteoblastic genes in human osteoblasts. Arch Physiol Biochem. 2007;113(3):154-161.

86. Alikhani M, Alikhani Z, Boyd C, et al. Advanced glycation end products stimulate osteoblast apoptosis via the MAP kinase and cytosolic apoptotic pathways. Bone. 2007;40(2):345-353.

87. Kalu DN. The ovariectomized rat model of postmenopausal bone loss. Bone Miner. 1991;15(3):175-191.

88. Wronski TJ, Dann LM, Scott KS, Cintron M. Long-term effects of ovariectomy and aging on the rat skeleton. Calcif Tissue Int. 1989;45(6):360-366.

89. Lelovas PP, Xanthos TT, Thoma SE, Lyritis GP, Dontas IA. The laboratory rat as an animal model for osteoporosis research. Comp Med. 2008;58(5):424-430.

90. Jee WS, Yao W. Overview: Animal models of osteopenia and osteoporosis. J Musculoskelet Neuronal Interact. 2001;1(3):193-207. 
91. Thirunavukkarasu V, Nandhini AT, Anuradha CV. Lipoic acid prevents collagen abnormalities in tail tendon of high-fructose-fed rats. Diabetes Obes Metab. 2005;7(3):294-297.

92. Nandhini TA, Thirunavukkarasu V, Ravichandran MK, Anuradha CV. Taurine prevents fructose-diet induced collagen abnormalities in rat skin. J Diabetes Complications. 2005;19(5):305-311.

93. Wang X, Jia X, Chang T, Desai K, Wu L. Attenuation of hypertension development by scavenging methylglyoxal in fructose-treated rats. J Hypertens. 2008;26(4):765-772.

94. Kelley GL, Allan G, Azhar S. High dietary fructose induces a hepatic stress response resulting in cholesterol and lipid dysregulation. Endocrinology. 2004;145(2):548-555.

95. Reddy SS, Ramatholisamma P, Karuna R, Saralakumari D. Preventive effect of tinospora cordifolia against high-fructose diet-induced insulin resistance and oxidative stress in male wistar rats. Food Chem Toxicol. 2009;47(9):2224-2229.

96. Chace KV, Carubelli R, Nordquist RE. The role of nonenzymatic glycosylation, transition metals, and free radicals in the formation of collagen aggregates. Arch Biochem Biophys. 1991;288(2):473-480.

97. Lirio LM, Forechi L, Zanardo TC, et al. Chronic fructose intake accelerates non-alcoholic fatty liver disease in the presence of essential hypertension. J Diabetes Complications. 2015.

98. Tran LT, Yuen VG, McNeill JH. The fructose-fed rat: A review on the mechanisms of fructose-induced insulin resistance and hypertension. Mol Cell Biochem. 2009;332(1-2):145-159. 
99. Ackerman Z, Oron-Herman M, Grozovski M, et al. Fructose-induced fatty liver disease:

Hepatic effects of blood pressure and plasma triglyceride reduction. Hypertension.

2005;45(5):1012-1018.

100. Castro GS, Cardoso JF, Vannucchi H, Zucoloto S, Jordao AA. Fructose and NAFLD:

Metabolic implications and models of induction in rats. Acta Cir Bras. 2011;26 Suppl 2:45-50.

101. Heaney RP. Is there a role for bone quality in fragility fractures? Calcif Tissue Int. 1993;53

Suppl 1:S3-5; discussion S5-6.

102. Licata A. Bone density vs bone quality: What's a clinician to do? Cleve Clin J Med. 2009;76(6):331-336.

103. Marshall D, Johnell O, Wedel H. Meta-analysis of how well measures of bone mineral density predict occurrence of osteoporotic fractures. BMJ. 1996;312(7041):1254-1259.

104. Johnell O, Kanis JA, Oden A, et al. Predictive value of BMD for hip and other fractures. $J$ Bone Miner Res. 2005;20(7):1185-1194.

105. Adams JE. Single and dual energy X-ray absorptiometry. Eur Radiol. 1997;7 Suppl 2:S2031.

106. Bouxsein ML, Boyd SK, Christiansen BA, Guldberg RE, Jepsen KJ, Muller R. Guidelines for assessment of bone microstructure in rodents using micro-computed tomography. $J$ Bone Miner Res. 2010;25(7):1468-1486.

107. Bouxsein ML, Karasik D. Bone geometry and skeletal fragility. Curr Osteoporos Rep. 2006;4(2):49-56. 
108. Cole JH, van der Meulen MC. Whole bone mechanics and bone quality. Clin Orthop Relat Res. 2011;469(8):2139-2149.

109. Legrand E, Chappard D, Pascaretti C, et al. Trabecular bone microarchitecture, bone mineral density, and vertebral fractures in male osteoporosis. J Bone Miner Res. 2000;15(1):13-19.

110. Villanueva AR, Mehr LA. Modifications of the goldner and gomori one-step trichrome stains for plastic-embedded thin sections of bone. Am J Med Technol. 1977;43(6):536-538.

111. Turner RT. Cancellous bone turnover in growing rats: Time-dependent changes in association between calcein label and osteoblasts. J Bone Miner Res. 1994;9(9):1419-1424.

112. Dempster DW, Compston JE, Drezner MK, et al. Standardized nomenclature, symbols, and units for bone histomorphometry: A 2012 update of the report of the ASBMR histomorphometry nomenclature committee. J Bone Miner Res. 2013;28(1):2-17.

113. Ashraf JM, Ahmad S, Choi I, et al. Recent advances in detection of AGEs: Immunochemical, bioanalytical and biochemical approaches. IUBMB Life. 2015.

114. Hajek Z, Germanova A, Koucky M, et al. Detection of feto-maternal infection/inflammation by the soluble receptor for advanced glycation end products (sRAGE): Results of a pilot study. $J$ Perinat Med. 2008;36(5):399-404.

115. Wehr NB, Levine RL. Quantification of protein carbonylation. Methods Mol Biol. 2013;965:265-281 .

116. Dalle-Donne I, Aldini G, Carini M, Colombo R, Rossi R, Milzani A. Protein carbonylation, cellular dysfunction, and disease progression. J Cell Mol Med. 2006;10(2):389-406. 
117. Saito M, Marumo K. Collagen cross-links as a determinant of bone quality: A possible explanation for bone fragility in aging, osteoporosis, and diabetes mellitus. Osteoporos Int. 2010;21(2):195-214

118. Knott L, Bailey AJ. Collagen cross-links in mineralizing tissues: A review of their chemistry, function, and clinical relevance. Bone. 1998;22(3):181-187.

119. Gruber HE. Adaptations of goldner's masson trichrome stain for the study of undecalcified plastic embedded bone. Biotech Histochem. 1992;67(1):30-34.

120. Wronski TJ, Lowry PL, Walsh CC, Ignaszewski LA. Skeletal alterations in ovariectomized rats. Calcif Tissue Int. 1985;37(3):324-328.

121. Wronski TJ, Walsh CC, Ignaszewski LA. Histologic evidence for osteopenia and increased bone turnover in ovariectomized rats. Bone. 1986;7(2):119-123.

122. Parfitt AM. Bone histomorphometry: Proposed system for standardization of nomenclature, symbols, and units. Calcif Tissue Int. 1988;42(5):284-286.

123. Erben R. $<b r />$ Handbook of histology methods for bone and cartilage Totowna, New Jersey: Humana Press.

124. Lee TC, Mohsin S, Taylor D, et al. Detecting microdamage in bone. J Anat. 2003;203(2):161-172.

125. Parfitt AM, Drezner MK, Glorieux FH, et al. Bone histomorphometry: Standardization of nomenclature, symbols, and units. report of the ASBMR histomorphometry nomenclature committee. J Bone Miner Res. 1987;2(6):595-610. 
126. Angel NZ, Walsh N, Forwood MR, Ostrowski MC, Cassady AI, Hume DA. Transgenic mice overexpressing tartrate-resistant acid phosphatase exhibit an increased rate of bone turnover. $J$ Bone Miner Res. 2000;15(1):103-110.

127. de Castro UG, Dos Santos RA, Silva ME, de Lima WG, Campagnole-Santos MJ, Alzamora AC. Age-dependent effect of high-fructose and high-fat diets on lipid metabolism and lipid accumulation in liver and kidney of rats. Lipids Health Dis. 2013;12(1):136.

128. Kleiner DE, Brunt EM. Nonalcoholic fatty liver disease: Pathologic patterns and biopsy evaluation in clinical research. Semin Liver Dis. 2012;32(1):3-13.

129. Brunt EM, Tiniakos DG. Histopathology of nonalcoholic fatty liver disease. World $J$ Gastroenterol. 2010;16(42):5286-5296.

130. Willett TL, Sutty S, Gaspar A, Avery N, Grynpas M. In vitro non-enzymatic ribation reduces post-yield strain accommodation in cortical bone. Bone. 2013;52(2):611-622.

131. Bank RA, Beekman B, Verzij1 N, de Roos JA, Sakkee AN, TeKoppele JM. Sensitive fluorimetric quantitation of pyridinium and pentosidine crosslinks in biological samples in a single high-performance liquid chromatographic run. J Chromatogr B Biomed Sci Appl. 1997;703(1-2):37-44.

132. Bank RA, Jansen EJ, Beekman B, te Koppele JM. Amino acid analysis by reverse-phase high-performance liquid chromatography: Improved derivatization and detection conditions with 9-fluorenylmethyl chloroformate. Anal Biochem. 1996;240(2):167-176.

133. Hein G, Wiegand R, Lehmann G, Stein G, Franke S. Advanced glycation end-products pentosidine and $\mathrm{N}$ epsilon-carboxymethyllysine are elevated in serum of patients with osteoporosis. Rheumatology (Oxford). 2003;42(10):1242-1246. 
134. Nandhini TA, Thirunavukkarasu V, Ravichandran MK, Anuradha CV. Taurine prevents fructose-diet induced collagen abnormalities in rat skin. J Diabetes Complications. 2005;19(5):305-311.

135. Tsanzi E, Light HR, Tou JC. The effect of feeding different sugar-sweetened beverages to growing female sprague-dawley rats on bone mass and strength. Bone. 2008;42(5):960-968.

136. Schultz A, Neil D, Aguila MB, Mandarim-de-Lacerda CA. Hepatic adverse effects of fructose consumption independent of overweight/obesity. Int J Mol Sci. 2013;14(11):2187321886.

137. McElroy JF, Wade GN. Short- and long-term effects of ovariectomy on food intake, body weight, carcass composition, and brown adipose tissue in rats. Physiol Behav. 1987;39(3):361365.

138. Aitken JM, Armstrong E, Anderson JB. Osteoporosis after oophorectomy in the mature female rat and the effect of oestrogen and-or progestogen replacement therapy in its prevention. $J$ Endocrinol. 1972;55(1):79-87.

139. Jiang JM, Sacco SM, Ward WE. Ovariectomy-induced hyperphagia does not modulate bone mineral density or bone strength in rats. $J$ Nutr. 2008;138(11):2106-2110.

140. Rajasekar P, Anuradha CV. L-carnitine inhibits protein glycation in vitro and in vivo: Evidence for a role in diabetic management. Acta Diabetol. 2007;44(2):83-90.

141. Saito M, Fujii K, Soshi S, Tanaka T. Reductions in degree of mineralization and enzymatic collagen cross-links and increases in glycation-induced pentosidine in the femoral neck cortex in cases of femoral neck fracture. Osteoporos Int. 2006;17(7):986-995. 
142. Saito M, Marumo K, Kida Y, et al. Changes in the contents of enzymatic immature, mature, and non-enzymatic senescent cross-links of collagen after once-weekly treatment with human parathyroid hormone (1-34) for 18 months contribute to improvement of bone strength in ovariectomized monkeys. Osteoporos Int. 2011;22(8):2373-2383.

143. Black D, Farquharson C, Robins SP. Excretion of pyridinium cross-links of collagen in ovariectomized rats as urinary markers for increased bone resorption. Calcif Tissue Int. 1989;44(5):343-347.

144. Bass EF, Baile CA, Lewis RD, Giraudo SQ. Bone quality and strength are greater in growing male rats fed fructose compared with glucose. Nutr Res. 2013;33(12):1063-1071.

145. Turner RT, Vandersteenhoven JJ, Bell NH. The effects of ovariectomy and 17 beta-estradiol on cortical bone histomorphometry in growing rats. J Bone Miner Res. 1987;2(2):115-122.

146. Miller SC, Bowman BM, Miller MA, Bagi CM. Calcium absorption and osseous organ-, tissue-, and envelope-specific changes following ovariectomy in rats. Bone. 1991;12(6):439-446.

147. Parfitt AM, Mathews CH, Villanueva AR, Kleerekoper M, Frame B, Rao DS. Relationships between surface, volume, and thickness of iliac trabecular bone in aging and in osteoporosis. implications for the microanatomic and cellular mechanisms of bone loss. J Clin Invest. 1983;72(4):1396-1409.

148. Ke HZ, Crawford DT, Qi H, et al. A nonprostanoid EP4 receptor selective prostaglandin E2 agonist restores bone mass and strength in aged, ovariectomized rats. J Bone Miner Res. 2006;21(4):565-575. 
149. Hu S, Liu CC, Chen G, Willett T, Young RN, Grynpas MD. In vivo effects of two novel ALN-EP4a conjugate drugs on bone in the ovariectomized rat model for reversing postmenopausal bone loss. Osteoporos Int. 2015.

150. Parfitt AM. Caldwell, NJ: Telford Press; 1990.

151. Hanayama R, Shimizu H, Nakagami H, et al. Fluvastatin improves osteoporosis in fructosefed insulin resistant model rats through blockade of the classical mevalonate pathway and antioxidant action. Int J Mol Med. 2009;23(5):581-588.

152. Callaway DA, Jiang JX. Reactive oxygen species and oxidative stress in osteoclastogenesis, skeletal aging and bone diseases. J Bone Miner Metab. 2015;33(4):359-370.

153. Garrett IR, Boyce BF, Oreffo RO, Bonewald L, Poser J, Mundy GR. Oxygen-derived free radicals stimulate osteoclastic bone resorption in rodent bone in vitro and in vivo. J Clin Invest. 1990;85(3):632-639.

154. Yang S, Ries WL, Key LL,Jr. Nicotinamide adenine dinucleotide phosphate oxidase in the formation of superoxide in osteoclasts. Calcif Tissue Int. 1998;63(4):346-350.

155. Hall TJ, Schaeublin M, Jeker H, Fuller K, Chambers TJ. The role of reactive oxygen intermediates in osteoclastic bone resorption. Biochem Biophys Res Commun. 1995;207(1):280287.

156. Friess W. Collagen--biomaterial for drug delivery. Eur J Pharm Biopharm. 1998;45(2):113136.

157. Muthusami S, Ramachandran I, Muthusamy B, et al. Ovariectomy induces oxidative stress and impairs bone antioxidant system in adult rats. Clin Chim Acta. 2005;360(1-2):81-86. 
158. Lean JM, Davies JT, Fuller K, et al. A crucial role for thiol antioxidants in estrogendeficiency bone loss. J Clin Invest. 2003;112(6):915-923.

159. Sumiyoshi M, Satomi J, Kitazato KT, et al. PPARgamma-dependent and -independent inhibition of the HMGB1/TLR9 pathway by eicosapentaenoic acid attenuates ischemic brain damage in ovariectomized rats. J Stroke Cerebrovasc Dis. 2015;24(6):1187-1195.

160. Zhang R, Su D, Zhu W, et al. Estrogen suppresses adipogenesis by inhibiting S100A16 expression. J Mol Endocrinol. 2014;52(3):235-244.

161. Graham ML, Janecek JL, Kittredge JA, Hering BJ, Schuurman HJ. The streptozotocininduced diabetic nude mouse model: Differences between animals from different sources. Comp Med. 2011;61(4):356-360.

162. Candido R, Forbes JM, Thomas MC, et al. A breaker of advanced glycation end products attenuates diabetes-induced myocardial structural changes. Circ Res. 2003;92(7):785-792.

163. Reaven P, Merat S, Casanada F, Sutphin M, Palinski W. Effect of streptozotocin-induced hyperglycemia on lipid profiles, formation of advanced glycation endproducts in lesions, and extent of atherosclerosis in LDL receptor-deficient mice. Arterioscler Thromb Vasc Biol. 1997;17(10):2250-2256.

164. Civelek S, Gelisgen R, Andican G, et al. Advanced glycation end products and antioxidant status in nondiabetic and streptozotocin induced diabetic rats: Effects of copper treatment. Biometals. 2010;23(1):43-49.

165. Park L, Raman KG, Lee KJ, et al. Suppression of accelerated diabetic atherosclerosis by the soluble receptor for advanced glycation endproducts. Nat Med. 1998;4(9):1025-1031. 
166. Song X, Bao M, Li D, Li YM. Advanced glycation in D-galactose induced mouse aging model. Mech Ageing Dev. 1999;108(3):239-251. 